\title{
Finitary treatment of operator controlled derivations
}

\author{
Wilfried Buchholz (Universität München) \\ email: buchholz@rz.mathematik.uni-muenchen.de
}

May 3, 1999

\section{Introduction}

In this paper we continue our work on finitary representations of infinitary derivations which we have started in [Bu91] and resumed in [Bu97]. On the basis of [Bu92] we develop a finitary ordinal analysis of the axiom system KPi of Kripke-Platek set theory with an inaccessible universe. As a main result we obtain the following theorem: If $\mathrm{KPi} \vdash \forall z(" z=H F$ " $\rightarrow \forall x \in z \exists y \in z \phi(x, y))$ with $\phi(x, y) \in \Delta_{0}$, then there exists a $<\cdot$-primitive recursive function $F: \omega \rightarrow \omega$ (in the sense of [Tak87], p.117) with $\|<\cdot\|<\psi_{\Omega}\left(\varepsilon_{I+1}\right)$ such that $\forall n \in \omega \forall x \in L_{n} \exists y \in L_{F(n)} \phi(x, y)$ holds. Here " $z=\mathrm{HF}$ " is a set-theoretic formula saying " $z$ is the set of all hereditarily finite sets".

One goal of this line of work (from which we still are very far away) is to develop some kind of "semantics" for Arai's purely combinatorial proof-theoretic analysis of very strong impredicative theories (cf. [Ar96], [Ar97a], [Ar97b]), and to find connections to Rathjen's work [Ra91], [Ra94], [Ra95].

In $\S 1$ we introduce the language of $\mathcal{L}_{\mathrm{RS}}$ of ramified set theory and the infinitary proof system $\mathrm{RS}^{\infty}$, essentially as in [Bu92]. One technical modification is the following:

For RS-terms $a, b$ with $\operatorname{lev}(a)<\operatorname{lev}(b)$ we set: $a \stackrel{\circ}{\in} b: \equiv \begin{cases}B(a) & \text { if } b \equiv\left[x \in \mathrm{L}_{\beta}: B(x)\right] \\ \top & \text { if } b \equiv \mathrm{L}_{\beta}\end{cases}$

where $T$ is not considered as a formula, but we define $\top \wedge A: \equiv A$. This has the advantage that now $\exists x \in \mathrm{L}_{\beta} A(x) \simeq \bigvee(A(t))_{t \in \mathcal{T}_{\beta}}$ while in [Bu92] we had $\exists x \in \mathrm{L}_{\beta} A(x) \simeq \bigvee\left(t \notin \mathrm{L}_{0} \wedge A(t)\right)_{t \in \mathcal{T}_{\beta}}$.

At the end of $\S 1$ we introduce the main concept of this paper namely the notion of a notation system for $\mathrm{RS}^{\infty}$-derivations, and we define what it means that such a notation system is controlled by an operator $\mathcal{H}$.

In $\S 2$ we introduce a notation system $\mathbf{R S}^{0}$ which besides others contains notations for $\mathrm{RS}^{\infty}$-derivations of all KPi-axioms (relativized to $L_{I}$ ). In $\S 3$ we extend $\mathbf{R S}^{0}$ to a notation system $\mathbf{R S}^{+}$which in addition contains notations for $\mathrm{RS}^{\infty}$-derivations of all sequents $\phi_{1}^{\mathrm{L}_{I}}, \ldots, \phi_{n}^{\mathrm{L}_{I}}$ where $\phi_{1} \vee \ldots \vee \phi_{n}$ is a logically valid $\mathcal{L}_{\text {Ad }^{-}}$ formula. In $\S 4$ we introduce the collapsing functions $\psi_{\kappa}: O n \rightarrow \kappa$ and the operators $\mathcal{H}_{\gamma}: \mathcal{P}(O n) \rightarrow \mathcal{P}(O n)$ essentially as in [Bu92] but with the difference that in the present paper the so-called normalform condition " $\xi \in C\left(\xi, \psi_{\pi} \xi\right)$ " is integrated in the definition of $\psi_{\kappa}$. This facilitates the development of a primitive recursive ordinal notation system on the basis of the $\psi_{\kappa}$ 's. The details of such a development can be found for example in [Jä84] or [Bu86]. We also show how the relation " $\alpha \in \mathcal{H}_{\gamma}(X)$ " (for a finite set $X$ of ordinal notations) can characterized primitive recursively. In $\S 5$ we introduce a family $\left(\mathbf{H}_{\gamma}\right)_{\gamma \in O n}$ of notation systems for $\mathrm{RS}^{\infty}{ }_{-}$ derivations extending $\mathbf{R S}^{+} . \mathbf{H}_{\gamma}$ contains notations for $\mathrm{RS}^{\infty}$-derivations which are $\mathcal{H}_{\gamma}$-controlled and arise from $\mathrm{RS}^{\infty}$-derivations representable in $\mathbf{R S}^{+}$by application of the cut-elimination and collapsing procedures described in (the proofs of $3.14,3.16,3.17,4.8$ of) [Bu92]. $\S 6$ is concerned with the last part of the proof of the above stated theorem. We define a function $(h, n) \mapsto f(h, n)$ such that if $h \in \mathbf{H}_{\delta}$ denotes a cutfree $\mathrm{RS}^{\infty}$ derivation of $\forall x \in \mathrm{L}_{\omega} \exists y \in \mathrm{L}_{\omega} \phi(x, y)$ then $\forall n \in \omega \forall x \in L_{n} \exists y \in L_{f(h, n)} \phi(x, y)$ holds. The function $f$ is defined by recursion on the ordinal o $(h)$ of $h$, and a routine consideration shows that (under a canonical arithmetization) the function $n \mapsto f(h, n)$ for fixed $h$ is $<\cdot$-primitive recursive with a primitive recursive well ordering $<\cdot$ of order type $\|<\cdot\|=o(h)<\psi_{\Omega}\left(\varepsilon_{I+1}\right)$.

Remark: Two recent papers related to the present one are [Bla97] and [Tu98]. 


\section{$\S 1$ The language $\mathcal{L}_{R S}$ of ramified set theory and the infinitary proof system $\mathrm{RS}^{\infty}$}

Let $\mathcal{L}$ denote the usual first order language of set theory whose only nonlogical symbol is the binary predicate constant $\in$. The language $\mathcal{L}_{\text {Ad }}$ is obtained from $\mathcal{L}$ by adding the unary predicate constant Ad. The language $\mathcal{L}_{R S}$ of ramified set theory is obtained from $\mathcal{L}_{\text {Ad }}$ by adding a certain class $\mathcal{T}$ of individual constants, the so-called set terms or $R S$-terms. The definition of $\mathcal{T}$ will be given below. Before that we introduce some technical notions and abbreviations. In this context we use the letters $u, v$ to denote both, individual variables and RS-terms. Individual variables are indicated by $w, x, y, z$.

The formulas of $\mathcal{L}_{\mathrm{Ad}}$ are generated from literals $x \in y, \operatorname{Ad}(x), \neg(x \in y), \neg \operatorname{Ad}(x)$ by means of $\wedge, \vee, \forall, \exists$.

\section{Inductive definition of RS-formulas}

1. $u \in v, \neg(u \in v), \operatorname{Ad}(u), \neg \operatorname{Ad}(u)$ are RS-formulas (literals).

2. If $A, B$ are RS-formulas then so are $A \wedge B$ and $A \vee B$.

3. If $A$ is an RS-formula and $x \notin \mathrm{FV}(u)$ (which means that $u$ is not the variable $x$ ), then $\forall x(x \in u \rightarrow A)$ and $\exists x(x \in u \wedge A)$ are RS-formulas.

The negation $\neg A$ of an RS- or $\mathcal{L}_{\text {Ad }}$-formula $A$ is defined via de Morgan's laws.

$\Delta_{0}:=\left\{\phi: \phi\right.$ is an RS-formula of the language $\left.\mathcal{L}_{\mathrm{Ad}}\right\}=\{\phi: \phi$ is an RS-formula containing no RS-terms $\}$ Syntactic variables:

$A, B, C, D, F$ for RS-formulas, $\phi, \psi, \chi$ for $\mathcal{L}_{\text {Ad }}$-formulas, $u, v$ for elements of $\mathcal{T} \cup \operatorname{Var}$.

Finite sequences of variables are abbreviated by $\vec{x}, \vec{y}, \ldots$. The set of free (bound, resp.) variables of $A$ is denoted by $\mathrm{FV}(A)(\mathrm{BV}(A)$, resp.). If $\mathrm{FV}(A)=\emptyset$ then $A$ is called an $R S$-sentence.

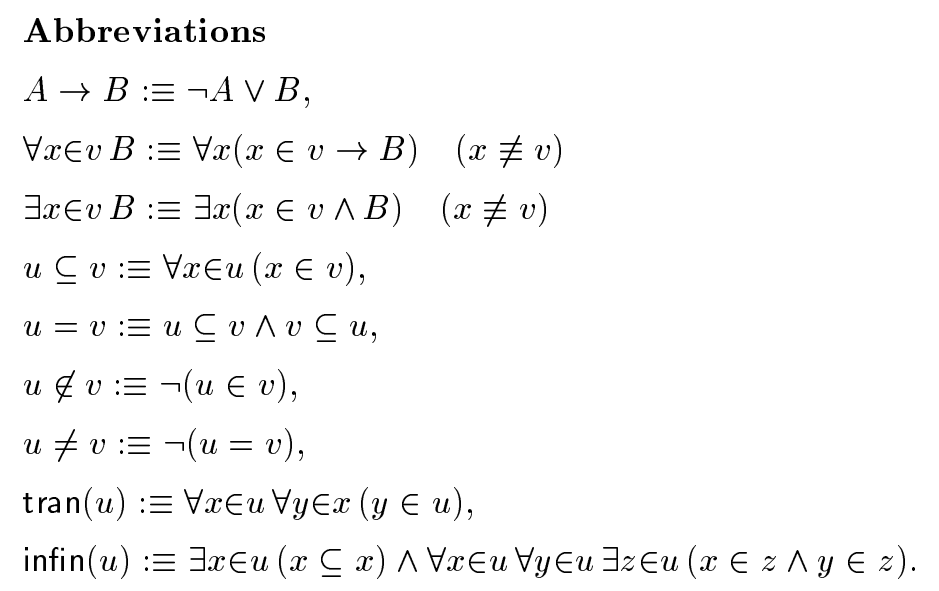

\section{Notation}

$[u \neq v]$ denotes $u \neq v$ or $v \neq u$. (Example: By $\Sigma \vdash[u \neq v], \neg A(u), A(v)$ we express that $\Sigma$ derives the sequents $u \neq v, \neg A(u), A(v)$ and $v \neq u, \neg A(u), A(v)$.) 
Definition of $\phi^{u}$ (w.l.o.g. $\left.u \notin \mathrm{BV}(\phi)\right)$

1. $\phi^{u}: \equiv \phi$ if $\phi$ is a literal,

2. $(\phi \wedge \psi)^{u}: \equiv \phi^{u} \wedge \psi^{u}$,

3. $(\forall x \in v \phi)^{u}: \equiv \forall x \in v \phi^{u},(\exists x \in v \phi)^{u}: \equiv \exists x \in v \phi^{u}$,

4. $(\forall x \phi)^{u}: \equiv \forall x \in u \phi^{u}$, if $\phi$ is not of the form $x \in v \rightarrow \psi$, $(\exists x \phi)^{u}: \equiv \exists x \in u \phi^{u}$, if $\phi$ is not of the form $x \in v \wedge \psi$.

Definition (RS-terms and their levels)

1. For every ordinal $\alpha$ the constant $\mathrm{L}_{\alpha}$ is an RS-term of level $\alpha$.

2. If $\phi$ is an $\mathcal{L}_{\text {Ad }}$-formula with $x \in \mathrm{FV}(\phi) \subseteq\left\{x, y_{1}, \ldots, y_{n}\right\}$, and if $a_{1}, \ldots, a_{n}$ are RS-terms of levels $<\alpha$ (where $\alpha>0$ ), then

$$
\left[x \in \mathrm{L}_{\alpha}: \phi^{\mathrm{L}_{\alpha}}\left(x, a_{1}, \ldots, a_{n}\right)\right]
$$

is an $R S$-term of level $\alpha$.

We denote the class of all RS-terms by $\mathcal{T}$, and the class of all RS-terms of level less than $\alpha$ by $\mathcal{T}_{\alpha}$.

In the following RS-terms are denoted by the letters $a, b, c, s, t$.

Note that an RS-term has no free variables.

Definition (Definition of $\mathrm{k}(\theta)$ and $\operatorname{lev}(\theta)$ )

If $\theta$ is an RS-term or RS-formula we set

$\mathrm{k}(\theta):=\left\{\alpha \in O n: \mathrm{L}_{\alpha}\right.$ occurs in $\left.\theta\right\}$ and $\operatorname{lev}(\theta):=\max (\mathrm{k}(\theta) \cup\{0\})$.

Here all occurrences of $\mathrm{L}_{\alpha}$, i.e. also those inside of subterms of $\theta$ are counted.

For technical reasons we also define $\mathrm{k}(0):=\mathrm{k}(1):=\emptyset, \quad \operatorname{lev}(0):=\operatorname{lev}(1):=0$.

\section{Remark}

For each $t \in \mathcal{T}$ we have level of $t=\operatorname{lev}(t)$. Hence $\mathcal{T}_{\alpha}=\{t \in \mathcal{T}: \operatorname{lev}(t)<\alpha\}$.

Abbreviation: $\mathcal{T}_{a}:=\mathcal{T}_{\operatorname{lev}(a)}, \quad \mathrm{k}(\theta)<\alpha: \Leftrightarrow \forall \xi \in \mathrm{k}(\theta)(\xi<\alpha)$

\section{Definition}

For RS-terms $a, b$ with $\operatorname{lev}(a)<\operatorname{lev}(b)$ we set:

$a \stackrel{\circ}{\in} b: \equiv\left\{\begin{array}{ll}B(a) & \text { if } b \equiv\left[x \in \mathrm{L}_{\beta}: B(x)\right] \\ \top & \text { if } b \equiv \mathrm{L}_{\beta}\end{array}\right.$ and $\quad a \stackrel{\notin}{\notin} b: \equiv \neg(a \stackrel{\circ}{\in} b)$, where $\neg \top: \equiv \perp$

$\top, \perp$ are not RS-formulas! We define $\top \rightarrow A: \equiv \top \wedge A: \equiv \perp \vee A: \equiv A$.

\section{Definition}

$\Omega_{0}:=0, \Omega_{\sigma}:=\aleph_{\sigma}$ for $\sigma>0$. Abbreviation: $\Omega:=\Omega_{1}$.

We assume the existence of a weakly inaccessible cardinal, i.e. a regular fixpoint of $\sigma \mapsto \Omega_{\sigma}$, and set $I:=\min \left\{\kappa: \kappa\right.$ regular \& $\left.\Omega_{\kappa}=\kappa\right\}, \quad \mathrm{R}:=\{\kappa: \omega<\kappa \leq I \& \kappa$ regular $\}=\{I\} \cup\left\{\Omega_{\sigma+1}: \sigma<I\right\}$.

For each ordinal $\alpha$ we set $\alpha^{+}:=\Omega_{\sigma+1}$ where $\sigma$ is determined by $\Omega_{\sigma} \leq \alpha<\Omega_{\sigma+1}$

In the following $\kappa, \pi, \tau$ always denote elements of $\mathrm{R}$. 


\section{Definition}

To each RS-sentence $A$ we assign a certain (possibly infinitary) conjunction $\bigwedge\left(A_{\iota}\right)_{\iota \in J}$ or disjunction $\bigvee\left(A_{\iota}\right)_{\iota \in J}$ of RS-sentences and we indicate this assignment by writing $A \simeq \bigwedge\left(A_{\iota}\right)_{\iota \in J}, A \simeq \bigvee\left(A_{\iota}\right)_{\iota \in J}$, resp.

1. $\operatorname{Ad}(a): \simeq \bigvee(t=a)_{t \in J}, \quad \neg \operatorname{Ad}(a): \simeq \bigwedge(t \neq a)_{t \in J} \quad$ with $J:=\left\{\mathrm{L}_{\kappa}: \kappa \in \mathrm{R} \& \kappa \leq \operatorname{lev}(a)\right\}$

2. $a \in b: \simeq \bigvee(t \stackrel{\circ}{\in} b \wedge t=a)_{t \in \mathcal{T}_{b}}, \quad a \notin b: \simeq \wedge(t \stackrel{\circ}{\in} b \rightarrow t \neq a)_{t \in \mathcal{T}_{b}}$

3. $\exists x \in b A(x): \simeq \bigvee(t \stackrel{\circ}{\in} b \wedge A(t))_{t \in \mathcal{T}_{b}}, \quad \forall x \in b A(x): \simeq \bigwedge(t \stackrel{\circ}{\in} b \rightarrow A(t))_{t \in \mathcal{T}_{b}}$

4. $\left(A_{0} \vee A_{1}\right): \simeq \bigvee\left(A_{\iota}\right)_{\iota \in\{0,1\}}, \quad\left(A_{0} \wedge A_{1}\right): \simeq \bigwedge\left(A_{\iota}\right)_{\iota \in\{0,1\}}$

We now define a rank-function for RS-sentences in such a way that $\forall \iota \in J\left(\operatorname{rk}\left(A_{\iota}\right)<\operatorname{rk}(A)\right)$ whenever $A \simeq \hat{\vee}\left(A_{\iota}\right)_{\iota \in J}$.

Definition (the rank of RS-sentences and RS-terms)

The $\operatorname{rank} \operatorname{rk}(\theta)$ of an RS-sentence or RS-term $\theta$ is defined by recursion on the number of symbols occurring in $\theta$ as follows:

1. $\operatorname{rk}\left(\mathrm{L}_{\alpha}\right):=\omega \cdot \alpha$

2. $\operatorname{rk}\left(\left[x \in \mathrm{L}_{\alpha}: A(x)\right]\right):=\max \left\{\omega \cdot \alpha+1, \operatorname{rk}\left(A\left(\mathrm{~L}_{0}\right)\right)+2\right\}$

3. $\operatorname{rk}(\operatorname{Ad}(a)):=\operatorname{rk}(\neg \operatorname{Ad}(a)):=\operatorname{rk}(a)+5$

4. $\operatorname{rk}(a \in b):=\operatorname{rk}(a \notin b):=\max \{\operatorname{rk}(a)+6, \operatorname{rk}(b)+1\}$

5. $\operatorname{rk}(\exists x \in b A(x)):=\operatorname{rk}(\forall x \in b A(x)):=\max \left\{\operatorname{rk}(b), \operatorname{rk}\left(A\left(\mathrm{~L}_{0}\right)\right)+2\right\}$

6. $\operatorname{rk}(A \wedge B):=\operatorname{rk}(A \vee B):=\max \{\operatorname{rk}(A), \operatorname{rk}(B)\}+1$

\section{Lemma 1.1}

Let $A \simeq \bigvee\left(A_{\iota}\right)_{\iota \in J}$ or $A \simeq \bigwedge\left(A_{\iota}\right)_{\iota \in J}$. Then the following holds.

a) $\operatorname{rk}(A)=\omega \cdot \operatorname{lev}(A)+n$, for some $n \in \omega$

b) $\operatorname{rk}\left(A_{\iota}\right)<\operatorname{rk}(A)$, for all $\iota \in J$

c) $\mathrm{k}\left(A_{\iota}\right) \subseteq \mathrm{k}(A) \cup \mathrm{k}(\iota)$, for all $\iota \in J$

d) $\operatorname{rk}(A)=\omega \cdot \alpha \Longrightarrow A \equiv \exists x \in \mathrm{L}_{\alpha} B(x)$ or $A \equiv \forall x \in \mathrm{L}_{\alpha} B(x)$

e) $\operatorname{rk}(A)=\operatorname{rk}(\neg A)$

Proof: cf. [Bu92]

\section{Inductive Definition of the sets $\Sigma(\kappa)$ of RS-sentences}

1. If $A$ is an RS-sentence with $\mathrm{k}(A) \subseteq \kappa$, then $A \in \Sigma(\kappa)$.

2. If $A, B \in \Sigma(\kappa)$, then $A \wedge B \in \Sigma(\kappa)$ and $A \vee B \in \Sigma(\kappa)$.

3. If $A \equiv Q x \in a B(x)$ with $B\left(\mathrm{~L}_{0}\right) \in \Sigma(\kappa)$ and $\operatorname{lev}(a)<\kappa$, then $A \in \Sigma(\kappa)$.

4. If $A \equiv \exists x \in \mathrm{L}_{\kappa} B(x)$ with $B\left(\mathrm{~L}_{0}\right) \in \Sigma(\kappa)$, then $A \in \Sigma(\kappa)$.

$\Pi(\kappa):=\{\neg A: A \in \Sigma(\kappa)\}$

Remark: $\Sigma(\kappa) \cap \Pi(\kappa)=\{A: \mathrm{FV}(A)=\emptyset \& \mathbf{k}(A) \subseteq \kappa\}$ 
Definition of $A^{(u, \kappa)}$ (w.l.o.g. $u \notin \mathrm{BV}(A)$ )

1. $A^{(u, \kappa)}: \equiv A$ if $A$ is a literal,

2. $(A \diamond B)^{(u, \kappa)}: \equiv A^{(u, \kappa)} \diamond B^{(u, \kappa)}$,

3. $(Q x \in v A)^{(u, \kappa)}: \equiv Q x \in v A^{(u, \kappa)}$, if $v \not \equiv \mathrm{L}_{\kappa}$,

4. $\left(Q x \in \mathrm{L}_{\kappa} A\right)^{(u, \kappa)}: \equiv Q x \in u A^{(u, \kappa)}$.

Definition $A^{(\beta, \kappa)}:= \begin{cases}A^{\left(\mathrm{L}_{\beta}, \kappa\right)} & \text { if } \beta<\kappa \\ A & \text { otherwise }\end{cases}$

\section{Inferences and derivations}

Finite sets of RS-formulas are called sequents.

Syntactic variables for sequents are $\Gamma, \Gamma^{\prime}, \Delta$.

In the following we mostly write $A_{1}, \ldots, A_{n}$ for $\left\{A_{1}, \ldots, A_{n}\right\}$, and $A, \Gamma, \Gamma^{\prime}$ for $\{A\} \cup \Gamma \cup \Gamma^{\prime}$, etc.

$\mathrm{k}(\Gamma):=\bigcup_{A \in \Gamma} \mathrm{k}(A)$

In the sequel certain formal expressions $\mathcal{I}$ will be declared as inferences (or inference symbols).

For each inference $\mathcal{I}$ the following data will be defined

- a set $|\mathcal{I}|$ (the arity of $\mathcal{I}$ ),

- a sequent $\Delta(\mathcal{I})$ (the elements of $\Delta(\mathcal{I})$ are called principal formulas of $\mathcal{I}$ ),

- a family of sequents $\left(\Delta_{\iota}(\mathcal{I})\right)_{\iota \in|\mathcal{I}|}\left(\right.$ the elements of $\Delta_{\iota}(\mathcal{I})$ are called minor formulas of $\left.\mathcal{I}\right)$,

- a set $\operatorname{Eig}(\mathcal{I})$ which is either empty or a singleton $\{y\}$ with $y \notin \mathrm{FV}(\Delta(\mathcal{I}))$;

in the latter case $y$ is called the eigenvariable of $\mathcal{I}$,

- a finite set $\mathrm{k}(\mathcal{I}) \subseteq O n$.

\section{Inductive definition of derivations}

If $\mathcal{I}$ is an inference, and $\left(d_{\iota}\right)_{\iota \in|\mathcal{I}|}$ is a family of derivations such that $\operatorname{Eig}(\mathcal{I}) \cap \mathrm{FV}(\Gamma)=\emptyset$, and

$$
\Gamma:=\Delta(\mathcal{I}) \cup \bigcup_{\iota \in|\mathcal{I}|}\left(\operatorname{End}\left(d_{\iota}\right) \backslash \Delta_{\iota}(\mathcal{I})\right) \text { is finite }
$$

then $d:=\mathcal{I}\left(d_{\iota}\right)_{\iota \in|\mathcal{I}|}$ is a derivation with $\operatorname{End}(d):=\Gamma, \operatorname{depth}(d):=\sup \left\{\operatorname{depth}\left(d_{\iota}\right)+1: \iota \in|\mathcal{I}|\right\}$.

$\operatorname{End}(d)$ is called the endsequent of $d$.

An inference $\mathcal{I}$ is called finitary if $|\mathcal{I}|=\{0, \ldots, n-1\} \in \omega$.

A derivation $d$ is called finitary if all its inferences are finitary.

NOTATION

1. By writing $(\mathcal{I}) \frac{\ldots \Delta_{\iota} \ldots(\iota \in J)}{\Delta}[! y !]$ we declare $\mathcal{I}$ as an inference symbol with $|\mathcal{I}|=J, \Delta(\mathcal{I})=\Delta, \Delta_{\iota}(\mathcal{I})=\Delta_{\iota}$, and $\operatorname{Eig}(\mathcal{I})=\emptyset[\operatorname{Eig}(\mathcal{I})=\{y\}$, resp. $]$

2. If $|\mathcal{I}|=\{0, \ldots, n\}$ we write $(\mathcal{I}) \frac{\Delta_{0} \Delta_{1} \ldots \Delta_{n}}{\Delta}$ instead of $(\mathcal{I}) \frac{\ldots \Delta_{\iota} \ldots(\iota \in J)}{\Delta}$. 
If $|\mathcal{I}|=\emptyset$ we write $(\mathcal{I}) \Delta$ instead of $(\mathcal{I}) \frac{}{\Delta}$.

3. Inference symbols $\mathcal{I}$ with $|\mathcal{I}|=\emptyset$ will be called axioms or atomic derivations.

4. If $|\mathcal{I}|=\{0, \ldots, n-1\}$ we write $d=\mathcal{I} d_{0} \ldots d_{n-1}$ instead of $d=\mathcal{I}\left(d_{\iota}\right)_{\iota \in|\mathcal{I}|}$.

\section{Remark}

If $d=\mathcal{I}\left(d_{\iota}\right)_{\iota \in|\mathcal{I}|}$ is a derivation with $\Delta(\mathcal{I}) \subseteq \Gamma$ and $(\forall \iota \in|\mathcal{I}|) \operatorname{End}\left(d_{\iota}\right) \subseteq \Gamma \cup \Delta_{\iota}(\mathcal{I})$, then $\operatorname{End}(d) \subseteq \Gamma$.

\section{Definition}

For all finitary derivations $d$ we define $\mathrm{k}(d) \subseteq O n$ and $\mathrm{k}_{c}(d) \subseteq O n$ as follows:

$\mathrm{k}\left(\mathcal{I} d_{0} \ldots d_{n-1}\right):=\mathrm{k}(\mathcal{I}) \cup \bigcup_{i<n} \mathrm{k}\left(d_{i}\right), \quad \mathrm{k}_{c}\left(\mathcal{I} d_{0} \ldots d_{n-1}\right):=(\mathrm{k}(\mathcal{I}) \backslash \mathrm{k}(\Delta(\mathcal{I}))) \cup \bigcup_{i<n} \mathrm{k}_{c}\left(d_{i}\right)$.

\section{Lemma 1.2}

If $d$ is a finitary derivation such that $\forall i \in|\mathcal{I}|\left[\mathrm{k}\left(\Delta_{i}(\mathcal{I})\right) \subseteq \mathrm{k}(\mathcal{I}) \cup \mathrm{k}(\Delta(\mathcal{I}))\right]$ holds for each inference occurring in $d$, then $\mathrm{k}(d) \subseteq \mathrm{k}(\operatorname{End}(d)) \cup \mathrm{k}_{c}(d)$.

Proof:

Let $d=\mathcal{I} d_{0} \ldots d_{n-1}$. Then $\mathrm{k}(d)=\mathrm{k}(\mathcal{I}) \cup \bigcup_{i<n} \mathrm{k}\left(d_{i}\right) \stackrel{\mathrm{IH}}{\subseteq} \mathrm{k}(\mathcal{I}) \cup \bigcup_{i<n} \mathrm{k}\left(\operatorname{End}\left(d_{i}\right)\right) \cup \bigcup_{i<n} \mathrm{k}_{c}\left(d_{i}\right) \subseteq$ $\mathrm{k}(\Delta(\mathcal{I})) \cup \bigcup_{i<n} \mathrm{k}\left(\operatorname{End}\left(d_{i}\right) \backslash \Delta_{i}(\mathcal{I})\right) \cup(\mathrm{k}(\mathcal{I}) \backslash \mathrm{k}(\Delta(\mathcal{I}))) \cup \bigcup_{i<n} \mathrm{k}_{c}\left(d_{i}\right)=\mathrm{k}(\operatorname{End}(d)) \cup \mathrm{k}_{c}(d)$.

\section{Definition}

A proof system is a class of inference symbols.

A proof system $\mathfrak{S}$ is called finitary if all its inferences are finitary.

A derivation $d$ is called an $\mathfrak{S}$-derivation if all inferences occurring in $d$ belong to $\mathfrak{S}$.

The infinitary proof system $\mathrm{RS}^{\infty}$

formulas: RS-sentences

inferences:

$$
\begin{aligned}
& \left(\bigwedge_{A}\right) \frac{\ldots A_{\iota} \ldots(\iota \in J)}{A}, \text { if } A \simeq \bigwedge\left(A_{\iota}\right)_{\iota \in J} \\
& \left(\bigvee_{A}^{\iota_{0}}\right) \frac{A_{\iota_{0}}}{A}, \text { if } A \simeq \bigvee\left(A_{\iota}\right)_{\iota \in J} \text { and } \iota_{0} \in J \\
& \left(\operatorname{Cut}_{C}\right) \frac{C \quad \neg C}{\emptyset} \\
& \left(\operatorname{Ref}_{A}^{\kappa}\right) \frac{A}{\exists z \in \mathrm{L}_{\kappa} A^{(z, \kappa)}}, \text { if } A \in \Sigma(\kappa) \text { and } \kappa \in \mathrm{k}(A)
\end{aligned} \quad \mathrm{k}(\mathcal{I}):= \begin{cases}\mathrm{k}(C) & \text { if } \mathcal{I}=\mathrm{Cut}_{C} \\
\mathrm{k}(A) \cup \mathrm{k}\left(\iota_{0}\right) & \text { if } \mathcal{I}=\bigvee_{A}^{\iota_{0}} \\
\mathrm{k}(\Delta(\mathcal{I})) & \text { otherwise }\end{cases}
$$




\section{DEFINITION}

Assume that the following entities are given:

A nonempty set $\mathcal{D}$ of finitary derivations $d$ with $\mathrm{FV}(\operatorname{End}(d))=\emptyset$, and mappings

o $: \mathcal{D} \rightarrow O n$,

$\operatorname{deg}: \mathcal{D} \rightarrow$ On,

tp : $\mathcal{D} \rightarrow \mathrm{RS}^{\infty}$ (the class of $\mathrm{RS}^{\infty}$-inferences)

[] $:\{(d, \iota): d \in \mathcal{D} \& \iota \in|\operatorname{tp}(d)|\} \rightarrow \mathcal{D}$, we write $d[\iota]$ for []$(d, \iota)$.

Abbreviation: $\mathcal{D} \ni d \vdash_{\rho}^{\alpha} \Gamma: \Longleftrightarrow d \in \mathcal{D} \& \operatorname{End}(d) \subseteq \Gamma \& \circ(d)=\alpha \& \operatorname{deg}(d) \leq \rho$.

$(\mathcal{D}, \mathrm{o}, \mathrm{deg}, \mathrm{tp},[])$ is called a notation system for $\mathrm{RS}^{\infty}$-derivations if $\mathcal{D} \ni d \vdash_{\rho}^{\alpha} \Gamma$ implies:

(a) $\Delta(\operatorname{tp}(d)) \subseteq \Gamma$,

(b) $\mathcal{D} \ni d[\iota] \vdash_{\rho}^{\alpha_{\iota}} \Gamma, \Delta_{\iota}(\operatorname{tp}(d))$ with $\alpha_{\iota}<\alpha \quad(\iota \in|\operatorname{tp}(d)|)$,

(c) $\operatorname{tp}(d)=\mathrm{Cut}_{C} \Rightarrow \operatorname{rk}(C)<\rho$,

(d) $\operatorname{tp}(d)=\bigvee_{A}^{\iota_{0}} \Rightarrow \mathrm{k}\left(\iota_{0}\right)<\alpha$.

We say that the notation system $(\mathcal{D}, \mathrm{o}, \mathrm{deg}, \mathrm{tp},[])$ is controlled by the operator $\mathcal{H}: \mathcal{P}(O n) \rightarrow \mathcal{P}(O n)$ if for each $d \in \mathcal{D}$ the following holds

(e) $\mathrm{k}(\operatorname{tp}(d)) \cup\{\mathrm{o}(d)\} \subseteq \mathcal{H}(\mathrm{k}(d))$,

(f) $\mathrm{k}(d[\iota]) \subseteq \mathcal{H}(\mathrm{k}(d) \cup \mathrm{k}(\iota))$, for all $\iota \in|\operatorname{tp}(d)|$.

\section{Remark}

Let $(\mathcal{D}, \mathrm{o}, \mathrm{deg}, \mathrm{tp},[])$ be a notation system for $\mathrm{RS}^{\infty}$-derivations.

For each $d \in \mathcal{D}$ we define its interpretation $d^{\infty}$ by recursion on $\mathrm{o}(d): d^{\infty}:=\operatorname{tp}(d)\left(d[\iota]^{\infty}\right)_{\iota \in|\operatorname{tp}(d)|}$.

One easily verifies that $d^{\infty}$ is an $\operatorname{RS}^{\infty}$-derivation with

$\operatorname{End}\left(d^{\infty}\right) \subseteq \operatorname{End}(d), \operatorname{depth}\left(d^{\infty}\right) \leq \mathrm{o}(d)$, and $\operatorname{rk}(C)<\operatorname{deg}(d)$ for each Cut ${ }_{C}$ occurring in $d^{\infty}$.

\section{$\S 2$ The notation system $\mathbf{R S}^{0}$}

$\mathbf{R S}^{0}$ will contain notations for $\mathrm{RS}^{\infty}$-derivations of all KPi-axioms relativized to $L_{I}$.

The axiom system $\mathrm{KPi}$

(Ext) $\quad \forall x \forall y \forall z[x=y \rightarrow(x \in z \rightarrow y \in z)]$ and $\forall x \forall y[x=y \rightarrow(\operatorname{Ad}(x) \rightarrow \operatorname{Ad}(y))]$

(Found) $\forall \vec{z} \forall x_{0}\left[\forall x(\forall y \in x \phi(y, \vec{z}) \rightarrow \phi(x, \vec{z})) \rightarrow \phi\left(x_{0}, \vec{z}\right)\right]$

(Pair) $\quad \forall x \forall y \exists z(x \in z \wedge y \in z)$

(Union) $\forall x \exists z \forall y \in x(y \subseteq z)$

$(\phi$-Sep $) \quad \forall \vec{z} \forall w \exists y[\forall x \in y(x \in w \wedge \phi(x, \vec{z})) \wedge \forall x \in w(\phi(x, \vec{z}) \rightarrow x \in y)] \quad\left(\phi \in \Delta_{0}\right)$

(Infin) $\quad \forall x \exists w \operatorname{infin}(w) \quad$ (Note that $\mathrm{FV}(\exists w \operatorname{infin}(w))=\emptyset$; the quantifier $\forall x$ has technical reasons.)

$(\phi$-Col $) \quad \forall \vec{z} \forall w\left[\forall x \in w \exists y \phi(x, y, \vec{z}) \rightarrow \exists w_{1} \forall x \in w \exists y \in w_{1} \phi(x, y, \vec{z})\right] \quad\left(\phi \in \Delta_{0}\right)$

(Ad1) $\quad \forall x[\operatorname{Ad}(x) \rightarrow \operatorname{tran}(x)]$ 
$(\operatorname{Ad} 2)$

Definition of $\mathcal{A} \mathcal{X}^{0}$

Let $\mathcal{A} \mathcal{X}^{0}$ be the set of all finite sequences of RS-sentences falling under one of the schemata (1)-(17).

(1) $\left(\forall x_{k} \phi\left(a_{0}, \ldots, a_{k-1}, x_{k}\right)\right)^{\lambda}$ if $a_{0}, \ldots, a_{k-1} \in \mathcal{T}_{\lambda}$, and $\forall x_{0} \ldots \forall x_{k} \phi\left(x_{0}, \ldots, x_{k}\right)$ is one of the KPi-axioms (Ext), (Found), (Pair), (Union), ( $\Delta_{0}$-Sep), (Ad1), (Ad2) with $\lambda \in \operatorname{Lim}$ or (Infin), $\left(\Delta_{0}\right.$-Col) with $\lambda \in \mathrm{R}$.

(2) $\forall x \in \mathrm{L}_{I} \exists y \in \mathrm{L}_{I}(\operatorname{Ad}(y) \wedge x \in y)$

(3) $a=a$,

(4) $a \subseteq a$,

(5) $b \subseteq \mathrm{L}_{\alpha}, \quad$ if $\operatorname{lev}(b) \leq \alpha$,

(6) $\forall x \in a\left(x \subseteq \mathrm{L}_{\alpha}\right), \quad$ if $\operatorname{lev}(a) \leq \alpha+1$,

(7) $\forall x \in b(x \in a \wedge F(x)), \quad$ if $b \equiv\left[x \in \mathrm{L}_{\beta}: x \in a \wedge F(x)\right]$,

(8) $\forall x \in a(F(x) \rightarrow x \in b)$, if $b \equiv\left[x \in \mathrm{L}_{\beta}: x \in a \wedge F(x)\right]$,

(9) $\neg \operatorname{Ad}(a), \operatorname{tran}(a), \operatorname{tran}(a)$,

(10) $\neg \operatorname{Ad}(a), \psi^{a}, \psi^{a}, \quad$ if $\psi$ is an axiom (Pair),(Union),(Infin), $\left(\Delta_{0}-\operatorname{Sep}\right),\left(\Delta_{0}\right.$-Col)

(11) $\exists x \in \mathrm{L}_{\alpha}(\forall y \in x A(y) \wedge \neg A(x)), \forall x \in a A(x), \quad$ if $\operatorname{lev}(a) \leq \alpha$,

(12) $\left[s_{1} \neq t_{1}\right], \ldots,\left[s_{n} \neq t_{n}\right], \neg A(\vec{s}), A(\vec{t}), \quad$ if in $A(\vec{x})$ each of the variables $\vec{x}$ occurs at most once.

(13) $\left[s_{1} \neq t_{1}\right], \ldots,\left[s_{n-1} \neq t_{n-1}\right], a \notin t_{n}, \neg B\left(s_{1}, \ldots, s_{n-1}, a\right), A\left(t_{1}, \ldots, t_{n}\right)$, if $A(\vec{x}) \equiv \exists y \in x_{n} B\left(x_{1}, \ldots, x_{n-1}, y\right)$, and in $A(\vec{x})$ each of the variables $\vec{x}$ occurs at most once.

(14) $\left[s_{1} \neq t_{1}\right], a \notin t_{2}, a \neq s_{1}, t_{1} \in t_{2}$,

(15) $\forall x \in \mathrm{L}_{\omega} \exists u \in \mathrm{L}_{\omega}(\exists y \in u(x \in y) \wedge \mathcal{A}(u))$ with $\mathcal{A}(u): \equiv \forall x \in u(\forall y \in x(y \neq y) \vee \mathcal{B}(u, x))$ and $\mathcal{B}(u, x): \equiv \exists x_{0} \in u\left(x_{0} \in x \wedge \forall y \in x\left(y \subseteq x_{0}\right)\right)$

(16) $\mathcal{A}\left(a_{n}\right)$ with $a_{n}:=\left[x \in \mathrm{L}_{n+1}: x=\mathrm{L}_{0} \vee \ldots \vee x=\mathrm{L}_{n}\right]$

(17) $\forall y \in \mathrm{L}_{0}(y \neq y)$.

\section{Remarks}

ad (12)-(14): Remember that $\left[s_{i} \neq t_{i}\right]$ denotes $s_{i} \neq t_{i}$ or $t_{i} \neq s_{i}$.

ad (12): If the number of free occurrences of $x$ in $A(x)$ is $\leq n$, then $(\underbrace{s \neq t, \ldots, s \neq t}_{n}, \neg A(s), A(t))$ is an instance of $(12)$.

ad (15),(16): Later we will use the formula " $v=\mathrm{HF}$ " $: \equiv \operatorname{tran}(v) \wedge \exists x \in v(x \subseteq x) \wedge(\text { Pair })^{v} \wedge(\text { Union })^{v} \wedge$ $(\phi \text {-Sep })^{v} \wedge \forall x \in v \exists u \in v(\exists y \in u(x \in y) \wedge \mathcal{A}(u))$ with $\phi\left(x, z_{1}, z_{2}\right): \equiv x \in z_{1} \vee x=z_{2}$ in order to express " $v$ is the set of all hereditarily finite sets".

Definition of $o(\Pi)$ for $\Pi \in \mathcal{A} \mathcal{X}^{0}$

Let $\Pi=\left(A_{1}, \ldots, A_{n}\right)$. $o(\Pi):= \begin{cases}\omega^{\mathrm{rk}\left(\varphi(\vec{a})^{\lambda}\right)} \# \omega \cdot \lambda & \text { if } \Pi=\left(\varphi(\vec{a})^{\lambda}\right) \text { is of kind (1)(Found) } \\ \omega^{\mathrm{rk}(\forall x \in a A(x))} \# \omega \cdot \operatorname{lev}(a) & \text { if } \Pi=(F, \forall x \in a A) \text { is of kind (11) } \\ \omega^{\mathrm{rk}\left(A_{1}\right)} \# \ldots \# \omega^{\mathrm{rk}\left(A_{n}\right)} & \text { otherwise }\end{cases}$ 
Definition of $\operatorname{deg}(\Pi)$ for $\Pi \in \mathcal{A} \mathcal{X}^{0}$

$\operatorname{deg}(\Pi):= \begin{cases}\omega \cdot \lambda & \text { if } \Pi=\left(\phi^{\lambda}\right) \text { is of kind }(1)(\operatorname{Ad} 1) \text { or }(1)(\operatorname{Ad} 2) \\ \omega \cdot(\operatorname{lev}(a)+1) & \text { if } \Pi=(\neg \operatorname{Ad}(a), C(a)) \text { is of kind }(9) \text { or }(10) \\ \omega^{2} & \text { if } \Pi \text { is of kind }(15) \text { or }(16) \\ 0 & \text { otherwise }\end{cases}$

\section{Definition}

For each $\Pi=\left(F_{1}, \ldots, F_{m}\right) \in \mathcal{A} \mathcal{X}^{0}$ of kind $(j)$, we introduce an inference $A x_{j}^{*} \Pi$ with $\left|A x_{j}^{*} \Pi\right|:=\emptyset, \Delta\left(\mathrm{Ax}_{j}^{*} \Pi\right):=\left\{F_{1}, \ldots, F_{m}\right\}, \mathrm{k}\left(\mathrm{Ax}{ }_{j}^{*} \Pi\right):=\mathrm{k}\left(\Delta\left(\mathrm{Ax}_{j}^{*} \Pi\right)\right)=\bigcup_{i=1}^{m} \mathrm{k}\left(F_{i}\right)$.

\section{Definition of the finitary proof system $\mathrm{RS}^{0}$}

formulas: RS-sentences (as for $\mathrm{RS}^{\infty}$ )

inferences: $\mathrm{Ax}_{j}^{*} \Pi$, and $\bigwedge_{A_{0} \wedge A_{1}}, \bigvee_{A}^{\iota_{0}}, \operatorname{Cut}_{C}, \operatorname{Ref}_{A}^{\kappa}\left(\right.$ as in $\left.\operatorname{RS}^{\infty}\right)$.

Definition of the notation system $\mathbf{R S}^{0}=\left(\mathcal{D}_{0}, o, \operatorname{deg}\right.$, tp, [] $)$

$\mathcal{D}_{0}:=$ set of all $\mathrm{RS}^{0}$-derivations

$\mathrm{o}\left(\mathcal{I} d_{0} \ldots d_{n-1}\right):= \begin{cases}\mathrm{o}(\Pi) & \text { if } \mathcal{I}=\mathrm{Ax}_{j}^{*} \Pi \\ \max \left\{\mathrm{o}\left(d_{0}\right), \operatorname{lev}\left(\iota_{0}\right)\right\}+1 & \text { if } \mathcal{I}=\bigvee_{A}^{\ell_{0}} \\ \max \left\{\mathrm{o}\left(d_{0}\right), \ldots, \mathrm{o}\left(d_{n-1}\right)\right\}+1 & \text { otherwise }\end{cases}$

$\operatorname{deg}\left(\mathcal{I} d_{0} \ldots d_{n-1}\right):= \begin{cases}\operatorname{deg}(\Pi) & \text { if } \mathcal{I}=\mathrm{Ax}_{j}^{*} \Pi \\ \max \left\{\operatorname{rk}(C)+1, \operatorname{deg}\left(d_{0}\right), \operatorname{deg}\left(d_{1}\right)\right\} & \text { if } \mathcal{I}=\mathrm{Cut}_{C} \\ \max \left\{\operatorname{deg}\left(d_{0}\right), \ldots, \operatorname{deg}\left(d_{n-1}\right)\right\} & \text { otherwise }\end{cases}$

Definition of $\operatorname{tp}(d)$ and $d[\iota]$

$\operatorname{tp}\left(\operatorname{Ax}_{j}^{*} \Pi\right):=\bigwedge_{A}$ where $A$ is the leftmost $\Lambda$-formula in $\Pi$,

e.g. $\operatorname{tp}\left(\mathrm{Ax}_{4}^{*}(a \subseteq a)\right)=\bigwedge_{a \subseteq a}, \operatorname{tp}\left(\mathrm{Ax}_{14}^{*}\left(s_{1} \neq t_{1}, a \notin t_{2}, a \neq s_{1}, t_{1} \in t_{2}\right)\right)=\bigwedge_{a \notin t_{2}}$

$\left(\mathrm{Ax}_{j}^{*} \Pi\right)[\iota]$ will be defined below!

If $d=\mathcal{I} d_{0} \ldots d_{n}\left(\right.$ with $\left.\mathcal{I} \neq \mathrm{A} x_{j}^{*} \Pi\right)$ then $\operatorname{tp}(d):=\mathcal{I}$ and $d[i]:=d_{i}$.

\section{Definition}

For $X \subseteq O n$ let $\mathcal{H}^{0}(X)$ be the closure of $X \cup\{0,1\}$ under \#, $\lambda x \cdot \omega \cdot x, \lambda x \cdot \omega^{x}$, and $\lambda x \cdot x^{+}$.

\section{Theorem 2.1}

$\mathbf{R S}^{0}$ is a notation system for $\mathrm{RS}^{\infty}$-derivations, and is controlled by $\mathcal{H}^{0}$.

Proof:

Assume $\mathcal{D}_{0} \ni d \vdash_{\rho}^{\alpha} \Gamma$. We have to prove propositions (a),..,(f) in definition of "notation system for $\mathrm{RS}^{\infty}{ }_{-}$ derivations" from $\S 2$. This is done by induction on the build up of $d$.

For $d=\mathcal{J} d_{0} \ldots d_{n}$ (with $\mathcal{J} \neq A x_{j}^{*} \Pi$ ) all propositions are trivial or immediate consequences of the IH.

It remains to prove (a),.., (f) for $d=\mathrm{Ax}_{j}^{*} \Pi$. Let $\Pi=\left(F_{1}, \ldots, F_{m}\right)$.

We have $\mathcal{I}:=\operatorname{tp}(d)=\bigwedge_{B}$ with $B \simeq \bigwedge\left(B_{\iota}\right)_{\iota \in J} \in\left\{F_{1}, \ldots, F_{m}\right\}$.

(a) $\Delta(\mathcal{I})=\{B\} \subseteq\left\{F_{1}, \ldots, F_{m}\right\}=\Delta\left(\mathrm{Ax}_{j}^{*} \Pi\right)=\operatorname{End}(d) \subseteq \Gamma$.

(b) $\mathcal{D}_{0} \ni d[\iota] \vdash_{\rho}^{\mathrm{o}(d[\iota])} \Gamma, \Delta_{\iota}(\mathcal{I})$ are easily verified by inspection of the below definition of $\left(\mathrm{Ax}_{j}^{*} \Pi\right)[\iota]$. 
For $\mathrm{o}(d[\iota])<\alpha$ we only have to check that $\mathrm{o}\left(\Pi^{\prime}\right)<\mathrm{o}(\Pi)$ for each axiom $\mathrm{Ax}_{j}^{*} \Pi^{\prime}$ occurring in $d[\iota]$, and that $\operatorname{lev}(t)<\mathrm{o}(\Pi)$ for each $\bigvee_{A}^{t}$ occurring in $d[\iota]$. Then $\mathrm{o}(d[\iota])<\mathrm{o}(\Pi)=\mathrm{o}(d) \leq \alpha$, since $\mathrm{o}(\Pi) \in \mathrm{Lim}$.

In almost all cases the relation $\mathrm{o}\left(\Pi^{\prime}\right), \operatorname{lev}(t)<\mathrm{o}(\Pi)$ follows immediately from

(*) $A \simeq \bigvee_{\iota \in J} A_{\iota}$ or $A \simeq \bigwedge_{\iota \in J} A_{\iota} \Longrightarrow \operatorname{rk}\left(A_{\iota}\right), \operatorname{lev}(\iota)<\operatorname{rk}(A)$ for all $\iota \in J$.

(c),(d) are trivial, since $\mathcal{I} \neq$ Cut $_{C}$ and $\mathcal{I} \neq \bigvee_{B}^{\iota_{0}}$.

(g) $\mathrm{k}(\mathcal{I})=\mathrm{k}(B) \subseteq \bigcup_{i<m} \mathrm{k}\left(F_{i}\right)=\mathrm{k}(d)$, and since for each RS-sentence $F$ we have $\operatorname{rk}(F)=\omega \cdot \operatorname{lev}(F)+n \in$ $\mathcal{H}^{0}(\mathrm{k}(F))$, we also get $\mathrm{o}(d) \in \mathcal{H}^{0}(\mathrm{k}(d))$.

(f) Note that for every $\mathrm{RS}^{0}$-inference $\mathcal{J}$ we have $\forall i \in|\mathcal{J}|\left(\mathrm{k}\left(\Delta_{i}(\mathcal{J})\right) \subseteq \mathrm{k}(\mathcal{J})\right)$.

Hence by L.1.2 $\mathbf{k}(d[\iota]) \subseteq \mathbf{k}(\operatorname{End}(d[\iota])) \cup \mathbf{k}_{c}(d[\iota])$.

Further $\mathrm{k}(\operatorname{End}(d[\iota])) \stackrel{(\mathrm{b})}{\subseteq} \mathrm{k}(\operatorname{End}(d)) \cup \mathrm{k}\left(B_{\iota}\right) \subseteq \mathrm{k}(\operatorname{End}(d)) \cup \mathrm{k}(\iota)=\mathrm{k}(d) \cup \mathrm{k}(\iota)$.

Therefore it only remains to check that $\mathrm{k}_{c}(d[\iota]) \subseteq \mathcal{H}^{0}(\mathrm{k}(d) \cup \mathrm{k}(\iota))$, i.e., that $\mathrm{k}(\theta) \subseteq \mathcal{H}^{0}(\mathrm{k}(\Pi) \cup \mathrm{k}(\iota))$ for each $\mathrm{Cut}_{\theta}$ or $\bigvee_{A}^{\theta}$ occurring in $d[\iota]$. (Note that $\mathrm{k}(d)=\bigcup_{i<n} \mathrm{k}\left(F_{i}\right)$.)

DEFINITION of $\left(A x_{j}^{*} \Pi\right)[\iota]$ for $\iota \in\left|\operatorname{tp}\left(A x_{j}^{*} \Pi\right)\right|$

To facilitate the verification of (b) in the proof of Theorem 2.1 we will present $\mathrm{RS}^{0}$-derivations in a more traditional manner, namely as proof figures where each node carries a sequence of formulas $A_{1}, \ldots, A_{n}$ and some information indicating the inference by which $A_{1}, \ldots, A_{n}$ has been "derived" from the sequences immediately above $A_{1}, \ldots, A_{n}$.

Example: Let $d=\mathrm{Ax}_{4}^{*}(a \subseteq a)$.

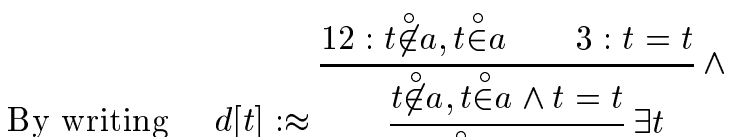

$$
\frac{t \notin i \notin a, t \in a}{t \in a \rightarrow t \in a} \vee^{*}
$$

we actually mean $d[t]:=\bigvee_{t \in a \rightarrow t \in a}^{0} \bigvee_{t \in a \rightarrow t \in a}^{1} \bigvee_{t \in a}^{t} \Lambda_{t \in a \wedge t=t}^{\circ} \mathrm{Ax}_{12}^{*}(t \notin \stackrel{\circ}{\notin} a, t \stackrel{\circ}{\in} a) \mathrm{Ax}_{3}^{*}(t=t)$, if $a \not \equiv \mathrm{L}_{\alpha}$, and $d[t]:=\bigvee_{t \in a}^{t} \mathrm{Ax}_{3}^{*}(t=t)$, if $a \equiv \mathrm{L}_{\alpha}$. Note that $\left(t \stackrel{\circ}{\in} \mathrm{L}_{\alpha} \rightarrow t \in a\right) \equiv t \in a$ and $\left(t \stackrel{\circ}{\in} \mathrm{L}_{\alpha} \wedge t=t\right) \equiv t=t$.

At the same time this representation shows that $\operatorname{End}(d[t]) \subseteq\{t \stackrel{\circ}{\in} a \rightarrow t \in a\}$.

Another example:

The proof figure $\frac{6: \operatorname{tran}\left(\mathrm{L}_{\kappa}\right) \quad 12: \mathrm{L}_{\kappa} \neq a, \mathrm{~L}_{\kappa} \neq a, \neg \operatorname{tran}\left(\mathrm{L}_{\kappa}\right), \operatorname{tran}(a)}{\mathrm{L}_{\kappa} \neq a, \operatorname{tran}(a)} \mathrm{Cut}$

denotes the $\mathrm{RS}^{0}$-derivation $e:=\mathrm{Cut}_{\operatorname{tran}\left(\mathrm{L}_{\kappa}\right)} \mathrm{Ax}_{6}^{*}\left(\operatorname{tran}\left(\mathrm{L}_{\kappa}\right)\right) \mathrm{Ax}_{12}^{*}\left(\mathrm{~L}_{\kappa} \neq a, \mathrm{~L}_{\kappa} \neq a, \neg \operatorname{tran}\left(\mathrm{L}_{\kappa}\right), \operatorname{tran}(a)\right)$ with $\operatorname{End}(e)=\left\{\mathrm{L}_{\kappa} \neq a, \operatorname{tran}(a)\right\}$ and $\mathrm{o}(e)=\omega^{\mathrm{rk}\left(\mathrm{L}_{\kappa} \neq a\right)} \# \omega^{\mathrm{rk}\left(\mathrm{L}_{\kappa} \neq a\right)} \# \omega^{\mathrm{rk}\left(\operatorname{tran}\left(\mathrm{L}_{\kappa}\right)\right)} \# \omega^{\mathrm{rk}(\operatorname{tran}(a))} \# 1$.

Now we start with the definition of $\left(\mathrm{Ax}_{j}^{*} \Pi\right)[\iota]$.

(General) If $d=\mathrm{Ax}_{1}^{*}\left(\forall x \in \mathrm{L}_{\lambda} \forall y \in \mathrm{L}_{\lambda} A(x, y)\right)$ then $d[a]:=\mathrm{Ax}_{1}^{*}\left(\forall y \in \mathrm{L}_{\lambda} A(a, y)\right)$ for $a \in \mathcal{T}_{\lambda}$. 
(Ext1) $d=\operatorname{Ax}_{1}^{*}\left(\forall z \in \mathrm{L}_{\lambda}(a=b \rightarrow(a \in z \rightarrow b \in z))\right)$ with $a, b \in \mathcal{T}_{\lambda}$ :

$d[c]: \approx \frac{12: a \neq b, a \notin c, b \in c}{a=b \rightarrow(a \in c \rightarrow b \in c)} \vee^{*} \quad$ for $c \in \mathcal{T}_{\lambda}$

$(\operatorname{Ext2}) \quad d:=\operatorname{Ax}_{1}^{*}\left(\forall y \in \mathrm{L}_{\lambda}(a=y \rightarrow(\operatorname{Ad}(a) \rightarrow \operatorname{Ad}(y)))\right.$ with $a \in \mathcal{T}_{\lambda}:$

$d[b]: \approx \frac{12: a \neq b, \neg \operatorname{Ad}(a), \operatorname{Ad}(b)}{a=b \rightarrow(\operatorname{Ad}(a) \rightarrow \operatorname{Ad}(b))} \vee^{*} \quad$ for $b \in \mathcal{T}_{\lambda}$

(Pair) $d=\operatorname{Ax}_{1}^{*}\left(\forall y \in \mathrm{L}_{\lambda} \exists z \in \mathrm{L}_{\lambda}(a \in z \wedge y \in z)\right)$ with $a \in \mathcal{T}_{\lambda}$ :

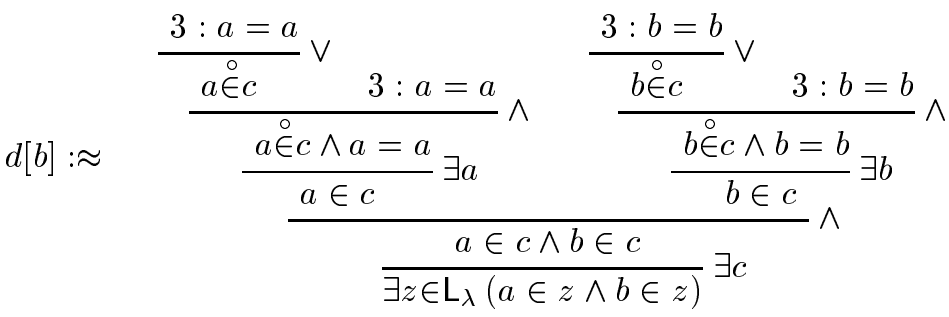

with $c:=\left[x \in \mathrm{L}_{\gamma}: x=a \vee x=b\right], \gamma:=\max \{\operatorname{lev}(a), \operatorname{lev}(b)\}+1=\max (\mathrm{k}(a) \cup \mathrm{k}(b))+1$

(Union) $d=\mathrm{Ax}_{1}^{*}\left(\forall x \in \mathrm{L}_{\lambda} \exists z \in \mathrm{L}_{\lambda} \forall y \in x(y \subseteq z)\right): d[a]: \approx \frac{6: \forall y \in a\left(y \subseteq \mathrm{L}_{\operatorname{lev}(a)}\right)}{\exists z \in \mathrm{L}_{\lambda} \forall y \in a(y \subseteq z)} \exists \mathrm{L}_{\operatorname{lev}(a)}$

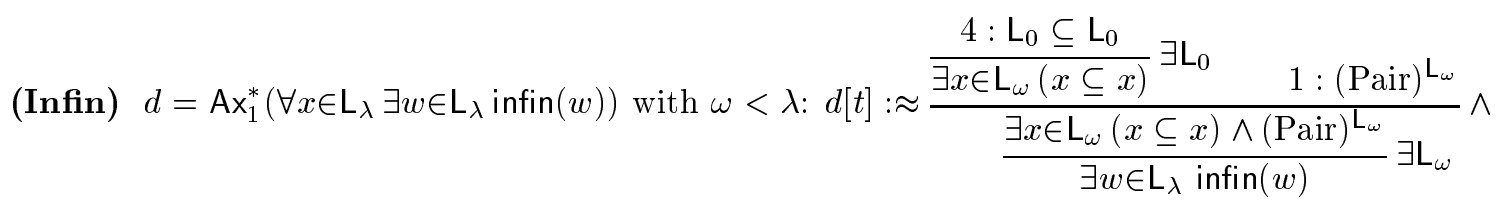

(Found) $d=\mathrm{Ax}_{1}^{*}\left(\forall x_{0} \in \mathrm{L}_{\lambda}\left(G \rightarrow A\left(x_{0}\right)\right)\right)$ with $G \equiv \forall x \in \mathrm{L}_{\lambda}(\forall y \in x A(y) \rightarrow A(x))$ :

$d[a]: \approx \frac{11: \neg G, \forall y \in a A(y) \quad 12: \neg A(a), A(a)}{\frac{\neg G, \forall y \in a A(y) \wedge \neg A(a), A(a)}{\frac{\neg G, A(a)}{G \rightarrow A(a)}} \vee^{*}} \wedge a \quad$ for $a \in \mathcal{T}_{\lambda}$

$\operatorname{rk}(\forall y \in a A(y))<\operatorname{rk}(G)<\operatorname{rk}\left(\forall x_{0} \in \mathrm{L}_{\lambda}\left(G \rightarrow A\left(x_{0}\right)\right)\right)$ and $\operatorname{lev}(a)<\lambda \Rightarrow$

$\mathrm{o}\left(\operatorname{Ax}_{11}^{*}(\neg G, \forall y \in a A(y))\right)=\omega^{\mathrm{rk}(\forall y \in a A(y))} \# \omega \cdot \operatorname{lev}(a)<\omega^{\mathrm{rk}\left(\forall x_{0} \in \mathrm{L}_{\lambda}\left(G \rightarrow A\left(x_{0}\right)\right)\right)} \# \omega \cdot \lambda=\mathrm{o}(d)$

$\left(\Delta_{0}\right.$-Sep $) \quad d=\mathrm{Ax}_{1}^{*}\left(\forall w \in \mathrm{L}_{\lambda} \exists y \in \mathrm{L}_{\lambda}[\forall x \in y(x \in w \wedge F(x)) \wedge \forall x \in w(F(x) \rightarrow x \in y)]\right)$ with $\mathrm{k}(F) \subseteq \lambda$ :

$d[a]: \approx \frac{7: \forall x \in b(x \in a \wedge F(x)) \quad 8: \forall x \in a(F(x) \rightarrow x \in b)}{\frac{\forall x \in b(x \in a \wedge F(x)) \wedge \forall x \in a(F(x) \rightarrow x \in b)}{\exists y \in \mathrm{L}_{\lambda} \forall x \in y(x \in a \wedge F(x)) \wedge \forall x \in a(F(x) \rightarrow x \in y)}} \exists b$

with $b \equiv\left[x \in \mathrm{L}_{\beta}: x \in a \wedge F(x)\right\}, \beta:=\max \{\mathrm{k}(a) \cup \mathrm{k}(F)\}+1$.

Then $\mathrm{k}(b)=\{\beta\} \cup \mathrm{k}(a) \cup \mathrm{k}(F) \subseteq \mathcal{H}^{0}(\mathrm{k}(d) \cup \mathrm{k}(a))$, and $\operatorname{lev}(b)=\beta<\lambda \leq \mathrm{o}(\Pi)$.

(Ad1),(Ad2) $d=\operatorname{Ax}_{1}^{*}\left(\forall x \in \mathrm{L}_{\lambda}[\operatorname{Ad}(x) \rightarrow C(x)]\right): d[a]: \approx \frac{9 \text { or } 10: \neg \operatorname{Ad}(a), C(a), C(a)}{\operatorname{Ad}(a) \rightarrow C(a)} \vee^{*}$ 
$\left(\Delta_{0}\right.$-Col $) \quad d=\mathrm{Ax}_{1}^{*}\left(\forall u \in \mathrm{L}_{\lambda}\left[\forall x \in u \exists y \in \mathrm{L}_{\lambda} A(x, y) \rightarrow \exists w \in \mathrm{L}_{\lambda} \forall x \in u \exists y \in w A(x, y)\right]\right)$ with $\lambda \in \mathrm{R}:$

$d[a]: \approx \frac{12: \neg \forall x \in a \exists y \in \mathrm{L}_{\lambda} A(x, y), \forall x \in a \exists y \in \mathrm{L}_{\lambda} A(x, y)}{\neg \forall x \in a \exists y \in \mathrm{L}_{\lambda} A(x, y), \exists w \in \mathrm{L}_{\lambda} \forall x \in a \exists y \in w A(x, y)} \operatorname{Ref}^{\forall x \in a \exists y \in \mathrm{L}_{\lambda} A(x, y) \rightarrow \exists w \in \mathrm{L}_{\lambda} \forall x \in a \exists y \in w A(x, y)} \mathrm{V}^{*}$

(2) $d=\mathrm{Ax}_{2}^{*}\left(\forall x \in \mathrm{L}_{I} \exists y \in \mathrm{L}_{I}(\operatorname{Ad}(y) \wedge x \in y)\right)$ :

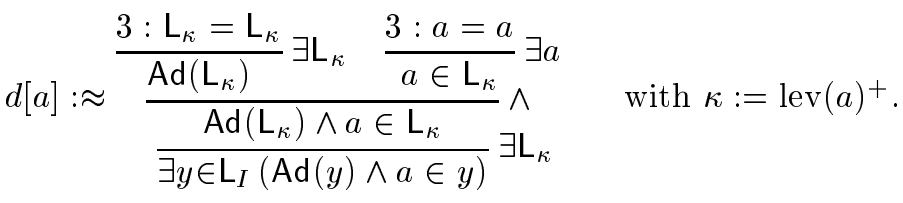

(3) $d=\mathrm{Ax}_{3}^{*}(a=a): d[i]:=\mathrm{Ax}_{4}^{*}(a \subseteq a)$.

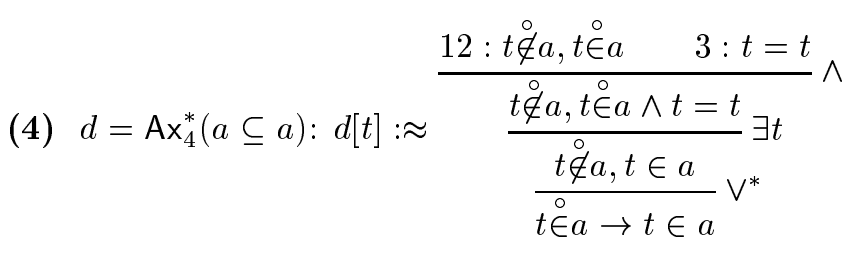

(5) $d=\mathrm{Ax}_{5}^{*}\left(b \subseteq \mathrm{L}_{\alpha}\right)$ with $\operatorname{lev}(b) \leq \alpha: d[t]: \approx \frac{\frac{3: t=t}{t \in \mathrm{L}_{\alpha}} \exists t}{t \in \mathrm{o}^{\circ} b t \in \mathrm{L}_{\alpha}} \vee$

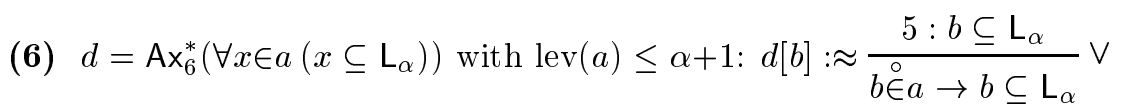

(7) $d=\mathrm{Ax}_{7}^{*}(\forall x \in b(x \in a \wedge F(x)))$ with $b \equiv\left[x \in \mathrm{L}_{\beta}: x \in a \wedge F(x)\right]$ :

$d[t]: \approx \frac{12: \neg(t \in a \wedge F(t)), t \in a \wedge F(t)}{t \stackrel{\circ}{\in} b \rightarrow t \in a \wedge F(t)} \vee^{*}$

(8) $d=\mathrm{Ax}_{8}^{*}(\forall x \in a(F(x) \rightarrow x \in b))$ with $b \equiv\left[x \in \mathrm{L}_{\beta}: x \in a \wedge F(x)\right]$ :

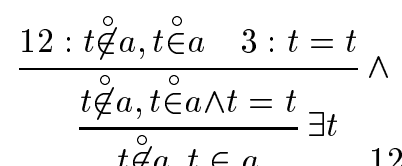

$$
\begin{aligned}
& \frac{t \dot{\notin} a, t \in a}{12: \neg F(t), F(t)} \wedge \\
& \frac{t \stackrel{\circ}{\dot{\notin} a, \neg F(t), t \in a \wedge F(t)} \wedge}{3: t=t} \wedge
\end{aligned}
$$

(9) $d=\mathrm{Ax}_{9}^{*}(\neg \operatorname{Ad}(a), \operatorname{tran}(a), \operatorname{tran}(a))$ :

$d\left[\mathrm{~L}_{\kappa}\right]: \approx \frac{6: \operatorname{tran}\left(\mathrm{L}_{\kappa}\right) \quad 12: \mathrm{L}_{\kappa} \neq a, \mathrm{~L}_{\kappa} \neq a, \neg \operatorname{tran}\left(\mathrm{L}_{\kappa}\right), \operatorname{tran}(a)}{\mathrm{L}_{\kappa} \neq a, \operatorname{tran}(a)} \mathrm{Cut} \quad$ for $\kappa \leq \operatorname{lev}(a)$

$\operatorname{rk}\left(\mathrm{L}_{\kappa} \neq a\right)<\operatorname{rk}(\neg \operatorname{Ad}(a)) \& \operatorname{rk}\left(\operatorname{tran}\left(\mathrm{L}_{\kappa}\right)\right) \leq \operatorname{rk}(\operatorname{tran}(a)) \Rightarrow$

$\mathrm{o}\left(\mathrm{L}_{\kappa} \neq a, \mathrm{~L}_{\kappa} \neq a, \neg \operatorname{tran}\left(\mathrm{L}_{\kappa}\right), \operatorname{tran}(a)\right)<\mathrm{o}(\neg \mathrm{Ad}(a), \operatorname{tran}(a), \operatorname{tran}(a))$ 
(10) $d=\operatorname{Ax}_{10}^{*}\left(\neg \operatorname{Ad}(a), \psi^{a}, \psi^{a}\right): d\left[\mathrm{~L}_{\kappa}\right]: \approx \frac{1: \psi^{\kappa} \quad 12: \mathrm{L}_{\kappa} \neq a, \ldots, \mathrm{L}_{\kappa} \neq a, \neg \psi^{\kappa}, \psi^{a}}{\mathrm{~L}_{\kappa} \neq a, \psi^{a}} \mathrm{Cut}$ or $d\left[\mathrm{~L}_{\kappa}\right]: \approx \frac{\frac{4: \mathrm{L}_{0} \subseteq \mathrm{L}_{0}}{\exists x \in \mathrm{L}_{\omega}(x \subseteq x)} \exists \mathrm{L}_{0} \quad 1:(\text { Pair })^{\mathrm{L}_{\omega}}}{\frac{\exists x \in \mathrm{L}_{\omega}(x \subseteq x) \wedge(\text { Pair })^{\mathrm{L}_{\omega}}}{\frac{\psi^{\kappa}}{\mathrm{L}_{\omega}}} \wedge \quad 12: \mathrm{L}_{\kappa} \neq a, \neg \psi^{\kappa}, \psi^{a}}$ Cut

if $\psi$ is the axiom (Infin), i.e., $\psi \equiv \exists w\left(\exists x \in w(x \subseteq x) \wedge{\left.\text { (Pair })^{w}\right)}^{w}\right.$

(11) $d=\operatorname{Ax}_{11}^{*}(\neg G, \forall x \in a A(x))$ with $G: \equiv \forall x \in \mathrm{L}_{\alpha}(\forall y \in x A(y) \rightarrow A(x))$ and $\operatorname{lev}(a) \leq \alpha$ :

$d[t]: \approx \quad \frac{11: \neg G, \forall y \in t A(y) \quad 12: \neg A(t), A(t)}{\frac{\neg G, \forall y \in t A(y) \wedge \neg A(t), A(t)}{\neg \frac{\neg G, A(t)}{\neg G, t \stackrel{\circ}{\in} a \rightarrow A(t)}} \vee t} \wedge \quad$ for $t \in \mathcal{T}_{a}$

$\operatorname{rk}(\forall y \in t A(y)) \leq \operatorname{rk}(\forall x \in a A(x)) \& \operatorname{lev}(t)<\operatorname{lev}(a) \Rightarrow$

$\mathrm{o}\left(\operatorname{Ax}_{11}^{*}(\neg G, \forall y \in t A(y))\right)=\omega^{\mathrm{rk}(\forall y \in t A(y))} \# \omega \cdot \operatorname{lev}(t)<\omega^{\mathrm{rk}(\forall x \in a A(x))} \# \omega \cdot \operatorname{lev}(a)=\mathrm{o}(d)$ and

$\mathrm{o}\left(\mathrm{Ax}_{12}^{*}(\neg A(t), A(t))\right)=\omega^{\mathrm{rk}(A(t))} \cdot 2<\mathrm{o}(d)$

(12) $d=\operatorname{Ax}_{12}^{*}\left(\left[s_{1} \neq t_{1}\right], \ldots,\left[s_{n} \neq t_{n}\right], \neg A(\vec{s}), A(\vec{t})\right)$ :

We distinguish cases according to the shape of $A$. It suffices to consider the three cases $A \equiv x_{1} \in x_{2}$, $A \equiv \operatorname{Ad}\left(x_{1}\right), A \equiv \exists y \in x_{2} B\left(x_{1}, y\right)$ with $\left[s_{i} \neq t_{i}\right] \equiv s_{i} \neq t_{i}$. The remaining cases are treated similarly.

$d=\operatorname{Ax}_{12}^{*}\left(s_{1} \neq t_{1}, s_{2} \neq t_{2}, s_{1} \notin s_{2}, t_{1} \in t_{2}\right)$ :

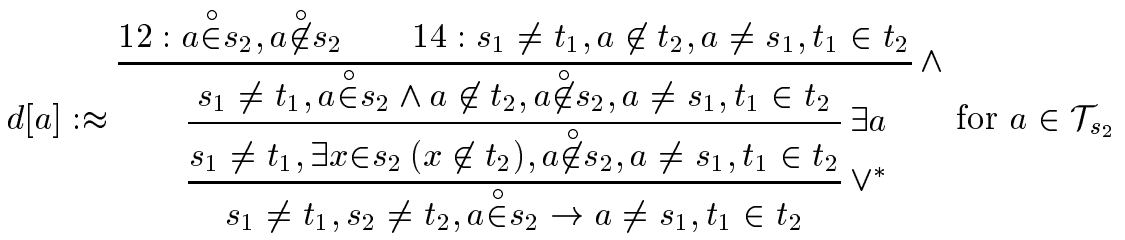

$d=\operatorname{Ax}_{12}^{*}(a \neq b, \neg \operatorname{Ad}(a), \operatorname{Ad}(b))$ :

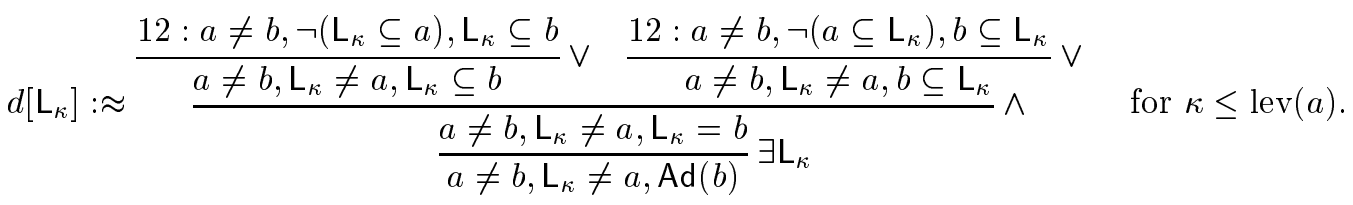

Let $c \in\{a, b\}$ such that $\operatorname{rk}(c)=\max \{\operatorname{rk}(a), \operatorname{rk}(b)\}$.

$\operatorname{rk}\left(a \subseteq \mathrm{L}_{\kappa}\right)<\operatorname{rk}(\operatorname{Ad}(a)) \leq \operatorname{rk}(\operatorname{Ad}(c)) \& \operatorname{rk}\left(b \subseteq \mathrm{L}_{\kappa}\right) \stackrel{(*)}{<} \operatorname{rk}(\operatorname{Ad}(c)) \Rightarrow$

$\omega^{\mathrm{rk}\left(a \subseteq \mathrm{L}_{\kappa}\right)} \# \omega^{\mathrm{rk}\left(b \subseteq \mathrm{L}_{\kappa}\right)}<\omega^{\mathrm{rk}(\operatorname{Ad}(c))}<\omega^{\mathrm{rk}(\operatorname{Ad}(a))} \# \omega^{\mathrm{rk}(\operatorname{Ad}(b))}$

$(*)$ :

1. $\operatorname{lev}(a) \leq \operatorname{lev}(b) \Rightarrow \operatorname{rk}\left(b \subseteq \mathrm{L}_{\kappa}\right)<\operatorname{rk}(\operatorname{Ad}(b)) \leq \operatorname{rk}(\operatorname{Ad}(c))$

2. $\operatorname{lev}(b)<\operatorname{lev}(a) \Rightarrow \operatorname{rk}(b)<\omega \cdot(\operatorname{lev}(b)+1) \leq \omega \cdot \operatorname{lev}(a) \leq \operatorname{rk}(a) \Rightarrow \operatorname{rk}\left(b \subseteq \mathrm{L}_{\kappa}\right) \leq \operatorname{rk}\left(a \subseteq \mathrm{L}_{\kappa}\right)<\operatorname{rk}(\operatorname{Ad}(a))$ 
$d=\mathrm{Ax}_{12}^{*}\left(s_{1} \neq t_{1}, s_{2} \neq t_{2}, \neg A\left(s_{1}, s_{2}\right), A\left(t_{1}, t_{2}\right)\right)$ with $A\left(x_{1}, x_{2}\right) \equiv \exists y \in x_{2} B\left(x_{1}, y\right)$ :

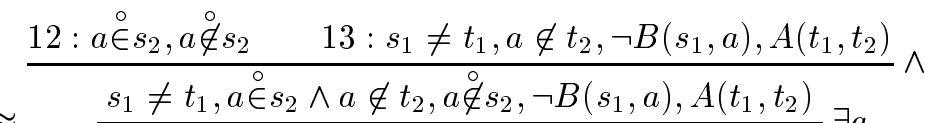

$$
\begin{aligned}
& d[a]: \approx \quad \frac{s_{1} \neq t_{1}, a \in s_{2} \wedge a \notin t_{2}, a \notin s_{2}, \neg B\left(s_{1}, a\right), A\left(t_{1}, t_{2}\right)}{s_{1} \neq t_{1}, a c s_{2}\left(x \notin t_{2}\right), a \notin s_{2}, \neg B\left(s_{1}, a\right), A\left(t_{1}, t_{2}\right)} \exists a \\
& \frac{s_{1} \neq t_{1}, \exists x \in s_{2}\left(x \notin t_{2}\right), a \stackrel{\circ}{\notin} s_{2}, \neg B\left(s_{1}, a\right), A\left(t_{1}, t_{2}\right)}{s_{1} \neq t_{1}, s_{2} \neq t_{2}, a \stackrel{\circ}{\epsilon} s_{2} \rightarrow \neg B\left(s_{1}, a\right), A\left(t_{1}, t_{2}\right)} \vee^{*}
\end{aligned}
$$

(13) $d=\mathrm{Ax}_{13}^{*}\left(s_{1} \neq t_{1}, a \notin t_{2}, \neg B\left(s_{1}, a\right), A\left(t_{1}, t_{2}\right)\right)$ with $A\left(x_{1}, x_{2}\right) \equiv \exists y \in x_{2} B\left(x_{1}, y\right)$ :

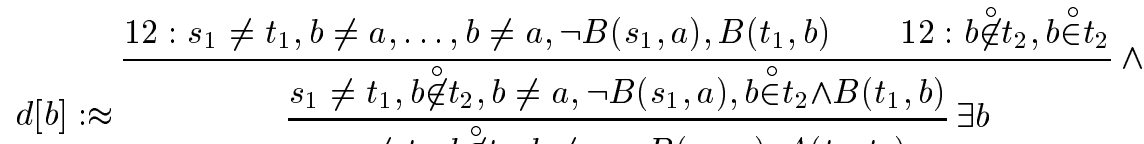

$$
\begin{aligned}
& \frac{s_{1} \neq t_{1}, b \stackrel{\circ}{\notin} t_{2}, b \neq a, \neg B\left(s_{1}, a\right), A\left(t_{1}, t_{2}\right)}{s_{1} \neq t_{1}, b \stackrel{\circ}{\oplus} t_{2} \rightarrow b \neq a, \neg B\left(s_{1}, a\right), A\left(t_{1}, t_{2}\right)} \vee^{*}
\end{aligned}
$$

(14) $d=\mathrm{Ax}_{14}^{*}\left(s_{1} \neq t_{1}, a \notin t_{2}, a \neq s_{1}, t_{1} \in t_{2}\right)$ :

$$
\begin{aligned}
& \frac{12: s_{1} \neq t_{1}, b \neq a, \neg\left(a \subseteq s_{1}\right), b \subseteq t_{1}}{\underline{s_{1} \neq t_{1}, b \neq a, a \neq s_{1}, b \subseteq t_{1}} \vee \quad \frac{12: s_{1} \neq t_{1}, b \neq a, \neg\left(s_{1} \subseteq a\right), t_{1} \subseteq b}{s_{1} \neq t_{1}, b \neq a, a \neq s_{1}, t_{1} \subseteq b} \wedge} \vee \\
& d[b]: \approx
\end{aligned}
$$

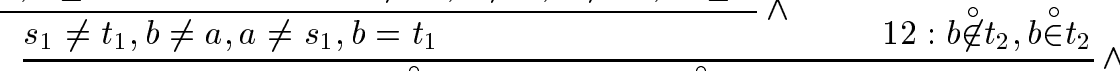

$$
\begin{aligned}
& \underline{s_{1} \neq t_{1}, b \notin t_{2}, b \neq a, a \neq s_{1}, b \stackrel{\circ}{\epsilon} t_{2} \wedge b=t_{1}} \exists b \\
& \frac{s_{1} \neq t_{1}, b \stackrel{\circ}{\notin} t_{2}, b \neq a, a \neq s_{1}, t_{1} \in t_{2}}{s_{1} \neq t_{1}, b \stackrel{\circ}{\in} t_{2} \rightarrow b \neq a, a \neq s_{1}, t_{1} \in t_{2}} \vee^{*}
\end{aligned}
$$

(15) $d=\mathrm{Ax}_{15}^{*}\left(\forall x \in \mathrm{L}_{\omega} \exists u \in \mathrm{L}_{\omega}(\exists y \in u(x \in y) \wedge \mathcal{A}(u))\right)$ :

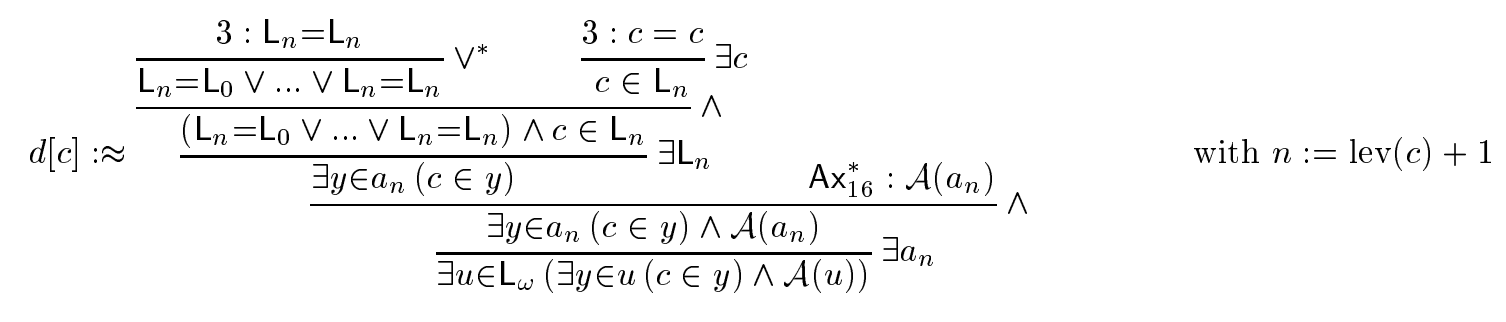

(16) $d=\operatorname{Ax}_{16}^{*}\left(\mathcal{A}\left(a_{n}\right)\right)$ :

Remember that $a_{n} \equiv\left[x \in \mathrm{L}_{n+1}: x=\mathrm{L}_{0} \vee \ldots \vee x=\mathrm{L}_{n}\right]$ and $\mathcal{A}\left(a_{n}\right) \equiv \forall x \in a_{n}\left(\mathcal{E}(x) \vee \mathcal{B}\left(a_{n}, x\right)\right)$ with $\mathcal{E}(x) \equiv \forall y \in x(y \neq y), \mathcal{B}\left(a_{n}, x\right) \equiv \exists x_{0} \in a_{n}\left(x_{0} \in x \wedge \forall y \in x\left(y \subseteq x_{0}\right)\right)$.

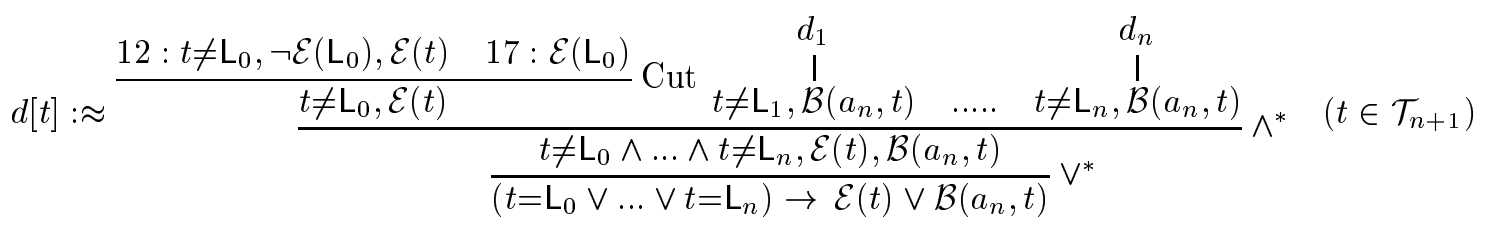

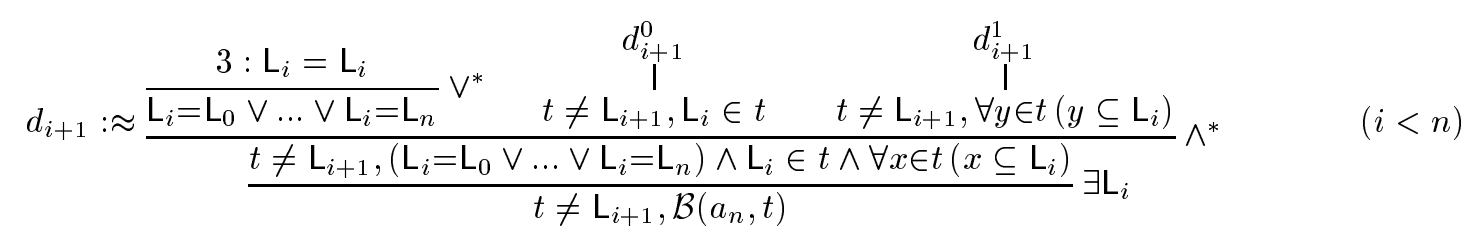


$d_{i+1}^{0}: \approx \frac{12: t \neq \mathrm{L}_{i+1}, \mathrm{~L}_{i} \notin \mathrm{L}_{i+1}, \mathrm{~L}_{i} \in t \quad \frac{3: \mathrm{L}_{i}=\mathrm{L}_{i}}{\mathrm{~L}_{i} \in \mathrm{L}_{i+1}}}{t \neq \mathrm{L}_{i+1}, \mathrm{~L}_{i} \in t} \mathrm{Cut}$

$d_{i+1}^{1}: \approx \frac{12: t \neq \mathrm{L}_{i+1}, \neg \forall y \in \mathrm{L}_{i+1}\left(y \subseteq \mathrm{L}_{i}\right), \forall y \in t\left(y \subseteq \mathrm{L}_{i}\right) \quad 6: \forall y \in \mathrm{L}_{i+1}\left(y \subseteq \mathrm{L}_{i}\right)}{t \neq \mathrm{L}_{i+1}, \forall y \in t\left(y \subseteq \mathrm{L}_{i}\right)}$ Cut

(17) $d=\operatorname{Ax}_{17}^{*}\left(\forall y \in \mathrm{L}_{0}(y \neq y)\right):|\operatorname{tp}(d)|=\mathcal{T}_{0}=\emptyset$.

\section{$\S 3$ The notation system $\mathbf{R S}^{+}$}

Now we extend $\mathbf{R S}^{0}$ to a notation system $\mathbf{R S}^{+}$which also contains notations for $\mathrm{RS}^{\infty}$-derivations of all sequents $\phi_{1}^{\mathrm{L}_{I}}, \ldots, \phi_{n}^{\mathrm{L}_{I}}$ where $\phi_{1} \vee \ldots \vee \phi_{n}$ is a logically valid $\mathcal{L}_{\mathrm{Ad}^{-}}$-formula.

\section{Inductive definition of $\mathrm{RS}_{\lambda}$-formulas}

1. If $u, v \in \mathcal{T}_{\lambda} \cup \operatorname{Var}$ then $u \in v, u \notin v, \operatorname{Ad}(u), \neg \operatorname{Ad}(u)$ are $\operatorname{RS}_{\lambda}$-formulas.

2. If $A, B$ are $\mathrm{RS}_{\lambda}$-formulas then so are $A \wedge B, A \vee B, \forall x \in \mathrm{L}_{\lambda} A, \exists x \in \mathrm{L}_{\lambda} A$.

3. If $A$ is an $\mathrm{RS}_{\lambda}$-formula and $x \neq u \in \mathcal{T}_{\lambda} \cup \operatorname{Var}$ then $\forall x \in u A$ and $\exists x \in u A$ are $\mathrm{RS}_{\lambda}$-formulas.

In the following $A, B, C, D$ always denote $\mathrm{RS}_{I}$-formulas!

\section{Definition of $\operatorname{rk}_{0}(A)$}

1. $\operatorname{rk}_{0}(A):=\operatorname{rk}_{0}(\neg A):=0$ if $A$ atomic,

2. $\operatorname{rk}_{0}\left(A_{\wedge}^{\vee} B\right):=\max \left\{\operatorname{rk}_{0}(A), \operatorname{rk}_{0}(B)\right\}+1$,

3. $\operatorname{rk}_{0}\left({ }_{\forall}^{\exists} x \in a A\right):=\operatorname{rk}_{0}(A)+2$.

Lemma 3.1 $\operatorname{rk}(A)<I+\operatorname{rk}_{0}(A)$ for each $\mathrm{RS}_{I}$-sentence $A$.

Proof:

1. $A$ atomic: Then $\operatorname{lev}(A)<I$ and thus $\operatorname{rk}(A)=\omega \cdot \operatorname{lev}(A)+n<I$.

2. $\operatorname{rk}(A \wedge B) \stackrel{\mathrm{IH}}{<} \max \left\{I+\mathrm{rk}_{0}(A), I+\mathrm{rk}_{0}(B)\right\}+1=I+\max \left\{\mathrm{rk}_{0}(A), \mathrm{rk}_{0}(B)\right\}+1=I+\mathrm{rk}_{0}(A \wedge B)$.

3. $\operatorname{rk}(\forall x \in a A(x))=\max \left\{\operatorname{rk}(a), \operatorname{rk}\left(A\left(\mathrm{~L}_{0}\right)\right)+2\right\} \stackrel{\mathrm{IH}}{<} I+\operatorname{rk}_{0}\left(A\left(\mathrm{~L}_{0}\right)\right)+2=I+\mathrm{rk}_{0}(\forall x \in a A(x))$.

(Note that $a \in \mathcal{T}_{I} \cup\left\{\mathrm{L}_{I}\right\}$ and therefore $\operatorname{rk}(a) \leq I$.)

\section{Definition of the finitary proof system $\mathrm{RS}^{1}$}

The proof system $\mathrm{RS}^{1}$ consists of the following inferences:

$\left(\mathrm{Ax}_{\neg A, A}^{I}\right) \neg A, A$

$\left(\mathrm{Ax}_{\forall x \in u A}^{I}\right) \quad \neg \forall x \in \mathrm{L}_{I}(x \in u \rightarrow A), \forall x \in u A \quad$ if $u \in \mathcal{T}_{I} \cup \operatorname{Var}, u \neq x$

$\left(\mathrm{Ax}_{\exists x \in u A}^{I}\right) \quad \neg \exists x \in \mathrm{L}_{I}(x \in u \wedge A), \exists x \in u A \quad$ if $u \in \mathcal{T}_{I} \cup \operatorname{Var}, u \neq x$

$\left(\bigwedge_{A_{0} \wedge A_{1}}\right) \frac{A_{0} A_{1}}{A_{0} \wedge A_{1}} \quad\left(\bigvee_{A_{0} \vee A_{1}}^{k}\right) \frac{A_{k}}{A_{0} \vee A_{1}}$

$\left(\forall_{\forall x \in \mathrm{L}_{I} A}^{y}\right) \frac{A_{x}(y)}{\forall x \in \mathrm{L}_{I} A} ! y ! \quad\left(\exists_{\exists x \in \mathrm{L}_{I} A}^{v}\right) \frac{A_{x}(v)}{\exists x \in \mathrm{L}_{I} A} \quad$ if $v \in \mathcal{T}_{I} \cup$ Var

$\left(\mathrm{Cut}_{C}\right) \frac{C \quad \neg C}{\emptyset}$ 
$\mathrm{k}(\mathcal{I}):= \begin{cases}\mathrm{k}(C) & \text { if } \mathcal{I}=\text { Cut }_{C} \\ \mathrm{k}(A) \cup \mathrm{k}\left(\iota_{0}\right) & \text { if } \mathcal{I}=\exists_{A}^{\iota_{0}} \text { with } \iota_{0} \in \mathcal{T}_{I} \\ \mathrm{k}(\Delta(\mathcal{I})) & \text { otherwise }\end{cases}$

Notation: For the rest of this section $d, d_{i}$ denote $\mathrm{RS}^{1}$-derivations.

Definition of $\mathrm{o}(d)$ and $\operatorname{deg}(d)$

$\mathrm{o}\left(\mathrm{Ax}_{\neg A, A}^{I}\right):=\mathrm{o}\left(\mathrm{Ax}_{Q x \in u A}^{I}\right):=\omega^{I+\mathrm{rk}_{0}(A)+2}, \quad \mathrm{o}\left(\mathcal{I} d_{0} \ldots d_{n}\right):=\max \left\{\mathrm{o}\left(d_{0}\right), \ldots, \mathrm{o}\left(d_{n}\right)\right\}+1$
$\operatorname{deg}\left(\mathcal{I} d_{0} \ldots d_{n-1}\right):= \begin{cases}\max \left\{I+\mathrm{rk}_{0}(C), \operatorname{deg}\left(d_{0}\right), \operatorname{deg}\left(d_{1}\right)\right\} & \text { if } \mathcal{I}=\mathrm{Cut}_{C} \\ \sup _{i<n} \operatorname{deg}\left(d_{i}\right) & \text { otherwise }\end{cases}$

Definition of $\mathrm{FV}(d)$

$\mathrm{FV}\left(\mathcal{I} d_{0} \ldots d_{n-1}\right):=\mathrm{FV}(\mathcal{I}) \cup \bigcup_{i<n}\left(\mathrm{FV}\left(d_{i}\right) \backslash \operatorname{Eig}(\mathcal{I})\right)$ with

$\operatorname{FV}(\mathcal{I}):=\left\{\begin{array}{ll}\operatorname{FV}(\Delta(\mathcal{I})) \cup \mathrm{FV}(v) & \text { if } \mathcal{I}=\exists_{A}^{v} \\ \operatorname{FV}(\Delta(\mathcal{I})) & \text { otherwise }\end{array} \quad\right.$ and $\quad \operatorname{Eig}(\mathcal{I}):= \begin{cases}\{y\} & \text { if } \mathcal{I}=\bigwedge_{A}^{y} \\ \emptyset & \text { otherwise }\end{cases}$

Remark: $\operatorname{Eig}(\mathcal{I}) \cap \operatorname{FV}(\mathcal{I})=\emptyset$.

Definition: A derivation $d$ is called closed if $\operatorname{FV}(d)=\emptyset$.

\section{Substitution}

\section{Definition of $d(z / t)$}

$\left(\mathcal{I} d_{0} \ldots d_{n-1}\right)(z / t)= \begin{cases}\mathcal{I} d_{0} \ldots d_{n-1} & \text { if } \operatorname{Eig}(\mathcal{I})=\{z\} \\ \mathcal{I}(z / t) d_{0}(z / t) \ldots d_{n-1}(z / t) & \text { otherwise }\end{cases}$

where

$\mathrm{Ax}_{\neg A, A}^{I}(z / t):=\mathrm{Ax}_{\neg A_{z}(t), A_{z}(t)}^{I}, \mathrm{Ax}_{Q x \in u A}^{I}(z / t):=\operatorname{Ax}_{(Q x \in u A)_{z}(t)}^{I}$,

$\forall_{C}^{y}(z / t):=\forall_{C_{z}(t)}^{y}, \exists_{C}^{v}(z / t):=\exists_{C_{z}(t)}^{v_{z}(t)}$,

$\bigwedge_{A}(z / t):=\bigwedge_{A_{z}(t)}, \bigvee_{A}^{k}(z / t):=\bigvee_{A_{z}(t)}^{k}, \operatorname{Cut}_{C}(z / t):=\operatorname{Cut}_{C_{z}(t)}$.

\section{Lemma 3.2}

If $d$ is an $\mathrm{RS}^{1}$-derivation and $t \in \mathcal{T}_{I}$ then $d(z / t)$ is an $\mathrm{RS}^{1}$-derivation with

$\operatorname{End}(d(z / t)) \subseteq \operatorname{End}(d)_{z}(t), \operatorname{deg}(d(z / t))=\operatorname{deg}(d), o(d(z / t))=o(d), \mathrm{k}(d(z / t)) \subseteq \mathrm{k}(d) \cup \mathrm{k}(t)$.

Proof: straightforward.

\section{Lemma 3.3}

a) $\mathrm{FV}(\operatorname{End}(d)) \subseteq \operatorname{FV}(d)$,

b) $\operatorname{FV}(d(z / t))=\mathrm{FV}(d) \backslash\{z\}$, if $t \in \mathcal{T}_{I}$.

Proof: Let $d=\mathcal{I} d_{0} \ldots d_{n-1}$.

a) $\operatorname{End}(d)=\Delta(\mathcal{I}) \cup \bigcup_{i<n}\left(\operatorname{End}\left(d_{i}\right) \backslash \Delta_{i}(\mathcal{I})\right)$ and $\operatorname{FV}(\operatorname{End}(d)) \cap \operatorname{Eig}(\mathcal{I})=\emptyset(*)$.

$\mathrm{FV}(\Delta(\mathcal{I})) \subseteq \mathrm{FV}(\mathcal{I}) \subseteq \mathrm{FV}(d)$.

$\mathrm{FV}\left(\operatorname{End}\left(d_{i}\right) \backslash \Delta_{i}(\mathcal{I})\right) \stackrel{(*)}{\subseteq} \mathrm{FV}\left(\operatorname{End}\left(d_{i}\right)\right) \backslash \operatorname{Eig}(\mathcal{I}) \stackrel{\mathrm{IH}}{\subseteq} \mathrm{FV}\left(d_{i}\right) \backslash \operatorname{Eig}(\mathcal{I}) \subseteq \mathrm{FV}(d)$.

b) Abb.: $\mathcal{I}^{\prime}:=\mathcal{I}(z / t), d^{\prime}:=d(z / t)$.

1. $\operatorname{Eig}(\mathcal{I})=\{z\}$ : Then $d^{\prime}=d$ and $z \notin \mathrm{FV}(d)$. Hence $\mathrm{FV}\left(d^{\prime}\right)=\mathrm{FV}(d)=\mathrm{FV}(d) \backslash\{z\}$. 
2. Otherwise: Then $d^{\prime}=\mathcal{I}^{\prime} d_{0}^{\prime} \ldots d_{n-1}^{\prime}$, and by $\operatorname{IH~FV}\left(d_{i}^{\prime}\right)=\mathrm{FV}\left(d_{i}\right) \backslash\{z\}$.

Moreover one easily verifies that $\mathrm{FV}\left(\mathcal{I}^{\prime}\right)=\mathrm{FV}(\mathcal{I}) \backslash\{z\}$.

Hence $\mathrm{FV}\left(d^{\prime}\right)=\mathrm{FV}\left(\mathcal{I}^{\prime}\right) \cup \bigcup_{i}\left(\mathrm{FV}\left(d_{i}^{\prime}\right) \backslash \operatorname{Eig}\left(\mathcal{I}^{\prime}\right)\right) \stackrel{\mathrm{IH}}{=}$

$(\mathrm{FV}(\mathcal{I}) \backslash\{z\}) \cup \bigcup_{i}\left(\left(\mathrm{FV}\left(d_{i}\right) \backslash\{z\}\right) \backslash \operatorname{Eig}(\mathcal{I})\right)=$

$\left(\left(\mathrm{FV}(\mathcal{I}) \cup \bigcup_{i}\left(\mathrm{FV}\left(d_{i}\right) \backslash \operatorname{Eig}(\mathcal{I})\right)\right) \backslash\{z\}=\mathrm{FV}(d) \backslash\{z\}\right.$.

\section{Lemma 3.4}

a) Every $\mathrm{RS}^{1}$-derivation $d$ can be transformed into an $\mathrm{RS}^{1}$-derivation $d^{\prime}$ with

$\operatorname{End}\left(d^{\prime}\right) \subseteq \operatorname{End}(d), \operatorname{deg}\left(d^{\prime}\right)=\operatorname{deg}(d), o\left(d^{\prime}\right)=\mathrm{o}(d)$, and $\mathrm{FV}(d)=\mathrm{FV}(\operatorname{End}(d))$

b) If $d=\mathcal{I} d_{0} \ldots d_{n-1}$ is closed and $\operatorname{Eig}(\mathcal{I})=\emptyset$ then $d_{0}, \ldots, d_{n-1}$ are closed.

c) If $d=\mathcal{I} d_{0}$ is closed and $\operatorname{Eig}(\mathcal{I})=\{x\}$ then $d_{0}(x / t)$ is closed for each $t \in \mathcal{T}_{I}$.

Proof:

a) Definition of $d^{\prime}$ by recursion on the cardinality of $\mathrm{FV}(d)$ :

If $\mathrm{FV}(d) \backslash \mathrm{FV}(\operatorname{End}(d))=\emptyset$ then $d^{\prime}:=d$, and the claim follows by L.3.3a.

Now assume that $x \in \mathrm{FV}(d) \backslash \mathrm{FV}(\operatorname{End}(d))$. Then for $d_{1}:=d\left(x / \mathrm{L}_{0}\right)$ we have $\operatorname{End}\left(d_{1}\right) \subseteq \operatorname{End}(d)_{x}\left(\mathrm{~L}_{0}\right)=\operatorname{End}(d)$, $\operatorname{deg}\left(d_{1}\right)=\operatorname{deg}(d), \mathrm{o}\left(d_{1}\right)=\mathrm{o}(d)$, and (by L.3.3b) $\mathrm{FV}\left(d_{1}\right)=\mathrm{FV}(d) \backslash\{x\}$. Hence the claim follows by IH.

b) $\mathrm{FV}\left(d_{i}\right) \subseteq \mathrm{FV}(d) \cup \operatorname{Eig}(\mathcal{I})$.

c) $\mathrm{FV}\left(d_{0}\right) \subseteq \mathrm{FV}(d) \cup\{x\}=\{x\} \stackrel{3.3 \mathrm{~b}}{\Rightarrow} \mathrm{FV}\left(d_{0}(x / t)\right)=\mathrm{FV}\left(d_{0}\right) \backslash\{x\}=\emptyset$.

\section{Definition of the notation system RS $^{+}$}

$\mathbf{R S}^{+}:=\left(\mathcal{D}^{+}, \mathrm{o}, \mathrm{deg}, \mathrm{tp},[]\right)$ extends the system $\mathbf{R S}^{0}$ :

$\mathcal{D}^{+}:=\mathcal{D}_{0} \cup \mathcal{D}_{1}$, where $\mathcal{D}_{0}:=$ set of all $\mathrm{RS}^{0}$-derivations, $\mathcal{D}_{1}:=$ set of all closed $\mathrm{RS}^{1}$-derivations.

Note that $\mathcal{D}_{0} \cap \mathcal{D}_{1}:=\emptyset$, and that every $\mathrm{RS}^{0}$-derivation is closed!

For $d \in \mathcal{D}_{0}$, the entities $\mathrm{o}(d), \operatorname{deg}(d), \operatorname{tp}(d), d[\iota]$ have been defined in $\S 2$ (Definition of $\mathbf{R S}^{0}$ );

for $d \in \mathcal{D}_{1}, o(d)$ and $\operatorname{deg}(d)$ are defined above.

So it remains to define $\operatorname{tp}(d)$ and $d[\iota]$ for $d \in \mathcal{D}_{1}$ :

If $A \simeq \bigwedge\left(A_{\iota}\right)_{\iota \in J}$ then

$\operatorname{tp}\left(\mathrm{Ax}_{\neg A, A}^{I}\right):=\operatorname{tp}\left(\mathrm{Ax}_{A, \neg A}^{I}\right):=\bigwedge_{A}$ and $\left(\mathrm{Ax}_{\neg A, A}^{I}\right)[\iota]:=\left(\mathrm{Ax}_{A, \neg A}^{I}\right)[\iota]:=\bigvee_{\neg A}^{\iota} \mathrm{Ax}_{13}^{*}\left(\neg A_{\iota}, A_{\iota}\right)$

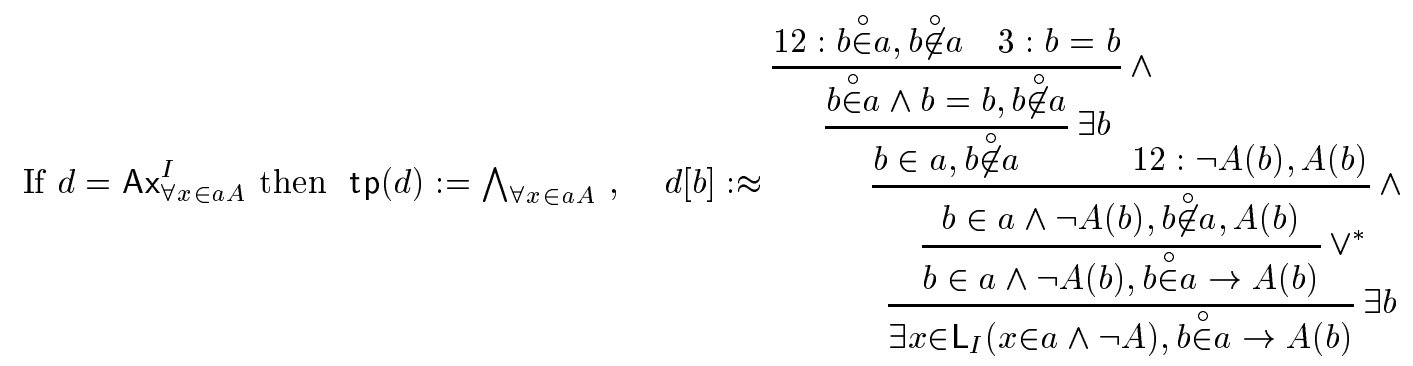

If $d=A x_{\exists x \in a A}^{I}$ then $\operatorname{tp}(d):=\bigwedge_{\forall x \in \mathrm{L}_{I}(x \in a \rightarrow \neg A)}, \quad d[b]: \approx \frac{13: b \notin a, \neg A(b), \exists x \in a A}{b \in a \rightarrow \neg A(b), \exists x \in a A} \vee^{*}$

If $d=\forall_{\forall x \in \mathrm{L}_{I} A}^{y} d_{0}$ then $\operatorname{tp}(d):=\bigwedge_{\forall x \in \mathrm{L}_{I} A}, d[t]:=d_{0}(y / t)$. 
If $d=\exists_{\exists x \in \mathrm{L}_{I} A}^{t} d_{0}$ then $\operatorname{tp}(d):=\bigvee_{\exists x \in \mathrm{L}_{I} A}^{t}, d[0]:=d_{0}$.

If $d=\mathcal{I} d_{0} \ldots d_{n}$ with $\mathcal{I}=\bigwedge_{A_{0} \wedge A_{1}}$ or $\mathcal{I}=\bigvee_{A_{0} \vee A_{1}}^{k}$ or $\mathcal{I}=\mathrm{Cut}_{C}$ then $\operatorname{tp}(d):=\mathcal{I}$ and $d[i]:=d_{i}$.

\section{Theorem 3.5}

$\mathbf{R S}^{+}$is a notation system for $\mathrm{RS}^{\infty}$-derivations, and is controlled by $\mathcal{H}^{0}$.

Proof:

Since in $\S 2$ we have already proved that $\mathbf{R S}^{0}$ is a notation system controlled by $\mathcal{H}^{0}$, it remains to verify conditions (a),..,(f) for $d \in \mathcal{D}_{1}$. Most of that is easy or trivial.

Some hints:

Assume $d=\mathcal{J} d_{0} \ldots d_{n-1} \in \mathcal{D}_{1}$.

(b) We show that $\mathrm{o}(d[\iota])<\mathrm{o}(d)$ :

1. $d=\mathrm{Ax}_{\neg A, A}^{I}$ with $A \simeq \bigwedge\left(A_{\iota}\right)_{\iota \in J}: \mathrm{o}(d[\iota])=\max \left\{\omega^{\mathrm{rk}\left(A_{\iota}\right)} \cdot 2, \operatorname{lev}(\iota)\right\}+1<\omega^{I+\mathrm{rk}_{0}(A)+2}=\mathrm{o}(d)$.

2. $d=\mathrm{A} x_{\forall x \in a A}^{I}$ with $a \in \mathcal{T}_{I}: \mathrm{o}(d[b])<\max \left\{\omega^{\mathrm{rk}(b \in a)} \cdot 2, \omega^{\mathrm{rk}(b=b)} \cdot 2, \omega^{\mathrm{rk}(A(b))} \cdot 2\right\}+\omega<\omega^{I+\mathrm{rk}_{0}(A)+2}=\mathrm{o}(d)$.

3. $d=\mathrm{A} x_{\exists x \in a A}^{I}$ with $a \in \mathcal{T}_{I}: \mathrm{o}(d[b])<\omega^{\mathrm{rk}(b \in a)} \# \omega^{\mathrm{rk}(A(b))} \# \omega^{\mathrm{rk}(\exists x \in a A)}+\omega<\omega^{I+\mathrm{rk}_{0}(A)+2}=\mathrm{o}(d)$.

4. Otherwise: $\mathrm{o}(d[i])=\mathrm{o}\left(d_{i}\right)<\max \left\{\mathrm{o}\left(d_{0}\right), \ldots, \mathrm{o}\left(d_{n-1}\right)\right\}+1=\mathrm{o}(d)$.

(c) $\operatorname{tp}(d)=\mathrm{Cut}_{C} \Rightarrow \mathcal{J}=\mathrm{Cut}_{C} \Rightarrow \operatorname{rk}(C)<I+\operatorname{rk}_{0}(C) \leq \operatorname{deg}(d)$.

(d) $\operatorname{tp}(d)=\bigvee_{B}^{\iota} \Rightarrow \iota \in \mathcal{T}_{I} \cup\{0,1\} \Rightarrow \mathrm{k}(\iota)<I<\mathrm{o}(d)$.

(e) 1. If $d=\mathrm{Ax}_{\Delta}^{I}$ then $\mathrm{k}(\operatorname{tp}(d)) \subseteq \mathrm{k}(\operatorname{End}(d))=\mathrm{k}(d)$. Otherwise we have $\mathrm{k}(\operatorname{tp}(d))=\mathrm{k}(\mathcal{J}) \subseteq \mathrm{k}(d)$.

2. $\mathrm{o}(d)=\omega^{I+m}+n \in \mathcal{H}^{0}(\mathrm{k}(d))$.

(f) If $d[\iota]=d_{\iota}$ then $\mathrm{k}(d[\iota]) \subseteq \mathrm{k}(d)$ by definition. Otherwise the claim follows as in the proof of Theorem 2.1. (Actually for $d \in \mathcal{D}_{1}$ we have $\mathrm{k}(d[\iota]) \subseteq \mathrm{k}(d) \cup \mathrm{k}(\iota)$.)

\section{Theorem 3.6}

If the sequent $\left\{\phi_{1}, \ldots, \phi_{n}\right\}$ is logically valid then there is an $\operatorname{RS}^{1}$-derivation $d$ with $\operatorname{End}(d) \subseteq\left\{\phi_{1}^{I}, \ldots, \phi_{n}^{I}\right\}$, $\mathrm{FV}(d)=\mathrm{FV}(\operatorname{End}(d)), \mathrm{k}(d) \subseteq\{0, I\}$.

Proof:

Let PL be the Tait-style sequent calculus for 1st order predicate logic (without identity) in the language $\{\in, \mathrm{Ad}\}$. Given a PL-derivation of $\Gamma$ we define an $\mathrm{RS}^{1}$-derivation $d$ with $\operatorname{End}(d) \subseteq \Gamma^{I}$ and $\mathrm{k}(d) \subseteq\{I\}$.

Let us consider the $(\forall)$ - and $(\exists)$-rule:

1. Let $\forall x \phi \in \Gamma$, and $d_{0}$ be an $\operatorname{RS}^{1}$-derivation with $\operatorname{End}\left(d_{0}\right) \subseteq \Gamma^{I}, \phi_{x}^{I}(y)$ and $y \notin \mathrm{FV}(\Gamma)$.

1.1. $\phi \equiv x \in z \rightarrow \psi$ : Then $(\forall x \phi)^{I} \equiv \forall x \in z \psi^{I}$. Let $d:=\mathrm{Cut}_{\forall x \in \mathrm{L}_{I} \phi^{I}}\left(\forall_{\forall x \in \mathrm{L}_{I} \phi^{I}} d_{0}\right) \mathrm{Ax}_{\forall x \in z \psi^{I}}^{I}$.

1.2. otherwise: Then $(\forall x \phi)^{I} \equiv \forall x \in \mathrm{L}_{I} \phi^{I}$, and we set $d:=\forall_{\forall x \in \mathrm{L}_{I} \phi^{I}}^{y} d_{0}$.

2. Let $\exists x \phi \in \Gamma$, and $d_{0}$ be an $\mathrm{RS}^{1}$-derivation with $\operatorname{End}\left(d_{0}\right) \subseteq \Gamma^{I}, \phi_{x}^{I}(y)$.

2.1. $\phi \equiv x \in z \wedge \psi$ : Then $(\exists x \phi)^{I} \equiv \exists x \in z \psi^{I}$. Let $d:=\mathrm{Cut}_{\exists x \in \mathrm{L}_{I} \phi^{I}}\left(\exists_{\exists x \in \mathrm{L}_{I} \phi^{I}}^{y} d_{0}\right) \mathrm{Ax}_{\exists x \in z \psi^{I}}^{I}$.

2.2. otherwise: Then $(\exists x \phi)^{I} \equiv \exists x \in \mathrm{L}_{I} \phi^{I}$, and we set $d:=\exists_{\exists x \in \mathrm{L}_{I} \phi^{I}}^{y} d_{0}$. 
By Lemma 3.4 from $d$ we obtain an $\operatorname{RS}^{1}$-derivation $d^{\prime}$ with $\operatorname{End}\left(d^{\prime}\right) \subseteq \operatorname{End}(d), \operatorname{FV}\left(d^{\prime}\right)=\operatorname{FV}\left(\operatorname{End}\left(d^{\prime}\right)\right)$ and $\mathrm{k}\left(d^{\prime}\right) \subseteq \mathrm{k}(d) \cup\{0\} \subseteq\{0, I\}$.

Remark: For each $\mathrm{RS}^{1}$-derivation $d$ we have $\mathrm{o}(d)<\omega^{I+\omega}$ and $\operatorname{deg}(d)<I+\omega$.

\section{$\S 4$ The collapsing functions $\psi_{\kappa}$}

Definition (The Veblen hierarchy $\varphi$ )

$\varphi_{\alpha}:=$ ordering function of $\left\{\omega^{\beta}: \beta \in\right.$ On $\left.\& \forall \xi<\alpha\left(\varphi_{\xi}\left(\omega^{\beta}\right)=\omega^{\beta}\right)\right\}$

Corollary (Basic properties of $\varphi$ )

$(\varphi .1) \varphi_{0}(\beta)=\omega^{\beta}, \varphi_{1}(\beta)=\varepsilon_{\beta}$

$(\varphi .2) \xi, \eta<\varphi_{\alpha}(\beta) \Rightarrow \xi+\eta<\varphi_{\alpha}(\beta)$

$(\varphi .3) \beta_{0}<\beta \Rightarrow \varphi_{\alpha}\left(\beta_{0}\right)<\varphi_{\alpha}(\beta)$

$(\varphi .4) \quad \alpha_{0}<\alpha \Rightarrow \varphi_{\alpha_{0}}\left(\varphi_{\alpha}(\beta)\right)=\varphi_{\alpha}(\beta)$

\section{Definition}

$\widehat{\varphi}_{0}:=i d, \widehat{\varphi}_{\alpha}:=\varphi_{\alpha_{0}} \circ \ldots \circ \varphi_{\alpha_{n}}$, if $\alpha=\omega^{\alpha_{0}}+\ldots+\omega^{\alpha_{n}}$ with $\alpha_{0} \geq \ldots \geq \alpha_{n}$

For $\alpha \leq \beta$ let $\beta \dot{-\alpha}$ the unique ordinal $\xi$ such that $\alpha+\xi=\beta$.

\section{Lemma 4.1}

a) $\beta_{0}<\beta \Rightarrow \hat{\varphi}_{\alpha}\left(\beta_{0}\right)<\hat{\varphi}_{\alpha}(\beta)$,

b) $\hat{\varphi}_{\alpha+\beta}=\widehat{\varphi}_{\alpha} \circ \widehat{\varphi}_{\beta}$,

c) $\hat{\varphi}_{\gamma \cdot \alpha}=\hat{\varphi}_{\beta-\alpha} \circ \hat{\varphi}_{\gamma-\beta}$ if $\alpha \leq \beta \leq \gamma$.

Proof of b): Let $\alpha=\omega^{\alpha_{0}}+\ldots+\omega^{\alpha_{n}}, \beta=\omega^{\beta_{0}}+\ldots+\omega^{\beta_{m}}$ with $\alpha_{0} \geq \ldots \geq \alpha_{n}$ and $\beta_{0} \geq \ldots \geq \beta_{m}$. Then $\alpha+\beta=\omega^{\alpha_{0}}+\ldots+\omega^{\alpha_{k-1}}+\omega^{\beta_{0}}+\ldots+\omega^{\beta_{m}}$ with $k \leq n+1, \forall i<k\left(\alpha_{i} \geq \beta_{0}\right)$ and $\forall i\left(k \leq i \leq n \rightarrow \alpha_{i}<\beta_{0}\right)$.

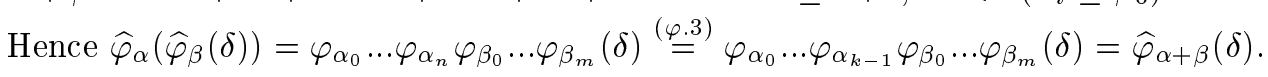

As before we use $\kappa, \pi, \tau$ to denote elements of $\mathrm{R}$. $\varphi$ denotes the function $(\alpha, \beta) \mapsto \varphi_{\alpha}(\beta)$, and $\boldsymbol{\Omega}$ denotes the function $\xi \mapsto \Omega_{\xi}$.

DEFINITION (The collapsing functions $\psi_{\kappa}$ )

By transfinite recursion on $\alpha$ we define ordinals $\psi_{\kappa} \alpha$ and sets $C(\alpha, \beta) \subseteq O n$ as follows

$\psi_{\kappa} \alpha:=\min \{\beta: \kappa \in C(\alpha, \beta) \& C(\alpha, \beta) \cap \kappa \subseteq \beta\}$

$C(\alpha, \beta):=$ the closure of $\beta \cup\{0, I\}$ under the functions $+, \varphi, \Omega,\left.\psi\right|_{\alpha}$,

where $\operatorname{dom}\left(\left.\psi\right|_{\alpha}\right):=\left\{(\pi, \xi): \xi<\alpha \& \pi \in \mathrm{R} \& \xi \in C\left(\xi, \psi_{\pi} \xi\right)\right\}$ and $\left.\psi\right|_{\alpha}(\pi, \xi):=\psi_{\pi} \xi$.

(Note that by I.H. $\psi_{\pi} \xi$ is already defined for all $\xi<\alpha, \pi \in$ R.)

We then set $\psi_{\kappa}: O n \longrightarrow O n, \psi_{\kappa}(\alpha):=\psi_{\kappa} \alpha$.

Abbreviation: $C_{\kappa}(\alpha):=C\left(\alpha, \psi_{\kappa} \alpha\right)$.

\section{Lemma 4.2}

a) $\beta<\pi \Rightarrow \operatorname{card}(C(\alpha, \beta))<\pi$ 
b) $C(\alpha, \beta)=\bigcup_{\eta<\beta} C(\alpha, \eta)$, for each limit ordinal $\beta$

c) $\kappa \in C(\alpha, \kappa)$

d) $C_{\kappa}(\alpha) \cap \kappa=\psi_{\kappa} \alpha$

Proof: cf. [Bu92]

\section{Lemma 4.3}

a) $\psi_{\kappa} \alpha<\kappa \& \psi_{\kappa} \alpha \notin C_{\kappa}(\alpha)$

b) $\alpha_{0}<\alpha \& \alpha_{0} \in C_{\kappa}\left(\alpha_{0}\right) \Rightarrow \psi_{\kappa} \alpha_{0}<\psi_{\kappa} \alpha$

c) $\psi_{\kappa} \alpha \notin\left\{\Omega_{\sigma}: \sigma<\Omega_{\sigma}\right\} \cup\{0\} \& \forall \xi, \eta<\psi_{\kappa} \alpha\left(\varphi_{\xi}(\eta)<\psi_{\kappa} \alpha\right)$

d) $\Omega_{\sigma} \in C(\alpha, \beta) \Rightarrow \sigma \in C(\alpha, \beta)$

e) $\omega^{\xi_{0}} \# \ldots \# \omega^{\xi_{n}} \in C(\alpha, \beta) \Rightarrow\left\{\xi_{0}, \ldots, \xi_{n}\right\} \subseteq C(\alpha, \beta)$

f) $\kappa=\Omega_{\sigma+1} \Rightarrow \Omega_{\sigma}<\psi_{\kappa} \alpha<\Omega_{\sigma+1}$

g) $\Omega_{\psi_{I} \alpha}=\psi_{I} \alpha$

h) $\Omega_{\sigma} \leq \gamma \leq \Omega_{\sigma+1} \& \gamma \in C(\alpha, \beta) \Rightarrow \sigma \in C(\alpha, \beta)$

i) $\alpha_{0} \leq \alpha \Rightarrow \psi_{\kappa} \alpha_{0} \leq \psi_{\kappa} \alpha \& C_{\kappa}\left(\alpha_{0}\right) \subseteq C_{\kappa}(\alpha)$

Proof: cf. [Bu92]. There is only one difference: In [Bu92] part b) of the lemma reads " $\alpha_{0}<\alpha \& \alpha_{0} \in$ $C_{\kappa}(\alpha) \Rightarrow \psi_{\kappa} \alpha_{0}<\psi_{\kappa} \alpha$ " which there is an immediate consequence of $4.2 \mathrm{~d}$ and $4.3 \mathrm{a}$ above. In the present version we also need $4.3 \mathrm{i}$ for proving $4.3 \mathrm{~b}$ :

$\alpha_{0}<\alpha \& \alpha_{0} \in C_{\kappa}\left(\alpha_{0}\right) \stackrel{4.3 \mathrm{i}+\text { Def }}{\Rightarrow} \alpha_{0}<\alpha \& \alpha_{0} \in C_{\kappa}\left(\alpha_{0}\right) \& \alpha_{0}, \kappa \in C_{\kappa}(\alpha) \stackrel{4.3 \mathrm{a}, 4.2 \mathrm{~d}}{\Rightarrow} \psi_{\kappa} \alpha_{0} \in C_{\kappa}(\alpha) \cap \kappa=\psi_{\kappa} \alpha$.

\section{Lemma 4.4}

a) $\psi_{\kappa_{0}} \alpha_{0}=\psi_{\kappa_{1}} \alpha_{1} \& \alpha_{i} \in C_{\kappa_{i}}\left(\alpha_{i}\right)$ for $i=0,1 \Longrightarrow \kappa_{0}=\kappa_{1} \& \alpha_{0}=\alpha_{1}$.

b) $\xi \in C_{\pi}(\xi) \& \psi_{\kappa} \alpha \leq \psi_{\pi} \xi \in C_{\kappa}(\alpha) \Longrightarrow \xi<\alpha \& \xi, \pi \in C_{\kappa}(\alpha)$.

Proof:

a) follows from L.4.3b,f,g.

b) From $\psi_{\kappa} \alpha \leq \psi_{\pi} \xi \in C_{\kappa}(\alpha)$ it follows (by L.4.3c) that $\psi_{\pi} \xi=\psi_{\tau} \eta$ with $\eta \in C_{\tau}(\eta) \& \eta<\alpha \& \tau, \eta \in C_{\kappa}(\alpha)$.

By $\xi \in C_{\pi}(\xi)$ and a) we obtain $\pi=\tau \& \xi=\eta$ and thus $\xi<\alpha \& \xi, \pi \in C_{\kappa}(\alpha)$.

Definition (The operators $\mathcal{H}_{\gamma}$ )

$\mathcal{H}_{\gamma}(X):=\bigcap\left\{C_{\kappa}(\alpha): X \subseteq C_{\kappa}(\alpha) \& \gamma<\alpha \& \kappa \in \mathrm{R}\right\} \quad(X \subseteq O n)$

where by convention $\mathcal{H}_{\gamma}(X):=O n$ if $\neg \exists \kappa, \alpha\left(X \subseteq C_{\kappa}(\alpha)\right)$.

\section{Lemma 4.5}

(H1) $\forall \alpha_{1}, \ldots, \alpha_{n}\left(\omega^{\alpha_{1}} \# \ldots \# \omega^{\alpha_{n}} \in \mathcal{H}_{\gamma}(X) \Leftrightarrow\left\{\alpha_{1}, \ldots, \alpha_{n}\right\} \subseteq \mathcal{H}_{\gamma}(X)\right)$,

(H2) $X \subseteq \mathcal{H}_{\gamma}(X)$,

(H3) $X^{\prime} \subseteq \mathcal{H}_{\gamma}(X) \Rightarrow \mathcal{H}_{\gamma}\left(X^{\prime}\right) \subseteq \mathcal{H}_{\gamma}(X)$,

(H4) $\mathcal{H}_{\gamma}(X)$ is closed under \#, $\lambda x . \omega \cdot x, \varphi, \Omega,\left(\xi \mapsto \xi^{+}\right)_{\xi<I}$,

(H5) $\xi \leq \gamma \& \xi, \pi \in \mathcal{H}_{\gamma}(X) \& \xi \in C_{\pi}(\xi) \Rightarrow \psi_{\pi} \xi \in \mathcal{H}_{\gamma}(X)$,

(H6) $\Omega_{\sigma} \leq \alpha \leq \Omega_{\sigma+1} \& \alpha \in \mathcal{H}_{\gamma}(X) \Rightarrow \Omega_{\sigma}, \Omega_{\sigma+1} \in \mathcal{H}_{\gamma}(X)$,

(H7) $\gamma<\delta \Rightarrow \mathcal{H}_{\gamma}(X) \subseteq \mathcal{H}_{\delta}(X)$ 


\section{Inductive definition of the set $\mathrm{OT}$ of ordinal terms}

(OT1) $0, I \in$ OT.

(OT2) $\omega^{\alpha_{0}}, \ldots, \omega^{\alpha_{n}} \in \mathrm{OT}(n \geq 1) \& \alpha_{n} \leq \ldots \leq \alpha_{0} \Longrightarrow \omega^{\alpha_{0}}+\ldots+\omega^{\alpha_{n}} \in \mathrm{OT}$.

(OT3) $\alpha, \beta \in$ OT \& $\alpha, \beta<\varphi_{\alpha}(\beta) \Longrightarrow \varphi_{\alpha}(\beta) \in$ OT.

(OT4) $\sigma \in \mathrm{OT} \& \sigma<\Omega_{\sigma} \Rightarrow \Omega_{\sigma} \in \mathrm{OT}$.

(OT5) $\alpha, \kappa \in \mathrm{OT} \& \alpha \in C_{\kappa}(\alpha) \Longrightarrow \psi_{\kappa} \alpha \in \mathrm{OT}$.

Remark From 4.3c and 4.4a it follows that the above inductive definition is deterministic, i.e., every element $\gamma \in$ OT can be generated in only one way by the rules (OT1)-(OT5). Consequently this definition associates with every $\gamma \in \mathrm{OT}$ a unique term $\operatorname{nf}(\gamma)$ (in the alphabet $\{0, I,+, \varphi, \Omega, \psi\}$ ), the normal form of $\gamma$. In the sequel $\gamma$ and $\operatorname{nf}(\gamma)$ are identified and called ordinal terms. Lemma 4.6 below is crucial for a primitive characterization of the set OT of ordinal terms. A primitive recursive characterization of the relation " $\alpha<\beta$ " for ordinal terms $\alpha, \beta \in \mathrm{OT}$ is easily obtained by means of Lemma 4.3b,c,f,g (for details cf. e.g. [Jä84] or [Bu86]). Finally, Lemma 4.8b shows that the relation " $\gamma \in \mathcal{H}_{\delta}(X)$ " (for $\gamma, \delta \in \mathrm{OT}$ and finite $X \subseteq \mathrm{OT})$ is primitive recursive.

Definition of $G_{\kappa} \alpha$ for $\alpha \in \mathrm{OT}$

$G_{\kappa} 0:=G_{\kappa} I:=\emptyset$

$G_{\kappa}(\xi \# \eta):=G_{\kappa} \xi \cup \ldots \cup G_{\kappa} \eta$

$G_{\kappa} \varphi_{\xi}(\eta):=G_{\kappa} \xi \cup G_{\kappa} \eta$, if $\xi, \eta<\varphi_{\xi}(\eta)$

$G_{\kappa} \Omega_{\xi}:=G_{\kappa} \xi$, if $\xi<\Omega_{\xi}$

$G_{\kappa} \psi_{\pi} \xi:= \begin{cases}G_{\kappa} \pi & \text { if } \pi<\kappa=I \\ \{\xi\} \cup G_{\kappa} \xi \cup G_{\kappa} \pi & \text { if } \kappa=\pi \text { or } \kappa<\psi_{\pi} \xi, \quad \text { if } \xi \in C_{\pi}(\xi) . \\ \emptyset & \text { otherwise }\end{cases}$

Lemma $4.6 \gamma \in \mathrm{OT} \Longrightarrow\left(\gamma \in C_{\kappa}(\alpha) \Leftrightarrow G_{\kappa} \gamma \subseteq \alpha\right)$

Proof: Let $\gamma=\psi_{\pi} \xi$ with $\xi \in C_{\pi}(\xi)$. So $\gamma<\pi \leq I$.

1. $\pi<\kappa=I: \gamma \in C_{I}(\alpha) \Leftrightarrow \gamma<\psi_{I} \alpha \Leftrightarrow \pi<\psi_{I} \alpha \Leftrightarrow \pi \in C_{I}(\alpha)$.

2. $\kappa=\pi: \gamma \in C_{\kappa}(\alpha) \Leftrightarrow \psi_{\kappa} \xi<\psi_{\kappa} \alpha \Leftrightarrow \xi<\alpha \Leftrightarrow \xi<\alpha \& \xi, \pi \in C_{\kappa}(\alpha)$.

3. $\kappa<\psi_{\pi} \xi: \gamma \in C_{\kappa}(\alpha) \Leftrightarrow \xi<\alpha \& \xi, \pi \in C_{\kappa}(\alpha)$.

4. Otherwise: Then $\pi \neq \kappa \& \psi_{\pi} \xi<\kappa<I$ which yields $\psi_{\pi} \xi<\psi_{\kappa} \alpha$.

Definition of $Y(\alpha)$

$Y(0):=Y(I):=\{0, I\}$,

$Y(\xi \# \eta):=Y(\xi) \cup Y(\eta)$,

$Y\left(\varphi_{\xi}(\eta)\right):=Y(\xi) \cup Y(\eta)$ if $\xi, \eta<\varphi_{\xi}(\eta)$,

$Y\left(\Omega_{\xi}\right):=\left\{\Omega_{\xi}\right\} \cup Y(\xi)$ if $0<\xi<\Omega_{\xi}$,

$Y\left(\psi_{\pi} \xi\right):=\left\{\begin{array}{ll}Y(\pi) \cup Y(\xi) & \text { if } \pi<I \\ \left\{\psi_{\pi}(\xi)\right\} \cup Y(\xi) & \text { if } \pi=I\end{array}, \quad\right.$ if $\xi \in C_{\pi}(\xi)$ 
Lemma $4.7 \gamma \in$ OT \& $\kappa<\tau<I \&\left[\kappa, \tau\left[\cap Y(\gamma)=\emptyset \Rightarrow G_{\kappa} \gamma=G_{\tau} \gamma\right.\right.$

Proof by induction on the definition of $G_{\kappa} \gamma$ : Let $\gamma=\psi_{\pi} \xi$ with $\xi \in C_{\pi}(\xi)$.

1. $\pi<\kappa=I$ : impossible.

2. $\kappa=\pi$ : impossible, since $\pi \in Y(\gamma)$ if $\pi<I$.

3. $\kappa<\psi_{\pi} \xi$ : We have $G_{\kappa} \gamma=\{\xi\} \cup G_{\kappa} \xi \cup G_{\kappa} \pi \stackrel{\text { IH }}{=}\{\xi\} \cup G_{\tau} \xi \cup G_{\tau} \pi$. It remains to prove $\tau=\pi$ or $\tau<\psi_{\pi} \xi$.

3.1. $\pi \neq \tau \& \pi<I$ : Then $\psi_{\pi} \xi \leq \tau$ would imply $\pi<\tau$, and thus $\pi \in[\kappa, \tau[\cap Y(\gamma)$. Contradiction.

3.2. $\tau<\pi=I$ : Then $\psi_{\pi} \xi \leq \tau$ would imply $\psi_{\pi} \xi \in[\kappa, \tau[\cap Y(\gamma)$. Contradiction.

4. $\pi \neq \kappa \& \psi_{\pi} \xi<\kappa<I$ : Then $\psi_{\pi} \xi<\kappa<\tau<I$ and thus $\pi \neq \tau$. Hence $G_{\kappa} \gamma=\emptyset=G_{\tau} \gamma$.

\section{Lemma 4.8}

Let $X \subseteq$ OT finite, $Y_{\gamma, X}:=Y(\gamma) \cup \bigcup_{\xi \in X} Y(\xi), Y_{\gamma, X}^{+}:=Y_{\gamma, X} \cup\left\{\rho^{+}: \rho \in Y_{\gamma, X} \cap I\right\}$, $G_{\kappa}(X):=\bigcup_{\xi \in X} G_{\kappa} \xi, \mathrm{m}(\delta, \kappa, X):=\max \left(\{\delta\} \cup G_{\kappa}(X)\right)+1$.

a) $\forall \kappa \in Y_{\gamma, X}^{+}\left(G_{\kappa} \gamma \subseteq \mathrm{m}(\delta, \kappa, X)\right) \Longrightarrow \forall \tau\left(G_{\tau} \gamma \subseteq \mathrm{m}(\delta, \tau, X)\right)$.

b) $\gamma \in \mathcal{H}_{\delta}(X) \Longleftrightarrow \forall \kappa \in Y_{\gamma, X}^{+}\left(G_{\kappa} \gamma \subseteq \mathrm{m}(\delta, \kappa, X)\right)$.

Proof:

a) Let $\tau \notin Y_{\gamma, X}^{+}$and $\kappa:=\max \left\{\kappa^{\prime} \in Y_{\gamma, X}^{+}: \kappa^{\prime}<\tau\right\}$ (note that $Y_{\gamma, X}^{+}$is finite!). Then $\left[\kappa, \tau\left[\cap Y_{\gamma, X}=\emptyset\right.\right.$ and therefore $G_{\tau} \gamma=G_{\kappa} \gamma \subseteq \mathrm{m}(\delta, \kappa, X)=\mathrm{m}(\delta, \tau, X)$.

b) $\gamma \in \mathcal{H}_{\delta}(X) \Longleftrightarrow \forall \alpha>\delta \forall \kappa\left(X \subseteq C_{\kappa}(\alpha) \Rightarrow \gamma \in C_{\kappa}(\alpha)\right) \Longleftrightarrow \forall \kappa \forall \alpha>\delta\left(G_{\kappa}(X) \subseteq \alpha \Rightarrow G_{\kappa} \gamma \subseteq \alpha\right) \Longleftrightarrow$ $\forall \kappa\left(G_{\kappa} \gamma \subseteq \mathrm{m}(\delta, \kappa, X)\right) \Longleftrightarrow \forall \kappa \in Y_{\gamma, X}^{+}\left(G_{\kappa} \gamma \subseteq \mathrm{m}(\delta, \kappa, X)\right)$

\section{$\S 5$ The notation systems $\mathbf{H}_{\delta}$}

In this section we introduce a family $\left(\mathbf{H}_{\gamma}\right)_{\gamma \in O n}$ of notation systems (for $\mathrm{RS}^{\infty}$-derivations) extending $\mathbf{R S}^{+}$. $\mathbf{H}_{\gamma}$ contains notations for $\mathrm{RS}^{\infty}$-derivations which are $\mathcal{H}_{\gamma}$-controlled (in the sense of [Bu92]) and arise from $\mathrm{RS}^{\infty}$-derivations representable in $\mathbf{R S}^{+}$by application of the cut-elimination and collapsing procedures described in (the proofs of $3.14,3.16,3.17,4.8$ of) [Bu92]. From now on $A, B, C, D$ always denote RS-sentences.

Definition $\bar{K}:=\left\{\bar{\Omega}_{\sigma}: \sigma \leq I\right\}$ with $\bar{\Omega}_{\sigma}:= \begin{cases}\Omega_{\sigma}+1 & \text { if } \Omega_{\sigma} \in \mathrm{R} \\ \Omega_{\sigma} & \text { otherwise }\end{cases}$

We introduce the following new inferences:

$\left(\mathrm{R}_{C}\right) \frac{C \quad \neg C}{\emptyset}$ if $\operatorname{rk}(C) \notin \mathrm{R}$,

$\left(\mathrm{E}_{\rho}^{\sigma}\right) \frac{\emptyset}{\emptyset}$ if $\rho \leq \sigma$ and $[\rho, \sigma[\cap \mathrm{R}=\emptyset$,

$\left(\mathrm{B}_{C}^{\beta, \kappa}\right) \frac{C}{C^{(\beta, \kappa)}}$ if $C \in \Sigma(\kappa) \cup \Pi(\kappa)$ and $\beta<\kappa$,

$\left(\operatorname{Col}_{\gamma}^{\mu, \kappa}\right) \frac{\emptyset}{\emptyset}$ if $\mu \in \bar{K} \& \gamma, \mu, \kappa \in C_{\kappa}^{*}(\gamma+1)$

$\mathrm{k}\left(\mathrm{R}_{C}\right):=\emptyset, \mathrm{k}\left(\mathrm{E}_{\rho}^{\sigma}\right):=\{\sigma\}, \mathrm{k}\left(\mathrm{B}_{C}^{\beta, \kappa}\right):=\{\beta\}, \mathrm{k}\left(\mathrm{Col}_{\gamma}^{\mu, \kappa}\right):=\{\mu, \kappa, \gamma\}$ 


\section{Inductive Definition of a set $\mathcal{D}^{*}$ of derivations}

1. $\mathcal{D}^{+} \subseteq \mathcal{D}^{*}$, i.e., every $\mathrm{RS}^{0}$-derivation and every closed $\mathrm{RS}^{1}$-derivation belongs to $\mathcal{D}^{*}$.

2. $h_{0}, h_{1} \in \mathcal{D}^{*} \Rightarrow \mathrm{R}_{C} h_{0} h_{1}, \mathrm{E}_{\rho}^{\sigma} h_{0}, \mathrm{~B}_{C}^{\beta, \kappa} h_{0}, \operatorname{Col}_{\gamma}^{\mu, \kappa} h_{0} \in \mathcal{D}^{*}$.

In the following $h, h_{i}$ always denote elements of $\mathcal{D}^{*}$.

Now we are going to define $\mathrm{o}(h), \operatorname{deg}(h), \operatorname{tp}(h), h[\iota]$ by recursion on the build up of $h$. For $h \in \mathcal{D}^{+}$these items have already been defined in $\S 1, \S 2$. So it remains to consider $\mathrm{R}_{C} h_{0} h_{1}, \mathrm{E}_{\rho}^{\sigma} h_{0}, \mathrm{~B}_{C}^{\beta, \kappa} h_{0}$, Col ${ }_{\gamma}^{\mu, \kappa} h_{0}$.

Definition of $\mathrm{o}(h)$ and $\operatorname{deg}(h)$

$$
\begin{array}{ll}
\mathrm{o}\left(\mathrm{R}_{C} h_{0} h_{1}\right):=\mathrm{o}\left(h_{0}\right) \# \mathrm{o}\left(h_{1}\right) & , \operatorname{deg}\left(\mathrm{R}_{C} h_{0} h_{1}\right):=\max \left\{\operatorname{rk}(C), \operatorname{deg}\left(h_{0}\right), \operatorname{deg}\left(h_{1}\right)\right\} \\
\mathrm{o}\left(\mathrm{E}_{\rho}^{\sigma} h_{0}\right):=\widehat{\varphi}_{\sigma-\rho}\left(\mathrm{o}\left(h_{0}\right)\right) & , \operatorname{deg}\left(\mathrm{E}_{\rho}^{\sigma} h_{0}\right):=\rho \\
\mathrm{o}\left(\mathrm{B}_{C}^{\beta, \kappa} h_{0}\right):=\mathrm{o}\left(h_{0}\right) & , \operatorname{deg}\left(\mathrm{B}_{C}^{\beta, \kappa} h_{0}\right):=\operatorname{deg}\left(h_{0}\right) \\
\mathrm{o}\left(\mathrm{Col}_{\gamma}^{\mu, \kappa} h_{0}\right):=\psi_{\kappa}\left(\gamma+\omega^{\mu+\mathrm{o}\left(h_{0}\right)}\right) & , \operatorname{deg}\left(\mathrm{Col}_{\gamma}^{\mu, \kappa} h_{0}\right):=\psi_{\kappa}\left(\gamma+\omega^{\mu+\mathrm{o}\left(h_{0}\right)}\right) .
\end{array}
$$

Definition of $\operatorname{tp}(h)$ and $h[\iota](\iota \in|\operatorname{tp}(h)|)$

1. $h=\mathrm{R}_{C} h_{0} h_{1}$ with $\operatorname{rk}(C) \notin \mathrm{R}$ :

Since $\operatorname{rk}(C) \notin \mathrm{R}$, we have $\left(C \in \Delta\left(\operatorname{tp}\left(h_{i}\right)\right)\right.$ or $\left.\neg C \in \Delta\left(\operatorname{tp}\left(h_{i}\right)\right) \Rightarrow \operatorname{tp}\left(h_{i}\right) \neq \operatorname{Ref}_{A}^{\kappa}\right)$.

1.1. $C \notin \Delta\left(\operatorname{tp}\left(h_{0}\right)\right): \operatorname{tp}(h):=\operatorname{tp}\left(h_{0}\right), h[\iota]:=\mathrm{R}_{C} h_{0}[\iota] h_{1}$.

1.2. $\neg C \notin \Delta\left(\operatorname{tp}\left(h_{1}\right)\right): \operatorname{tp}(h):=\operatorname{tp}\left(h_{1}\right), h[\iota]:=\mathrm{R}_{C} h_{0} h_{1}[\iota]$.

1.3. $C \in \Delta\left(\operatorname{tp}\left(h_{0}\right)\right)$ and $\neg C \in \Delta\left(\operatorname{tp}\left(h_{1}\right)\right)$ :

1.3.1. $C \simeq \bigwedge\left(C_{\iota}\right)_{\iota \in J}:$ Then $\operatorname{tp}\left(h_{0}\right)=\bigwedge_{C}$, and $\operatorname{tp}\left(h_{1}\right)=\bigvee_{\neg C}^{\iota_{0}}$ with $\iota_{0} \in J$. $\operatorname{tp}(h):=$ Cut $_{C_{\iota_{0}}}, h[0]:=\mathrm{R}_{C} h_{0}\left[\iota_{0}\right] h_{1}, h[1]:=\mathrm{R}_{C} h_{0} h_{1}[0]$.

1.3.2. $C \simeq \bigvee\left(C_{\iota}\right)_{\iota \in J}:$ analogous to 1.3 .1

2. $h=\mathrm{E}_{\rho}^{\sigma} h_{0}$ :

2.1. $\operatorname{tp}\left(h_{0}\right)=$ Cut $_{C}$ with $\rho \leq \nu:=\operatorname{rk}(C)<\sigma: \operatorname{tp}(h):=\operatorname{Rep}, h[0]:=\mathrm{E}_{\rho}^{\nu} \mathrm{R}_{C} \mathrm{E}_{\nu}^{\sigma} h_{0}[0] \mathrm{E}_{\nu}^{\sigma} h_{0}[1]$,

2.2. otherwise: $\operatorname{tp}(h):=\operatorname{tp}\left(h_{0}\right), h[\iota]:=\mathrm{E}_{\rho}^{\sigma} h_{0}[\iota]$.

3. $h=\mathrm{B}_{C}^{\beta, \kappa} h_{0}$ with $C \in \Sigma(\kappa) \cup \Pi(\kappa)$ and $\beta<\kappa$ :

3.1. $C \notin \Delta\left(\operatorname{tp}\left(h_{0}\right)\right): \operatorname{tp}(h):=\operatorname{tp}\left(h_{0}\right)$ and $h[\iota]:=\mathrm{B}_{C}^{\beta, \kappa} h_{0}[\iota]$.

3.2. $C \in \Delta\left(\operatorname{tp}\left(h_{0}\right)\right)$ :

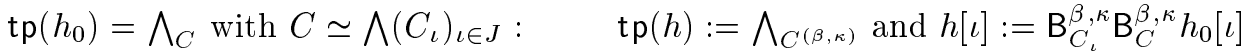
$\operatorname{tp}\left(h_{0}\right)=\bigvee_{C}^{\iota_{0}}$ with $C \simeq \bigvee\left(C_{\iota}\right)_{\iota \in J}: \quad \operatorname{tp}(h):=\bigvee_{C^{(\beta, \kappa)}}^{\iota_{0}}$ and $h[0]:=\mathrm{B}_{C_{\iota_{0}}^{\beta, \kappa}} \mathrm{B}_{C}^{\beta, \kappa} h_{0}[0]$, $\operatorname{tp}\left(h_{0}\right)=\operatorname{Ref}_{A}^{\kappa}$ with $C \equiv \exists z \in \mathrm{L}_{\kappa} A^{(z, \kappa)}: \operatorname{tp}(h):=\bigvee_{C^{(\beta, \kappa)}}^{\mathrm{L}_{\alpha_{0}}}$ and $h[0]:=\mathrm{B}_{A}^{\alpha_{0}, \kappa} \mathrm{B}_{C}^{\beta, \kappa} h_{0}[0]$ with $\alpha_{0}:=\min \left\{\mathrm{o}\left(h_{0}[0]\right), \beta\right\}$.

4. $\quad h=\operatorname{Col}_{\gamma}^{\mu, \kappa} h_{0}$ :

4.1. $\operatorname{tp}\left(h_{0}\right)=$ Cut $_{C}$ with $\kappa \leq \operatorname{rk}(C)<\mu$ and $\operatorname{rk}(C)=\pi \in \operatorname{R}: \operatorname{tp}(h):=\operatorname{Rep}$.

W.l.o.g.: $C=\exists x \in \mathrm{L}_{\pi} A(x) \in \Sigma(\pi)$

$\alpha_{i}:=\mathrm{o}\left(h_{0}[i]\right), \gamma_{0}:=\gamma+\omega^{\mu+\alpha_{0}}, \gamma_{1}:=\gamma_{0}+\omega^{\mu+\alpha_{1}}, \beta:=\psi_{\pi}\left(\gamma_{0}\right), \sigma:=\psi_{\pi}\left(\gamma_{1}\right)$ 


$$
\begin{aligned}
& \mu^{\prime}:= \begin{cases}\bar{\Omega}_{\nu} & \text { if } \pi=\Omega_{\nu+1}<I \\
\sigma & \text { if } \pi=I\end{cases} \\
& h[0]:=\operatorname{Col}_{\gamma_{1}}^{\mu^{\prime},{ }^{\prime}} \mathrm{E}_{\mu^{\prime}}^{\sigma} \mathrm{R}_{C(\beta, \pi)}\left(\mathrm{B}_{C}^{\beta, \pi} \operatorname{Col}_{\gamma}^{\mu, \pi} h_{0}[0]\right)\left(\operatorname{Col}_{\gamma_{0}}^{\mu, \pi} \mathrm{B}_{\neg C}^{\beta, \pi} h_{0}[1]\right)=\frac{\frac{\frac{h_{0}[0]}{\mathrm{Col}_{\gamma}^{\mu, \pi}}}{\mathrm{B}_{C}^{\beta, \pi}} \quad \frac{\frac{h_{0}[1]}{\mathrm{B}_{\rightarrow C}^{\beta, \pi}}}{\operatorname{Co}_{\gamma_{0}}^{\mu, \pi}}}{\frac{\mathrm{R}_{C^{(\beta, \pi)}}}{\frac{\mathrm{E}_{\mu^{\prime}}^{\sigma}}{\mathrm{Co}_{\gamma_{1}}^{\mu^{\prime}, \kappa}}}}
\end{aligned}
$$

4.2. $\operatorname{tp}\left(h_{0}\right)=$ Cut $_{C}$ with $\kappa \leq \operatorname{rk}(C)<\mu$ and $\operatorname{rk}(C) \notin \mathrm{R}$ :

Let $\pi:=\operatorname{rk}(C)^{+}$.

Since $\operatorname{rk}(C)<\mu \leq I+1$ and $\operatorname{rk}(C) \neq I$, we have $\pi<I$ and thus $\pi=\Omega_{\nu+1}$ for some $\nu$. $\alpha_{i}:=\mathrm{o}\left(h_{0}[i]\right), \gamma_{0}:=\gamma+\omega^{\mu+\alpha_{0}}, \gamma_{1}:=\gamma+\omega^{\mu+\alpha_{1}}, \sigma:=\max \left\{\psi_{\pi}\left(\gamma_{0}\right), \psi_{\pi}\left(\gamma_{1}\right)\right\}, \mu^{\prime}:=\bar{\Omega}_{\nu}$, $\gamma^{\prime}:=\max \left\{\gamma_{0}, \gamma_{1}\right\}$.

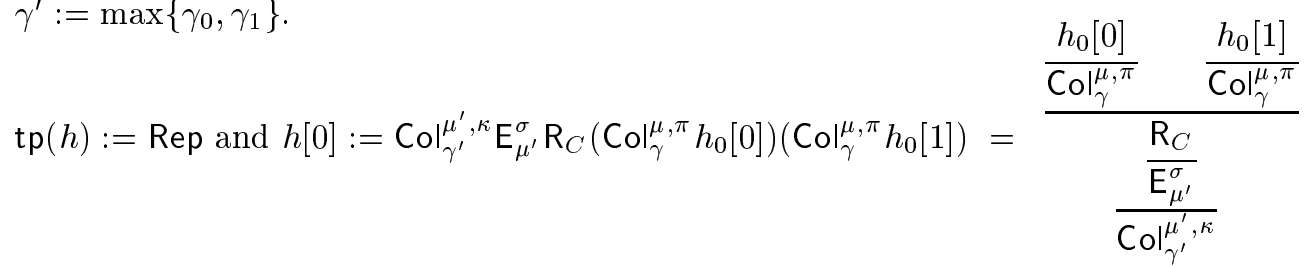

4.3. Otherwise: $\operatorname{tp}(h):=\operatorname{tp}\left(h_{0}\right), h[\iota]:=\operatorname{Col}_{\gamma}^{\mu, \kappa} h_{0}[\iota]$.

Abbreviation: $C_{\kappa}^{*}(\delta):=\bigcap_{\tau \geq \kappa} C_{\tau}(\delta)\left[=C_{\kappa}(\delta) \cap C_{I}(\delta)\right]$

\section{Inductive Definition of $\mathbf{H}_{\delta}$}

0. $h \in \mathcal{D}^{+} \Rightarrow h \in \mathbf{H}_{\delta}$.

1. $h_{0}, h_{1} \in \mathbf{H}_{\delta} \& \operatorname{rk}(C) \notin \mathrm{R} \Longrightarrow \mathrm{R}_{C} h_{0} h_{1} \in \mathbf{H}_{\delta}$.

2. $h_{0} \in \mathbf{H}_{\delta} \& \rho \leq \sigma \&\left[\rho, \sigma\left[\cap \mathrm{R}=\emptyset \& \operatorname{deg}\left(h_{0}\right) \leq \sigma \Longrightarrow \mathbf{E}_{\rho}^{\sigma} h_{0} \in \mathbf{H}_{\delta}\right.\right.$.

3. $h_{0} \in \mathbf{H}_{\delta} \& \beta<\kappa \& C \in \Sigma(\kappa) \cup \Pi(\kappa) \&\left(C \notin \Pi(\kappa) \Rightarrow \mathrm{o}\left(h_{0}\right) \leq \beta\right) \Longrightarrow \mathrm{B}_{C}^{\beta, \kappa} h_{0} \in \mathbf{H}_{\delta}$.

4. $\left.\begin{array}{l}h_{0} \in \mathbf{H}_{\gamma} \& \mu \in \bar{K} \& \mathrm{k}\left(h_{0}\right) \cup\{\mu, \kappa, \gamma\} \subseteq C_{\kappa}^{*}(\gamma+1) \\ \operatorname{End}\left(h_{0}\right) \subseteq \Sigma(\kappa) \& \operatorname{deg}\left(h_{0}\right) \leq \mu \& \gamma+\omega^{\mu+\mathrm{o}\left(h_{0}\right)} \leq \delta\end{array}\right\} \Longrightarrow \operatorname{Col}_{\gamma}^{\mu, \kappa} h_{0} \in \mathbf{H}_{\delta}$.

Corollary $\delta \leq \delta^{\prime} \Rightarrow \mathbf{H}_{\delta} \subseteq \mathbf{H}_{\delta^{\prime}}$.

Lemma 5.1 $h \in \mathbf{H}_{\delta} \Rightarrow \mathrm{o}(h) \in \mathcal{H}_{\delta}(\mathbf{k}(h))$.

Proof by induction on the build up of $h$ :

0. $h \in \mathcal{D}^{+} \stackrel{\text { Th..3.5 }}{\Rightarrow} \mathrm{o}(h) \in \mathcal{H}^{0}(\mathrm{k}(h)) \subseteq \mathcal{H}_{\delta}(\mathrm{k}(h))$.

1. $\mathrm{IH} \Rightarrow \mathrm{o}\left(h_{i}\right) \in \mathcal{H}_{\delta}\left(\mathrm{k}\left(h_{i}\right)\right) \Rightarrow \mathrm{o}(h)=\mathrm{o}\left(h_{0}\right) \# \mathrm{o}\left(h_{1}\right) \in \mathcal{H}_{\delta}(\mathrm{k}(h))$.

2. $\mathrm{o}\left(h_{0}\right) \stackrel{\mathrm{IH}}{\in} \mathcal{H}_{\delta}\left(\mathrm{k}\left(h_{0}\right)\right) \Rightarrow \mathrm{o}(h)=\widehat{\varphi}_{\sigma-\rho}\left(\mathrm{o}\left(h_{0}\right)\right) \in \mathcal{H}_{\delta}\left(\mathrm{k}\left(h_{0}\right) \cup\{\sigma\}\right)=\mathcal{H}_{\delta}(\mathrm{k}(h))$.

3. $\mathrm{o}(h)=\mathrm{o}\left(h_{0}\right) \stackrel{\mathrm{IH}}{\in} \mathcal{H}_{\delta}\left(\mathrm{k}\left(h_{0}\right)\right) \subseteq \mathcal{H}_{\delta}(\mathrm{k}(h))$.

4. o $\left(h_{0}\right) \stackrel{\mathrm{IH}}{\in} \mathcal{H}_{\gamma}\left(\mathrm{k}\left(h_{0}\right)\right) \subseteq C_{\kappa}(\gamma+1) \&\{\mu, \gamma\} \subseteq C_{\kappa}(\gamma+1) \& \gamma \leq \delta \Rightarrow$ $\gamma+\omega^{\mu+\mathrm{o}\left(h_{0}\right)}, \kappa \in \mathcal{H}_{\delta}\left(\mathrm{k}\left(h_{0}\right) \cup\{\mu, \kappa, \gamma\}\right)=\mathcal{H}_{\delta}(\mathrm{k}(h)) \& \gamma+\omega^{\mu+\mathrm{o}\left(h_{0}\right)} \in C_{\kappa}\left(\gamma+\omega^{\mu+\mathrm{o}\left(h_{0}\right)}\right) \Rightarrow$ $\mathrm{o}(h)=\psi_{\kappa}\left(\gamma+\omega^{\mu+\mathrm{o}\left(h_{0}\right)}\right) \in \mathcal{H}_{\delta}(\mathbf{k}(h))$, since $\gamma+\omega^{\mu+\mathrm{o}\left(h_{0}\right)} \leq \delta$. 


\section{Theorem 5.2}

$\left(\mathbf{H}_{\delta}, \mathrm{o}, \mathrm{deg}, \mathrm{tp},[]\right)$ is a notation system for $\mathrm{RS}^{\infty}$-derivation, and it is controlled by $\mathcal{H}_{\delta}$.

This means that $\mathbf{H}_{\delta} \ni h \vdash_{\rho}^{\alpha} \Gamma$ (and $\left.\mathcal{I}:=\operatorname{tp}(h)\right)$ implies

(a) $\Delta(\mathcal{I}) \subseteq \Gamma$,

(b) $\mathbf{H}_{\delta} \ni h[\iota] \vdash_{\rho}^{\alpha_{\iota}} \Gamma, \Delta_{\iota}(\mathcal{I})$ with $\alpha_{\iota}<\alpha \quad(\iota \in|\mathcal{I}|)$,

(c) $\mathcal{I}=\mathrm{Cut}_{C} \Rightarrow \operatorname{rk}(C)<\rho$,

(d) $\mathcal{I}=\bigvee_{A}^{\iota_{0}} \Rightarrow \mathrm{k}\left(\iota_{0}\right)<\alpha$,

(e) $\mathrm{k}(\mathcal{I}) \cup\{\alpha\} \subseteq \mathcal{H}_{\delta}(\mathrm{k}(h))$,

(f) $\mathrm{k}(h[\iota]) \subseteq \mathcal{H}_{\delta}(\mathrm{k}(h) \cup \mathrm{k}(\iota))$ for each $\iota \in|\mathcal{I}|$.

Proof by induction on the build up of $h$ :

The proof is very long, since we have treated all cases and carried out all details. Large parts of the proof are just routine verifications.

Assume $\mathbf{H}_{\delta} \ni h \vdash_{\rho}^{\alpha} \Gamma$.

We abbreviate $\mathbf{H}_{\delta} \ni h^{\prime} \vdash_{\rho^{\prime}}^{\alpha^{\prime}} \Gamma^{\prime}$ by $h^{\prime} H_{\rho^{\prime}}^{\alpha^{\prime}} \Gamma^{\prime}$.

0. $h \in \mathcal{D}^{+}$: The claim follows from Theorem 3.5 and the fact that $\mathcal{H}^{0}(X) \subseteq \mathcal{H}_{\delta}(X)$ for all $X \subseteq O n, \delta \in O n$.

1. $h=\mathrm{R}_{C} h_{0} h_{1}$ with $\operatorname{rk}(C) \notin \mathrm{R}$ :

Then $h_{0} \uplus_{\rho}^{\beta} \Gamma, C$ and $h_{1} \uplus_{\rho}^{\gamma} \Gamma, \neg C$ with $\alpha=\beta \# \gamma$ and $\operatorname{rk}(C) \leq \rho$.

1.1. $C \notin \Delta\left(\operatorname{tp}\left(h_{0}\right)\right)$ : Then $\mathcal{I}=\operatorname{tp}\left(h_{0}\right)$ and $h[\iota]=\mathrm{R}_{C} h_{0}[\iota] h_{1}$.

(a) $\Delta(\mathcal{I})=\Delta\left(\operatorname{tp}\left(h_{0}\right)\right) \stackrel{\mathrm{IH}}{\subseteq} \operatorname{End}\left(h_{0}\right) \backslash\{C\} \subseteq \operatorname{End}(h)$.

(b) By IH $h_{0}[\iota] H_{\rho}^{\beta_{\iota}} \Gamma, C, \Delta_{\iota}(\mathcal{I})$ with $\beta_{\iota}<\beta$. Hence $h[\iota] H_{\rho}^{\beta_{\iota} \# \gamma} \Gamma, \Delta_{\iota}(\mathcal{I})$ with $\beta_{\iota} \# \gamma<\beta \# \gamma=\alpha$.

(c) $\mathcal{I}=\mathrm{Cut}_{A} \Rightarrow \operatorname{tp}\left(h_{0}\right)=\mathrm{Cut}_{A} \stackrel{\mathrm{IH}}{\Rightarrow} \operatorname{rk}(A)<\rho$.

(d) $\mathcal{I}=\bigvee_{A}^{\iota_{0}} \Rightarrow \operatorname{tp}\left(h_{0}\right)=\bigvee_{A}^{\iota_{0}} \stackrel{\text { IH }}{\Rightarrow} \mathrm{k}\left(\iota_{0}\right)<\mathrm{o}\left(h_{0}\right) \leq \mathrm{o}(h)$.

(e) $\mathrm{k}(\mathcal{I})=\mathrm{k}\left(\mathrm{tp}\left(h_{0}\right)\right) \stackrel{\mathrm{IH}}{\subseteq} \mathcal{H}_{\delta}\left(\mathrm{k}\left(h_{0}\right)\right) \subseteq \mathcal{H}_{\delta}(\mathrm{k}(h))$.

(f) $\mathrm{k}(h[\iota])=\mathrm{k}\left(h_{0}[\iota]\right) \cup \mathrm{k}\left(h_{1}\right) \stackrel{\mathrm{IH}}{\subseteq} \mathcal{H}_{\delta}\left(\mathrm{k}\left(h_{0}\right) \cup \mathrm{k}(\iota)\right) \cup \mathrm{k}\left(h_{1}\right) \subseteq \mathcal{H}_{\delta}\left(\mathrm{k}\left(h_{0}\right) \cup \mathrm{k}\left(h_{1}\right) \cup \mathrm{k}(\iota)\right)=\mathcal{H}_{\delta}(\mathrm{k}(h) \cup \mathrm{k}(\iota))$.

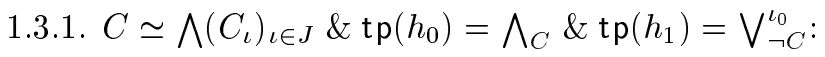

Then $\mathcal{I}=$ Cut $_{C_{\iota_{0}}}$ and $h[0]=\mathrm{R}_{C} h_{0}\left[\iota_{0}\right] h_{1}, h[1]=\mathrm{R}_{C} h_{0} h_{1}[0]$.

(a) $\Delta(\mathcal{I})=\emptyset$.

(b) By IH $h_{0}\left[\iota_{0}\right] H_{\rho}^{\beta_{\iota_{0}}} \Gamma, C, C_{\iota_{0}}$ and $h_{1}[0] H_{\rho}^{\gamma_{0}} \Gamma, \neg C, \neg C_{\iota_{0}}$ with $\beta_{\iota_{0}}<\beta$ and $\gamma_{0}<\gamma$.

Hence $h[0] H_{\rho}^{\beta_{\iota_{0}} \# \gamma} \Gamma, C, C_{\iota_{0}}$ with $\beta_{\iota_{0}} \# \gamma<\alpha$, and $h[1] H_{\rho}^{\beta \# \gamma_{0}} \Gamma, \neg C_{\iota_{0}}$ with $\beta \# \gamma_{0}<\alpha$.

(c) $\operatorname{rk}\left(C_{\iota_{0}}\right)<\operatorname{rk}(C) \leq \rho$.

(e) $\mathrm{k}(\mathcal{I})=\mathrm{k}\left(C_{\iota_{0}}\right) \subseteq \mathrm{k}(C) \cup \mathrm{k}\left(\iota_{0}\right)=\mathrm{k}\left(\mathrm{tp}\left(h_{1}\right)\right) \stackrel{\mathrm{IH}}{\subseteq} \mathcal{H}_{\delta}\left(\mathrm{k}\left(h_{1}\right)\right) \subseteq \mathcal{H}_{\delta}(\mathrm{k}(h))$.

(f) $\mathrm{k}(h[0])=\mathrm{k}\left(h_{0}\left[\iota_{0}\right]\right) \cup \mathrm{k}\left(h_{1}\right) \stackrel{\mathrm{IH}}{\subseteq} \mathcal{H}_{\delta}\left(\mathrm{k}\left(h_{0}\right) \cup \mathrm{k}\left(\iota_{0}\right)\right) \cup \mathrm{k}\left(h_{1}\right)$.

Hence $\mathrm{k}(h[0]) \subseteq \mathcal{H}_{\delta}\left(\mathrm{k}\left(h_{0}\right) \cup \mathrm{k}\left(h_{1}\right)\right)=\mathcal{H}_{\delta}(\mathrm{k}(h))$, since $\mathrm{k}\left(\iota_{0}\right) \subseteq \mathrm{k}\left(\operatorname{tp}\left(h_{1}\right)\right) \stackrel{\mathrm{IH}(\mathrm{e})}{\subseteq} \mathcal{H}_{\delta}\left(\mathrm{k}\left(h_{1}\right)\right)$.

In the same way we get $\mathbf{k}(h[1]) \subseteq \mathcal{H}_{\delta}(\mathbf{k}(h))$. 
2. $h=\mathrm{E}_{\rho^{\prime}}^{\sigma} h_{0}$ : Then $\rho^{\prime} \leq \rho$, and w.l.o.g. we may assume $\rho^{\prime}=\rho$.

So we have $\rho \leq \sigma,\left[\rho, \sigma\left[\cap \mathrm{R}=\emptyset\right.\right.$, and $h_{0} H_{\sigma}^{\beta} \Gamma$ with $\alpha=\widehat{\varphi}_{\sigma\lrcorner \rho}(\beta)$.

2.1. $\operatorname{tp}\left(h_{0}\right)=\mathrm{Cut}_{C}$ with $\rho \leq \nu:=\operatorname{rk}(C)<\sigma$ : Then $\mathcal{I}=\operatorname{Rep}$ and $h[0]=\mathrm{E}_{\rho}^{\nu} \mathrm{R}_{C} \mathrm{E}_{\nu}^{\sigma} h_{0}[0] \mathrm{E}_{\nu}^{\sigma} h_{0}[1]$.

(a) $\Delta(\mathcal{I})=\emptyset$. (c), (d) trivial. (e) $k(\mathcal{I})=\emptyset$.

(b) Let $C^{(0)}:=C, C^{(1)}:=\neg C$. IH $\Longrightarrow h_{0}[i] H_{\sigma}^{\beta_{i}} \Gamma, C^{(i)}$ with $\beta_{i}<\beta \quad(i=0,1) \Longrightarrow$ $\mathrm{E}_{\nu}^{\sigma} h_{0}[i] H_{\nu}^{\widehat{\varphi}_{\nu-\rho}\left(\beta_{i}\right)} \Gamma, C^{(i)}(i=0,1) \Longrightarrow \mathrm{R}_{C} \mathrm{E}_{\nu}^{\sigma} h_{0}[0] \mathrm{E}_{\nu}^{\sigma} h_{0}[1] H_{\nu}^{u} \Gamma \Longrightarrow h[0] H_{\rho}^{\hat{\varphi}_{\nu-\rho}(\eta)} \Gamma$ with $\eta:=\widehat{\varphi}_{\sigma-\nu}\left(\beta_{0}\right) \# \widehat{\varphi}_{\sigma-\nu}\left(\beta_{1}\right)$ and $\left.\widehat{\varphi}_{\nu-\rho}(\eta)<\widehat{\varphi}_{\nu-\rho}\left(\widehat{\varphi}_{\sigma-\nu}(\beta)\right)\right)=\widehat{\varphi}_{\sigma-\rho}(\beta)=\alpha$.

(f) $\mathrm{k}(h[0])=\mathrm{k}\left(h_{0}[0]\right) \cup \mathrm{k}\left(h_{0}[1]\right) \cup\{\nu, \sigma\} \stackrel{\mathrm{IH}}{\subseteq} \mathcal{H}_{\delta}\left(\mathrm{k}\left(h_{0}\right) \cup\{\sigma\}\right) \cup\{\nu\}=\mathcal{H}_{\delta}(\mathrm{k}(h)) \cup\{\nu\}$.

By $\mathrm{IH}(\mathrm{e})$ we have $\mathrm{k}(C)=\mathrm{k}\left(\operatorname{tp}\left(h_{0}\right)\right) \subseteq \mathcal{H}_{\delta}\left(\mathrm{k}\left(h_{0}\right)\right)$ and therefore $\nu=\operatorname{rk}(C) \in \mathcal{H}_{\delta}\left(\mathrm{k}\left(h_{0}\right)\right)$.

2.2. otherwise: $\mathcal{I}=\operatorname{tp}(h)=\operatorname{tp}\left(h_{0}\right), h[\iota]=\mathrm{E}_{\rho}^{\sigma} h_{0}[\iota]$.

(a) $\Delta(\mathcal{I})=\Delta\left(\operatorname{tp}\left(h_{0}\right)\right) \stackrel{\text { IH }}{\subseteq} \Gamma$.

(b) $\mathrm{IH} \Longrightarrow h_{0}[\iota] \mathbb{H}_{\sigma}^{\beta_{\iota}} \Gamma, \Delta_{\iota}(\mathcal{I})$ with $\beta_{\iota}<\beta \Longrightarrow \mathrm{E}_{\rho}^{\sigma} h_{0}[\iota] \mathbb{H}_{\rho}^{\widehat{\varphi}_{\sigma \dot{-} \rho}\left(\beta_{\iota}\right)} \Gamma, \Delta_{\iota}(\mathcal{I})$ with $\widehat{\varphi}_{\sigma-\rho}\left(\beta_{\iota}\right)<\widehat{\varphi}_{\sigma-\rho}(\beta)=\alpha$.

(c) $\mathcal{I}=\mathrm{Cut}_{C} \Rightarrow \operatorname{tp}\left(h_{0}\right)=\mathrm{Cut}_{C} \& \operatorname{rk}(C) \notin[\rho, \sigma[\stackrel{\mathrm{IH}}{\Rightarrow} \operatorname{rk}(C)<\sigma \& \operatorname{rk}(C) \notin[\rho, \sigma[\Rightarrow \operatorname{rk}(C)<\rho$.

(d) $\mathcal{I}=\bigvee_{A}^{\iota_{0}} \Rightarrow \operatorname{tp}\left(h_{0}\right)=\bigvee_{A}^{\iota_{0}} \stackrel{\mathrm{IH}}{\Rightarrow} \mathrm{k}\left(\iota_{0}\right)<\beta \leq \alpha$.

(e) $\mathrm{k}(\mathcal{I})=\mathrm{k}\left(\mathrm{tp}\left(h_{0}\right)\right) \stackrel{\mathrm{IH}}{\subseteq} \mathcal{H}_{\delta}\left(\mathrm{k}\left(h_{0}\right)\right) \subseteq \mathcal{H}_{\delta}(\mathrm{k}(h))$.

(f) $\mathrm{k}(h[\iota])=\mathrm{k}\left(h_{0}[\iota]\right) \cup\{\sigma\} \stackrel{\mathrm{IH}}{\subseteq} \mathcal{H}_{\delta}\left(\mathrm{k}\left(h_{0}\right) \cup \mathrm{k}(\iota) \cup\{\sigma\}\right)=\mathcal{H}_{\delta}(\mathrm{k}(h) \cup \mathrm{k}(\iota))$.

3. $h=\mathrm{B}_{C}^{\beta, \kappa} h_{0}$ with $\beta<\kappa \& C \in \Sigma(\kappa) \cup \Pi(\kappa) \&(C \notin \Pi(\kappa) \Rightarrow \beta \leq \alpha)$ : Then $h_{0} \Vdash_{\rho}^{\alpha} \Gamma, C$ and $C^{(\beta, \kappa)} \in \Gamma$.

3.1. $C \notin \Delta\left(\operatorname{tp}\left(h_{0}\right)\right)$ : Then $\mathcal{I}=\operatorname{tp}\left(h_{0}\right)$ and $h[\iota]=\mathrm{B}_{C}^{\beta, \kappa} h_{0}[\iota]$.

(a) $\Delta(\mathcal{I}) \stackrel{\mathrm{IH}}{\subseteq} \Gamma, C \& C \notin \Delta(\mathcal{I}) \Rightarrow \Delta(\mathcal{I}) \subseteq \Gamma$.

(b) $\mathrm{IH} \Longrightarrow h_{0}[\iota] H_{\rho}^{\alpha_{\iota}} \Gamma, C, \Delta_{\iota}(\mathcal{I})$ with $\alpha_{\iota}<\alpha \Longrightarrow h[\iota]=\mathrm{B}_{C}^{\beta, \kappa} h_{0}[\iota] H_{\rho}^{\alpha_{\iota}} \Gamma, \Delta_{\iota}(\mathcal{I})$ with $\alpha_{\iota}<\alpha$.

(c) $\mathcal{I}=\mathrm{Cut}_{A} \Rightarrow \operatorname{tp}\left(h_{0}\right)=\mathrm{Cut}_{A} \stackrel{\text { IH }}{\Rightarrow} \operatorname{rk}(A)<\rho$.

(d) $\mathcal{I}=\bigvee_{A}^{\iota_{0}} \Rightarrow \operatorname{tp}\left(h_{0}\right)=\bigvee_{A}^{\iota_{0}} \stackrel{\text { IH }}{\Rightarrow} \mathrm{k}\left(\iota_{0}\right)<\alpha$.

(e) $\mathrm{k}(\mathcal{I})=\mathrm{k}\left(\mathrm{tp}\left(h_{0}\right)\right) \stackrel{\mathrm{IH}}{\subseteq} \mathcal{H}_{\delta}\left(\mathrm{k}\left(h_{0}\right)\right) \subseteq \mathcal{H}_{\delta}(\mathrm{k}(h))$.

(f) $\mathrm{k}(h[\iota])=\mathrm{k}\left(h_{0}[\iota]\right) \cup\{\beta\} \stackrel{\mathrm{IH}}{\subseteq} \mathcal{H}_{\delta}\left(\mathrm{k}\left(h_{0}\right) \cup \mathrm{k}(\iota) \cup\{\beta\}\right)=\mathcal{H}_{\delta}(\mathrm{k}(h) \cup \mathrm{k}(\iota))$.

3.2. $C \in \Delta\left(\operatorname{tp}\left(h_{0}\right)\right)$ :

(a) $\Delta(\mathcal{I})=\left\{C^{(\beta, \kappa)}\right\} \subseteq \Gamma$. (c) $\mathcal{I} \neq \mathrm{Cut}_{A}$.

3.2.1. $\operatorname{tp}\left(h_{0}\right)=\bigwedge_{C}$ with $C \simeq \bigwedge\left(C_{\iota}\right)_{\iota \in J}$ :

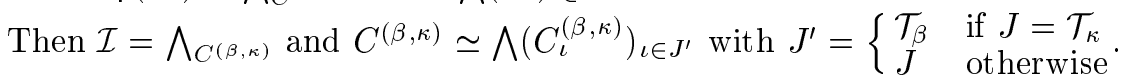

(b) $\mathrm{IH} \Longrightarrow h_{0}[\iota] H_{\rho}^{\alpha_{\iota}} \Gamma, C, C_{\iota}$ with $\alpha_{\iota}<\alpha \Longrightarrow \mathrm{B}_{C}^{(\beta, \kappa)} h_{0}[\iota] H_{\rho}^{\alpha_{\iota}} \Gamma, C_{\iota} \Longrightarrow h[\iota]=\mathrm{B}_{C_{\iota}}^{\beta, \kappa} \mathrm{B}_{C}^{(\beta, \kappa)} h_{0}[\iota] H_{\rho}^{\alpha_{\iota}} \Gamma, C_{\iota}^{(\beta, \kappa)}$, i.e., $h[\iota] H_{\rho}^{\alpha_{\iota}} \Gamma, \Delta_{\iota}(\mathcal{I})$.

(d) $\mathcal{I} \neq \bigvee_{A}^{\iota_{0}}$.

(e) $\mathrm{k}(\mathcal{I})=\mathrm{k}\left(C^{(\beta, \kappa)}\right) \subseteq\{\beta\} \cup \mathrm{k}(C)=\{\beta\} \cup \mathrm{k}\left(\operatorname{tp}\left(h_{0}\right)\right) \stackrel{\mathrm{IH}}{\subseteq} \mathcal{H}_{\delta}\left(\{\beta\} \cup \mathrm{k}\left(h_{0}\right)\right)=\mathcal{H}_{\delta}(\mathrm{k}(h))$.

(f) $\mathrm{k}(h[\iota])=\mathrm{k}\left(h_{0}[\iota]\right) \cup\{\beta\} \stackrel{\mathrm{IH}}{\subseteq} \mathcal{H}_{\delta}\left(\mathrm{k}\left(h_{0}\right) \cup\{\beta\} \cup \mathrm{k}(\iota)\right)=\mathcal{H}_{\delta}(\mathrm{k}(h) \cup \mathrm{k}(\iota))$. 
3.2.2. $\operatorname{tp}\left(h_{0}\right)=\bigvee_{C}^{\iota 0}$ with $C \simeq \bigvee\left(C_{\iota}\right)_{\iota \in J}$ :

Then $\mathcal{I}=\bigvee_{C^{(\beta, \kappa)}}^{\iota_{0}}$ and $C^{(\beta, \kappa)} \simeq \bigvee\left(C_{\iota}^{(\beta, \kappa)}\right)_{\iota \in J^{\prime}}$ with $J^{\prime}=\left\{\begin{array}{ll}\mathcal{T}_{\beta} & \text { if } J=\mathcal{T}_{\kappa} \\ J & \text { otherwise }\end{array}\right.$.

First of all we have to show that $\iota_{0} \in \mathcal{T}_{\beta}$ if $J=\mathcal{T}_{\kappa}$ :

$\operatorname{tp}\left(h_{0}\right)=\bigvee_{C}^{\iota_{0}} \& J=\mathcal{T}_{\kappa} \stackrel{\mathrm{IH}(\mathrm{d})}{\Rightarrow} \mathrm{k}\left(\iota_{0}\right)<\alpha \& C \notin \Pi(\kappa) \Rightarrow \mathrm{k}\left(\iota_{0}\right)<\alpha \leq \beta \Rightarrow \iota_{0} \in \mathcal{T}_{\beta}$.

(b) cf. 3.2.1.

(d) $\operatorname{tp}\left(h_{0}\right)=\bigvee_{C}^{\iota_{0}} \stackrel{\mathrm{IH}(\mathrm{d})}{\Rightarrow} \mathrm{k}\left(\iota_{0}\right)<\alpha$.

(e) $\mathrm{k}(\operatorname{tp}(h))=\mathrm{k}\left(C^{(\beta, \kappa)}\right) \cup \mathrm{k}\left(\iota_{0}\right) \subseteq \mathrm{k}\left(\iota_{0}\right) \cup\{\beta\} \cup \mathrm{k}(C)=\{\beta\} \cup \mathrm{k}\left(\operatorname{tp}\left(h_{0}\right)\right) \stackrel{\mathrm{IH}(\mathrm{e})}{\subseteq} \mathcal{H}_{\delta}\left(\{\beta\} \cup \mathrm{k}\left(h_{0}\right)\right)=\mathcal{H}_{\delta}(\mathrm{k}(h))$.

(f) $\mathrm{k}(h[0])=\mathrm{k}\left(h_{0}[0]\right) \cup\{\beta\} \stackrel{\mathrm{IH}}{\subseteq} \mathcal{H}_{\delta}\left(\mathrm{k}\left(h_{0}\right) \cup\{\beta\}\right)=\mathcal{H}_{\delta}(\mathrm{k}(h))$.

3.2.3. $\operatorname{tp}\left(h_{0}\right)=\operatorname{Ref}_{A}^{\kappa}$ with $C \equiv \exists z \in \mathrm{L}_{\kappa} A^{(z, \kappa)}, A \in \Sigma(\kappa), \kappa \in \mathrm{k}(A)$ : Then $C \notin \Pi(\kappa)$ and thus $\beta \leq \alpha$.

By IH $h_{0}[0] H_{\rho}^{\alpha_{0}} \Gamma, C, A$ with $\alpha_{0}<\alpha \leq \beta$. Now $\mathcal{I}=\bigvee_{C^{(\beta, \kappa)}}^{a}$ and $h[0]=\mathrm{B}_{A}^{\alpha_{0}, \kappa} \mathrm{B}_{C}^{\beta, \kappa} h_{0}[0]$ with $a:=\mathrm{L}_{\alpha_{0}}$.

Further $C^{(\beta, \kappa)} \equiv \exists z \in \mathrm{L}_{\beta} A^{(z, \kappa)} \simeq \bigvee\left(A^{(\iota, \kappa)}\right)_{\iota \in \mathcal{T}_{\beta}}$.

Hence $\mathcal{I}=\bigvee_{C^{(\beta, \kappa)}}^{a}$ is indeed an $\operatorname{RS}^{\infty}$-inference, and $\Delta_{0}(\mathcal{I})=\left\{A^{(a, \kappa)}\right\}=\left\{A^{\left(\alpha_{0}, \kappa\right)}\right\}$.

(b) Since $C^{(\beta, \kappa)} \in \Gamma$, we get $\mathrm{B}_{C}^{\beta, \kappa} h_{0}[0] H_{\rho}^{\alpha_{0}} \Gamma, A$, and then $h[0]=\mathrm{B}_{A}^{\alpha_{0}, \kappa} \mathrm{B}_{C}^{\beta, \kappa} h_{0}[0] \vdash_{\rho}^{\alpha_{0}} \Gamma, A^{\left(\alpha_{0}, \kappa\right)}$.

(d) $\operatorname{lev}(a)=\alpha_{0}<\alpha$.

Proposition: $\alpha_{0} \in \mathcal{H}_{\delta}\left(\mathrm{k}\left(h_{0}\right)\right)$. [Proof: $\left.h_{0}[0] H_{\rho}^{\alpha_{0}} \Gamma, C, A \stackrel{\mathrm{L} .5 .1}{\Longrightarrow} \alpha_{0} \in \mathcal{H}_{\delta}\left(\mathrm{k}\left(h_{0}[0]\right)\right) \stackrel{\mathrm{IH}(\mathrm{f})}{\subseteq} \mathcal{H}_{\delta}\left(\mathrm{k}\left(h_{0}\right)\right)\right]$

(e) $\mathrm{k}(\mathcal{I})=\mathrm{k}\left(C^{(\beta, \kappa)}\right) \cup \mathrm{k}(a) \subseteq \mathrm{k}(C) \cup\left\{\beta, \alpha_{0}\right\}=\mathrm{k}\left(\mathrm{tp}\left(h_{0}\right)\right) \cup\left\{\beta, \alpha_{0}\right\} \stackrel{\text { IH+Prop. }}{\subseteq} \mathcal{H}_{\delta}\left(\mathrm{k}\left(h_{0}\right) \cup\{\beta\}\right)=\mathcal{H}_{\delta}(\mathrm{k}(h))$.

(f) $\mathrm{k}(h[0])=\mathrm{k}\left(h_{0}[0]\right) \cup\left\{\alpha_{0}, \beta\right\} \stackrel{\mathrm{IH}(\mathrm{f})+\text { Prop. }}{\subseteq} \mathcal{H}_{\delta}\left(\mathrm{k}\left(h_{0}\right) \cup\{\beta\}\right)=\mathcal{H}_{\delta}(\mathrm{k}(h))$.

4. $h=\operatorname{Col}_{\gamma}^{\mu, \kappa} h_{0}$ and $\alpha=\psi_{\kappa}\left(\gamma+\omega^{\mu+\tilde{\alpha}}\right) \leq \rho$ with $\mathbf{H}_{\gamma} \ni h_{0} \vdash_{\mu}^{\tilde{\alpha}} \Gamma$ and (w.l.o.g.) $\Gamma \subseteq \Sigma(\kappa)$

and $\mu \in \bar{K} \& \mathbf{k}(h)=\mathbf{k}\left(h_{0}\right) \cup\{\mu, \kappa, \gamma\} \subseteq C_{\kappa}^{*}(\gamma+1) \& \gamma+\omega^{\mu+\tilde{\alpha}} \leq \delta$.

4.1. $\operatorname{tp}\left(h_{0}\right)=\mathrm{Cut}_{C}$ with $\kappa \leq \operatorname{rk}(C)<\mu$ and $\operatorname{rk}(C)=\pi \in \mathrm{R}$ :

W.l.o.g.: $C=\exists x \in \mathrm{L}_{\pi} A(x) \in \Sigma(\pi)$

By IH $(\star) \mathbf{H}_{\gamma} \ni h_{0}[0] \vdash_{\mu}^{\alpha_{0}} \Gamma, C$ and $\mathbf{H}_{\gamma} \ni h_{0}[1] \vdash_{\mu}^{\alpha_{1}} \Gamma, \neg C$ with $\alpha_{0}, \alpha_{1}<\tilde{\alpha}$.

By definition $\mathcal{I}=\operatorname{tp}(h)=\operatorname{Rep}$ and $h[0]=\operatorname{Col}_{\gamma_{1}}^{\mu^{\prime}, \kappa} \mathrm{E}_{\mu^{\prime}}^{\sigma} \mathrm{R}_{C^{(\beta, \pi)}}\left(\mathrm{B}_{C}^{\beta, \pi} \operatorname{Col}_{\gamma}^{\mu, \pi} h_{0}[0]\right)\left(\operatorname{Col}_{\gamma_{0}}^{\mu, \pi} \mathrm{B}_{\neg C}^{\beta, \pi} h_{0}[1]\right)$

with $\gamma_{0}:=\gamma+\omega^{\mu+\alpha_{0}}, \gamma_{1}:=\gamma_{0}+\omega^{\mu+\alpha_{1}}, \beta:=\psi_{\pi}\left(\gamma_{0}\right), \sigma:=\psi_{\pi}\left(\gamma_{1}\right)$,

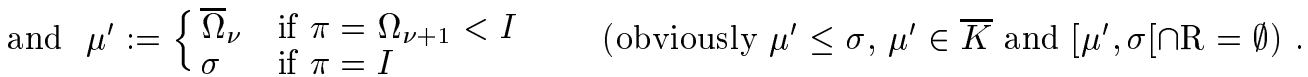

\section{Consequences}

(0) $\forall \gamma^{\prime} \geq \gamma\left[\mathcal{H}_{\gamma^{\prime}}(\mathrm{k}(h)) \subseteq C_{\kappa}^{*}\left(\gamma^{\prime}+1\right)\right]$

(1) $\alpha_{0}, \alpha_{1} \in \mathcal{H}_{\gamma}\left(\mathrm{k}\left(h_{0}\right)\right)$

$\left[h_{0}[i] \in \mathbf{H}_{\gamma} \stackrel{\text { L.5.1 }}{\Rightarrow} \alpha_{i} \in \mathcal{H}_{\gamma}\left(\mathbf{k}\left(h_{0}[i]\right)\right) \stackrel{\mathrm{IH}(\mathrm{f})}{\subseteq} \mathcal{H}_{\gamma}\left(\mathbf{k}\left(h_{0}\right)\right)\right]$

(2) $\gamma_{0}, \gamma_{1} \in \mathcal{H}_{\gamma}(\mathbf{k}(h))$

$\left[\gamma, \mu, \alpha_{0}, \alpha_{1} \in \mathcal{H}_{\gamma}(\mathrm{k}(h))\right]$

(3) $\gamma_{0}, \gamma_{1}<\gamma+\omega^{\mu+\tilde{\alpha}} \leq \delta$

$\left[\alpha_{0}, \alpha_{1}<\tilde{\alpha}\right]$

(4) $\gamma_{0}, \gamma_{1} \in C_{\kappa}^{*}(\gamma+1) \subseteq C_{\pi}\left(\gamma_{i}\right)$

$\left[\alpha_{0}, \alpha_{1} \in \mathcal{H}_{\gamma}\left(\mathrm{k}\left(h_{0}\right)\right) \subseteq C_{\kappa}^{*}(\gamma+1) \& \mu, \gamma \in C_{\kappa}^{*}(\gamma+1)\right]$

(5) $\pi=\operatorname{rk}(C) \in \mathcal{H}_{\gamma}\left(\mathrm{k}\left(h_{0}\right)\right)$

$\left[\mathrm{k}(C)=\mathrm{k}\left(\mathrm{tp}\left(h_{0}\right)\right) \stackrel{\mathrm{IH}(\mathrm{e})}{\subseteq} \mathcal{H}_{\gamma}\left(\mathrm{k}\left(h_{0}\right)\right)\right]$

(6) $\beta \in \mathcal{H}_{\gamma_{0}}(\mathrm{k}(h)) \& \sigma \in \mathcal{H}_{\gamma_{1}}(\mathrm{k}(h))$

$\left[\gamma_{0}, \gamma_{1}, \pi \in \mathcal{H}_{\gamma}(\mathbf{k}(h)) \& \gamma_{i} \stackrel{(4)}{\in} C_{\pi}\left(\gamma_{i}\right)\right]$ 
(7) $\mu^{\prime} \in \mathcal{H}_{\gamma_{1}}(\mathrm{k}(h))$

$\left[\pi, \sigma \in \mathcal{H}_{\gamma_{1}}(\mathrm{k}(h))\right]$

(a) $\Delta(\mathcal{I})=\emptyset$. (c) $\operatorname{tp}(h) \neq \mathrm{Cut}_{A}$. (d) $\operatorname{tp}(h) \neq \bigvee_{A}^{\iota_{0}}$. (e) $\mathrm{k}(\mathcal{I})=\emptyset$

(f) $\mathrm{k}(h[0])=\mathrm{k}\left(h_{0}[0]\right) \cup \mathrm{k}\left(h_{0}[1]\right) \cup\left\{\mu^{\prime}, \kappa, \sigma, \beta, \mu, \pi, \gamma, \gamma_{0}, \gamma_{1}\right\}$.

$\mathrm{k}\left(h_{0}[0]\right) \cup \mathrm{k}\left(h_{0}[1]\right) \cup\{\mu, \kappa, \gamma\} \stackrel{\mathrm{IH}}{\subseteq} \mathcal{H}_{\gamma}\left(\mathrm{k}\left(h_{0}\right) \cup\{\mu, \kappa, \gamma\}\right)=\mathcal{H}_{\gamma}(\mathrm{k}(h))$.

By (1)-(7) we have $\mu^{\prime}, \sigma, \beta, \pi, \gamma_{0}, \gamma_{1} \in \mathcal{H}_{\gamma_{1}}(\mathrm{k}(h))$.

(b) From $(\star)$ we successively obtain

(i) $\mathbf{H}_{\gamma_{0}} \ni d_{0}:=\mathrm{Col}_{\gamma}^{\mu, \pi} h_{0}[0] \vdash_{\beta}^{\beta} \Gamma, C$ and $\mathbf{H}_{\gamma_{0}} \ni d_{1}:=\mathrm{B}_{\neg C}^{\beta, \pi} h_{0}[1] \vdash_{\mu}^{\alpha_{1}} \Gamma, \neg C^{(\beta, \pi)}$.

(ii) $\mathbf{H}_{\gamma_{0}} \ni \mathrm{B}_{C}^{\beta, \pi} d_{0} \vdash_{\beta}^{\beta} \Gamma, C^{(\beta, \pi)}$ and $\mathbf{H}_{\gamma_{1}} \ni \mathrm{Col}_{\gamma_{0}}^{\mu, \pi} d_{1} \vdash_{\sigma}^{\sigma} \Gamma, \neg C^{(\beta, \pi)}$.

(iii) $\mathbf{H}_{\gamma_{1}} \ni e:=\mathrm{R}_{C(\beta, \pi)}\left(\mathrm{B}_{C}^{\beta, \pi} d_{0}\right)\left(\mathrm{Col}_{\gamma_{0}}^{\mu, \pi} d_{1}\right) \vdash_{\sigma}^{\beta \# \sigma} \Gamma$

(iv) $\mathbf{H}_{\gamma_{1}} \ni \mathrm{E}_{\mu^{\prime}}^{\sigma} e \vdash_{\mu^{\prime}}^{\alpha^{*}} \Gamma$ with $\alpha^{*}:=\widehat{\varphi}_{\sigma-\mu^{\prime}}(\beta \# \sigma)$

(v) $\mathbf{H}_{\delta} \ni h[0]=\mathrm{Col}_{\gamma_{1}}^{\mu^{\prime}, \kappa} \mathrm{E}_{\mu^{\prime}}^{\sigma} e \vdash_{\eta}^{\eta} \Gamma$ with $\eta:=\psi_{\kappa}\left(\gamma_{1}+\omega^{\mu^{\prime}+\alpha^{*}}\right)$

ad (i): By IH $\mathrm{k}\left(h_{0}[0]\right) \subseteq \mathrm{k}\left(h_{0}\right)$, and by (5) we have $\pi \in \mathcal{H}_{\gamma}\left(\mathrm{k}\left(h_{0}\right)\right) \subseteq C_{\kappa}^{*}(\gamma+1)$.

Hence $\mathrm{k}\left(h_{0}[0]\right) \cup\{\mu, \pi, \gamma\} \subseteq C_{\kappa}^{*}(\gamma+1)$ and $\Gamma, C \subseteq \Sigma(\pi)$

$\gamma \leq \gamma_{0} \& \beta<\pi \& \neg C \equiv \forall x \in \mathrm{L}_{\pi} \neg A \& \mathrm{k}(A)<\pi$.

ad (ii): $\mathrm{k}\left(d_{1}\right)=\{\beta\} \cup \mathrm{k}\left(h_{0}[1]\right) \stackrel{\mathrm{IH}}{\subseteq}\{\beta\} \cup \mathcal{H}_{\gamma}\left(\mathrm{k}\left(h_{0}\right)\right) \stackrel{(6)}{\subseteq} \mathcal{H}_{\gamma_{0}}(\mathrm{k}(h)) \subseteq C_{\kappa}^{*}\left(\gamma_{0}+1\right) \subseteq C_{\pi}^{*}\left(\gamma_{0}+1\right)$

Now $\mathrm{k}\left(d_{1}\right) \cup\left\{\mu, \pi, \gamma_{0}\right\} \stackrel{(2),(5)}{\subseteq} C_{\pi}^{*}\left(\gamma_{0}+1\right) \& \Gamma, \neg C^{(\beta, \pi)} \subseteq \Sigma(\pi) \& \sigma=\psi_{\pi}\left(\gamma_{1}\right)$ with $\gamma_{1}=\gamma_{0}+\omega^{\mu+\alpha_{1}}$.

ad (iii): $\beta \leq \sigma$ and $\operatorname{rk}\left(C^{(\beta, \pi)}\right)<\sigma$, since $\mathrm{k}\left(C^{(\beta, \pi)}\right)=\{\beta\} \cup(\mathrm{k}(C) \backslash\{\pi\}) \subseteq C_{\pi}\left(\gamma_{1}\right) \cap \pi=\psi_{\pi}\left(\gamma_{1}\right)=\sigma$.

Further $\operatorname{rk}\left(C^{(\beta, \pi)}\right) \notin \mathrm{R}$, since $\beta \notin \mathrm{R}$.

ad (iv): $\mu^{\prime} \leq \sigma$ and $\left[\mu^{\prime}, \sigma[\cap \mathrm{R}=\emptyset\right.$.

ad $(\mathrm{v}): \mathrm{k}\left(\mathrm{E}_{\mu^{\prime}}^{\sigma} e\right) \cup\left\{\mu^{\prime}, \kappa, \gamma_{1}\right\} \subseteq \mathrm{k}(h[0]) \stackrel{(\mathrm{f})}{\subseteq} \mathcal{H}_{\gamma_{1}}(\mathrm{k}(h)) \subseteq C_{\kappa}^{*}\left(\gamma_{1}+1\right)$.

$\gamma_{1}+\omega^{\mu^{\prime}+\alpha^{*}}<\gamma_{1}+\omega^{\mu}<\delta$, since $\mu^{\prime} \leq \sigma<\pi<\mu \& \alpha^{*}<\sigma$.

It remains to prove: $\eta<\alpha$.

For this it suffices to prove: $\gamma_{1}+\omega^{\mu^{\prime}+\alpha^{*}}<\gamma+\omega^{\mu+\tilde{\alpha}}$ and $\gamma_{1}+\omega^{\mu^{\prime}+\alpha^{*}} \in C_{\kappa}\left(\gamma_{1}+\omega^{\mu^{\prime}+\alpha^{*}}\right)$.

The first follows from $\omega^{\mu^{\prime}+\alpha^{*}}<\omega^{\mu}$ and $\gamma_{1}=\gamma+\omega^{\mu+\alpha_{0}}+\omega^{\mu+\alpha_{1}}$ with $\alpha_{0}, \alpha_{1}<\tilde{\alpha}$.

The second is obtained as follows:

$\mathrm{k}(h) \subseteq C_{\kappa}(\gamma+1) \subseteq C_{\kappa}\left(\gamma_{1}+1\right) \Rightarrow \mathcal{H}_{\gamma_{1}}(\mathrm{k}(h)) \subseteq C_{\kappa}\left(\gamma_{1}+1\right) \stackrel{(2),(6),(7)}{\Rightarrow} \gamma_{1}, \alpha^{*}, \mu^{\prime} \in C_{\kappa}\left(\gamma_{1}+1\right)$.

4.2. $\operatorname{tp}\left(h_{0}\right)=$ Cut $_{C}$ with $\kappa \leq \operatorname{rk}(C)<\mu$ and $\operatorname{rk}(C) \notin \mathrm{R}$ :

Let $\pi:=\operatorname{rk}(C)^{+}$. Since $\operatorname{rk}(C)<\mu \leq I+1$ and $\operatorname{rk}(C) \neq I$, we have $\pi<I$ and thus $\pi=\Omega_{\nu+1}$ for some $\nu$.

$\alpha_{i}:=\mathrm{o}\left(h_{0}[i]\right), \gamma_{0}:=\gamma+\omega^{\mu+\alpha_{0}}, \gamma_{1}:=\gamma+\omega^{\mu+\alpha_{1}}, \sigma:=\max \left\{\psi_{\pi}\left(\gamma_{0}\right), \psi_{\pi}\left(\gamma_{1}\right)\right\}, \mu^{\prime}:=\bar{\Omega}_{\nu}, \gamma^{\prime}:=\max \left\{\gamma_{0}, \gamma_{1}\right\}$.

$\operatorname{tp}(h)=\operatorname{Rep}$ and $h[0]=\mathrm{Col}_{\gamma^{\prime}}^{\mu^{\prime}, \kappa} \mathrm{E}_{\mu^{\prime}}^{\sigma} \mathrm{R}_{C}\left(\mathrm{Col}_{\gamma}^{\mu, \pi} h_{0}[0]\right)\left(\mathrm{Col}_{\gamma}^{\mu, \pi} h_{0}[1]\right)$

Proof of (a)-(f) is similar as in case 4.1. 
4.3. Otherwise: Then $\operatorname{tp}\left(h_{0}\right)=\bigwedge_{A}$ or $\bigvee_{A}^{\iota_{0}}$ or $\operatorname{Ref}_{A}^{\tau}$ or $\operatorname{Cut}_{C}$ with $\operatorname{rk}(C) \notin[\kappa, \mu[$.

In each case $\mathcal{I}=\operatorname{tp}\left(h_{0}\right)$ and $h[\iota]=\operatorname{Col}_{\gamma}^{\mu, \kappa} h_{0}[\iota]$.

HS 1: $\forall \iota \in|\mathcal{I}|\left(\mathrm{k}(\iota) \subseteq C_{\kappa}^{*}(\gamma+1)\right)$.

Proof: Let $\mathcal{I}=\bigwedge_{A}$. (Otherwise $|\mathcal{I}| \subseteq\{0,1\}$.) Since $A \stackrel{\mathrm{IH}(\mathrm{a})}{\in} \Gamma \subseteq \Sigma(\kappa)$, there is a $\beta \in \mathrm{k}(A) \cap \kappa$ such that $\forall \iota \in|\mathcal{I}|(\operatorname{lev}(\iota) \leq \beta)$. Since $\mathrm{k}(A)=\mathrm{k}\left(\operatorname{tp}\left(h_{0}\right)\right) \stackrel{\mathrm{IH}(\mathrm{e})}{\subseteq} \mathcal{H}_{\gamma}\left(\mathrm{k}\left(h_{0}\right)\right)$, it follows that $\beta \in C_{\kappa}^{*}(\gamma+1) \cap \kappa$. Hence $\forall \iota \in|\mathcal{I}|\left(\mathrm{k}(\iota) \subseteq C_{\kappa}^{*}(\gamma+1)\right)$, since $C_{\kappa}^{*}(\gamma+1) \cap \kappa$ is an ordinal.

(a) $\Delta(\mathcal{I})=\Delta\left(\operatorname{tp}\left(h_{0}\right)\right) \stackrel{\mathrm{IH}}{\subseteq} \Gamma$.

(b) By IH $\mathbf{H}_{\gamma} \ni h_{0}[\iota] \vdash_{\mu}^{\alpha_{\iota}} \Gamma, \Delta_{\iota}(\mathcal{I})$ with $\alpha_{\iota}<\tilde{\alpha} \quad(\iota \in|\mathcal{I}|)$. (Note that $\operatorname{tp}\left(h_{0}\right)=\mathcal{I}$.)

For each $\iota \in|\mathcal{I}|=\left|\operatorname{tp}\left(h_{0}\right)\right|$ we also have $\mathrm{k}\left(h_{0}[\iota]\right) \stackrel{\mathrm{IH}(\mathrm{f})}{\subseteq} \mathcal{H}_{\gamma}\left(\mathrm{k}\left(h_{0}\right) \cup \mathrm{k}(\iota)\right) \stackrel{\operatorname{HS} 1}{\subseteq} C_{\kappa}^{*}(\gamma+1), \Delta_{\iota}(\mathcal{I}) \stackrel{(\mathrm{a}),(+)}{\subseteq} \Sigma(\kappa)$, $\gamma+\omega^{\mu+\alpha_{\iota}}<\gamma+\omega^{\mu+\tilde{\alpha}} \leq \delta$. Hence $\mathbf{H}_{\delta} \ni h[\iota]=\operatorname{Col}_{\gamma}^{\mu, \kappa} h_{0}[\iota] \vdash_{\eta_{\iota}}^{\eta_{\iota}} \Gamma, \Delta_{\iota}(\mathcal{I})$ with $\eta_{\iota}:=\psi_{\kappa}\left(\gamma+\omega^{\mu+\alpha_{\iota}}\right)$.

$(+) \operatorname{tp}\left(h_{0}\right)=\mathrm{Cut}_{C} \stackrel{\mathrm{IH}(\mathrm{c})}{\Rightarrow} \operatorname{rk}(C)<\mu \Rightarrow \operatorname{rk}(C)<\kappa \Rightarrow C, \neg C \in \Sigma(\kappa)$.

It remains to prove $\eta_{\iota}<\alpha$.

We have $\alpha_{\iota} \stackrel{\text { L.5.1 }}{\in} \mathcal{H}_{\gamma}\left(\mathrm{k}\left(h_{0}[\iota]\right)\right) \stackrel{\mathrm{IH}(\mathrm{f})}{\subseteq} \mathcal{H}_{\gamma}\left(\mathrm{k}\left(h_{0}\right) \cup \mathrm{k}(\iota)\right) \stackrel{\text { HS1 }}{\subseteq} C_{\kappa}(\gamma+1)$ and $\mu, \gamma \in C_{\kappa}(\gamma+1)$. Hence $\gamma+\omega^{\mu+\alpha_{\iota}} \in$ $C_{\kappa}\left(\gamma+\omega^{\mu+\alpha_{\iota}}\right)$. Together with $\alpha_{\iota}<\tilde{\alpha}$ this yields $\eta_{\iota}=\psi_{\kappa}\left(\gamma+\omega^{\mu+\alpha_{\iota}}\right)<\psi_{\kappa}\left(\gamma+\omega^{\mu+\tilde{\alpha}}\right)=\alpha$.

(c) Assume $\mathcal{I}=\mathrm{Cut}_{C}$. Then $\operatorname{tp}\left(h_{0}\right)=\mathrm{Cut}_{C}$ with $\operatorname{rk}(C) \notin[\kappa, \mu[$. By $\operatorname{IH}(\mathrm{c})$ that $\operatorname{rk}(C)<\mu$ and thus $\operatorname{rk}(C)<\kappa$. By IH(e) $\mathrm{k}(C)=\mathrm{k}\left(\operatorname{tp}\left(h_{0}\right)\right) \subseteq \mathcal{H}_{\gamma}\left(\mathrm{k}\left(h_{0}\right)\right) \subseteq C_{\kappa}^{*}(\gamma+1)$ and thus

$\operatorname{rk}(C) \in C_{\kappa}^{*}(\gamma+1) \cap \kappa \subseteq C_{\kappa}(\gamma+1) \cap \kappa=\psi_{\kappa}(\gamma+1) \leq \alpha \leq \rho$.

(d) Assume $\mathcal{I}=\bigvee_{A}^{\iota_{0}}$. Then $\operatorname{tp}\left(h_{0}\right)=\bigvee_{A}^{\iota_{0}}$, and by $\operatorname{IH}\left(\right.$ a) we have $A \in \Gamma \subseteq \Sigma(\kappa)$. Hence $\mathrm{k}\left(\iota_{0}\right) \subseteq \kappa$. Further $\mathrm{k}\left(\iota_{0}\right) \subseteq \mathrm{k}\left(\mathrm{tp}\left(h_{0}\right)\right) \stackrel{\mathrm{IH}(\mathrm{e})}{\subseteq} \mathcal{H}_{\gamma}\left(\mathrm{k}\left(h_{0}\right)\right) \subseteq C_{\kappa}(\gamma+1)$. Hence $\mathrm{k}\left(\iota_{0}\right) \subseteq C_{\kappa}(\gamma+1) \cap \kappa=\psi_{\kappa}(\gamma+1) \leq \alpha$.

(e) $\mathrm{k}(\mathcal{I})=\mathrm{k}\left(\operatorname{tp}\left(h_{0}\right)\right) \stackrel{\mathrm{IH}(\mathrm{e})}{\subseteq} \mathcal{H}_{\gamma}\left(\mathrm{k}\left(h_{0}\right)\right) \subseteq \mathcal{H}_{\delta}(\mathrm{k}(h))$.

(f) $\mathrm{k}(h[\iota])=\{\mu, \kappa, \gamma\} \cup \mathrm{k}\left(h_{0}[\iota]\right) \stackrel{\mathrm{IH}}{\subseteq} \mathcal{H}_{\gamma}\left(\{\mu, \kappa, \gamma\} \cup \mathrm{k}\left(h_{0}\right) \cup \mathrm{k}(\iota)\right)=\mathcal{H}_{\gamma}(\mathrm{k}(h) \cup \mathrm{k}(\iota))$.

\section{Theorem 5.3}

If $\mathrm{KPi} \vdash \forall z\left(" z=\mathrm{HF}\right.$ " $\left.\rightarrow \phi^{z}\right)$ with $\mathrm{FV}(\phi)=\emptyset$, then there exists an $h \in \mathbf{H}_{\delta}$ (for some $\delta<\varepsilon_{I+1}$ ) such that $h \vdash_{0}^{\beta} \phi^{L_{\omega}}$ with $\beta<\psi_{\Omega}\left(\varepsilon_{I+1}\right)$. (The formula " $z=\mathrm{HF}$ " is defined on page 8.)

Proof:

Then there is a conjunction $\chi$ of KPi-axioms and an $\mathrm{RS}^{1}$-derivation $d_{1}$ of $\neg\left(\chi^{I} \wedge\right.$ " $z=\mathrm{HF}$ "), $\phi^{z}$ with $\mathrm{o}\left(d_{1}\right)<\omega^{I+\omega}, \operatorname{deg}\left(d_{1}\right)<I+\omega, \mathrm{k}\left(d_{1}\right) \subseteq\{0, I\}$ and $\mathrm{FV}\left(d_{1}\right) \subseteq\{z\}$ (cf. Theorem 3.6).

Further we have an $\mathrm{RS}^{0}$-derivation $d_{0}$ of $\chi^{I} \wedge$ " $\mathrm{L}_{\omega}=\mathrm{HF}$ " with $\mathrm{o}\left(d_{0}\right)<\omega^{I+\omega}, \operatorname{deg}\left(d_{0}\right) \leq I$, and $\mathrm{k}\left(d_{0}\right) \subseteq\{0, \omega, I\}$. ( $d_{0}$ is build up by $\wedge$-inferences from $\bigvee_{\exists x \in \mathrm{L}_{\omega}(x \subseteq x)}^{\mathrm{L}_{0}} \mathrm{Ax}_{4}^{*}\left(\mathrm{~L}_{0} \subseteq \mathrm{L}_{0}\right)$ and atomic $\mathrm{RS}^{0}$-derivations of the following kinds: $\left.\mathrm{Ax}_{1}^{*}\left(\psi^{\lambda}\right)(\lambda \in\{\omega, I\}), \mathrm{Ax}_{2}^{*}\left(\psi^{I}\right), \mathrm{Ax}_{6}^{*}\left(\operatorname{tran}\left(\mathrm{L}_{\omega}\right)\right), \mathrm{Ax}_{15}^{*}\left(\forall x \in \mathrm{L}_{\omega} \exists u \in \mathrm{L}_{\omega}(\exists y \in u(x \in y) \wedge \mathcal{A}(u))\right).\right)$

Let $C: \equiv \chi^{I} \wedge$ "L ${ }_{\omega}=$ HF", and choose $0<m<\omega$ such that $\max \left\{\operatorname{rk}(C), \operatorname{deg}\left(d_{0}\right), \operatorname{deg}\left(d_{1}\right)\right\} \leq I+m$.

(0) $d_{0} \vdash_{I+m}^{\alpha_{0}} \chi^{I} \wedge$ "L ${ }_{\omega}=\mathrm{HF}$ " and $d_{1}\left(z / \mathrm{L}_{\omega}\right) \vdash_{I+m}^{\alpha_{1}} \neg\left(\chi^{I} \wedge\right.$ "L $\left.\mathrm{L}_{\omega}=\mathrm{HF} "\right), \phi^{\mathrm{L}_{\omega}}$ with $\alpha_{0}, \alpha_{1}<\varepsilon_{I+1}$

(1) $h_{0}:=\mathrm{R}_{C} d_{0} d_{1}\left(z / \mathrm{L}_{\omega}\right) \vdash_{I+m}^{\alpha_{0} \# \alpha_{1}} \phi^{\mathrm{L}_{\omega}}$ 
(2) $h^{\prime}:=\mathrm{E}_{I+1}^{I+m} h_{0} \vdash_{I+1}^{\alpha} \phi^{\mathrm{L}_{\omega}}$ with $\alpha:=\widehat{\varphi}_{m-1}\left(\alpha_{0} \# \alpha_{1}\right)=\omega_{m-1}\left(\alpha_{0} \# \alpha_{1}\right)<\varepsilon_{I+1}$

(3) $h^{\prime \prime}:=\operatorname{Col}_{0}^{I+1, \Omega} h^{\prime} \vdash_{\sigma}^{\sigma} \phi^{\mathrm{L}_{\omega}}$ with $\sigma:=\psi_{\Omega}\left(\omega^{I+1+\alpha}\right)<\psi_{\Omega}\left(\varepsilon_{I+1}\right)$

(4) $h:=\mathrm{E}_{0}^{\sigma} h^{\prime \prime} \vdash_{0}^{\beta} \phi^{\mathrm{L}_{\omega}}$ with $\beta:=\widehat{\varphi}_{\sigma}(\sigma)<\psi_{\Omega}\left(\varepsilon_{I+1}\right)$

ad (1): $d_{0}, d_{1}\left(z / \mathrm{L}_{\omega}\right) \in \mathbf{H}_{0} \& \operatorname{rk}(C) \notin \mathrm{R} \Rightarrow h_{0} \in \mathbf{H}_{0}$

ad (2): $h_{0} \in \mathbf{H}_{0} \&\left[I+1, I+m\left[\cap \mathbf{R}=\emptyset \& \operatorname{deg}\left(h_{0}\right) \leq I+m \Rightarrow h^{\prime} \in \mathbf{H}_{0}\right.\right.$

ad (3): $\mathrm{k}\left(h^{\prime}\right)=\{I+m\} \cup \mathrm{k}\left(h_{0}\right)=\{I+m, \omega\} \cup \mathrm{k}\left(d_{0}\right) \cup \mathrm{k}\left(d_{1}\right) \subseteq\{0, \omega, I, I+m\} \subseteq C_{\Omega}^{*}(1)$

$h^{\prime} \in \mathbf{H}_{0} \& I+1 \in \bar{K} \& \mathbf{k}\left(h^{\prime}\right) \cup\{I+1, \Omega, 0\} \subseteq C_{\Omega}^{*}(1) \& \operatorname{End}\left(h^{\prime}\right) \subseteq\left\{\phi^{L_{\omega}}\right\} \subseteq \Sigma(\Omega) \& \operatorname{deg}\left(h^{\prime}\right)=I+1 \Longrightarrow$ $h^{\prime \prime}=\operatorname{Col}_{0}^{I+1, \Omega} h^{\prime} \in \mathbf{H}_{\delta}$ with $\delta:=\omega^{I+1+\mathrm{o}\left(h^{\prime}\right)}$

ad (4): $h^{\prime \prime} \in \mathbf{H}_{\delta} \& \operatorname{deg}\left(h^{\prime \prime}\right) \leq \sigma<\Omega \Rightarrow h \in \mathbf{H}_{\delta}$.

$\S 6$ Bounding provable $\Pi_{2}^{0}$-sentences

\section{Definition}

$2_{0}:=0,2_{m+1}:=2^{2 m}$

$\mathbf{s}_{n}:= \begin{cases}\mathrm{L}_{0} & \text { if } n=0 \\ {\left[x \in \mathrm{L}_{l+1}: x=\mathrm{s}_{n_{0}} \vee \ldots \vee x=\mathrm{s}_{n_{k}}\right]} & \text { if } n=2^{n_{0}}+\ldots+2^{n_{k}} \text { with } n_{0}>\ldots>n_{k} \text { and } l:=\operatorname{lev}\left(\mathbf{s}_{n_{0}}\right)\end{cases}$

\section{Lemma 6.1}

a) $\mathbf{s}_{n}$ is an RS-term with $\operatorname{lev}\left(\mathbf{s}_{n}\right)<\omega$,

b) $\operatorname{lev}\left(\mathbf{s}_{n}\right)<m \Leftrightarrow n<2 m$.

Proof:

a) It suffices to prove: $n \leq m \Rightarrow \operatorname{lev}\left(\mathbf{s}_{n}\right) \leq \operatorname{lev}\left(\mathbf{s}_{m}\right)$.

Induction on $n: 1$. $n=0$ : trivial.

2. $n=2^{n_{0}}+\ldots+2^{n_{k}}$ with $n>n_{0}>\ldots>n_{k}$ : Then $m=2^{m_{0}}+\ldots+2^{m_{l}}$ with $m_{0}>\ldots>m_{l}$ and $n_{0} \leq m_{0}$.

Hence $\operatorname{lev}\left(s_{n}\right)=\operatorname{lev}\left(s_{n_{0}}\right)+1 \stackrel{\mathrm{IH}}{\leq} \operatorname{lev}\left(\mathbf{s}_{m_{0}}\right)+1=\operatorname{lev}\left(\mathbf{s}_{m}\right)$.

b) Let $m>0$, since for $m=0$ the claim is trivial.

1. $n=0: \operatorname{lev}\left(\mathrm{s}_{0}\right)=\operatorname{lev}\left(\mathrm{L}_{0}\right)=0$.

2. $n=2^{n_{0}}+\ldots+2^{n_{k}}: \operatorname{lev}\left(\mathrm{s}_{n}\right)<m \Leftrightarrow \operatorname{lev}\left(\mathrm{s}_{n_{0}}\right)<m-1 \stackrel{\mathrm{IH}}{\Leftrightarrow} n_{0}<2_{m-1} \Leftrightarrow n<2_{m}$

Definition $\mathcal{T}_{m}^{*}:=\left\{\mathbf{s}_{n}: \operatorname{lev}\left(\mathbf{s}_{n}\right)<m\right\}=\left\{\mathbf{s}_{n}: n<2_{m}\right\}$

Definition of $\models A$ by recursion on $\operatorname{rk}(A)$

$=A: \Leftrightarrow \begin{cases}\forall \iota \in J\left(\mid=A_{\iota}\right) & \text { if } A \simeq \bigwedge\left(A_{\iota}\right)_{\iota \in J} \\ \exists \iota \in J\left(\mid=A_{\iota}\right) & \text { if } A \simeq \bigvee\left(A_{\iota}\right)_{\iota \in J}\end{cases}$

Abbreviation: $\mid=\Gamma: \Leftrightarrow \exists A \in \Gamma(\mid=A)$

\section{Lemma 6.2}

a) $=\neg A \Leftrightarrow \not \forall A$

b) If $(\mathcal{D}, \mathrm{o}, \operatorname{deg}, \mathrm{tp},[])$ is a notation system for $\mathrm{RS}^{\infty}$-derivations such that $\forall d \in \mathcal{D}\left(\operatorname{tp}(d) \neq \operatorname{Ref}_{A}^{\kappa}\right)$, 
then $\models \operatorname{End}(d)$ holds for each $d \in \mathcal{D}$.

c) $\quad=s \neq t, \neg A(s), A(t)$

Proof:

a) Induction on $\operatorname{rk}(A)$ :

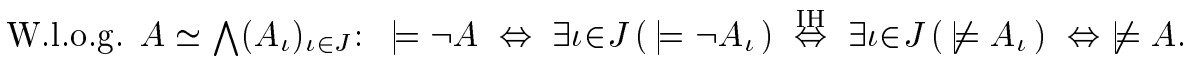

b) Induction on $\mathrm{o}(d)$ : Let $(\mathrm{a}), \ldots,(\mathrm{e})$ refer to the conditions in the definition of "notation system" in $\S 1$.

1. $\operatorname{tp}(d)=\bigwedge_{A}$ with $A \simeq \bigwedge\left(A_{\iota}\right)_{\iota \in J}$ : Then by (b) $\forall \iota \in J\left(\operatorname{End}(d[\iota]) \subseteq \operatorname{End}(d), A_{\iota}\right)$ and therefore by (e) and IH $\forall \iota \in J\left(\models \operatorname{End}(d), A_{\iota}\right)$. Hence $\models \operatorname{End}(d)$, since by (a) $A \in \operatorname{End}(d)$.

2. $\operatorname{tp}(d)=\bigvee_{A}^{\iota_{0}}$ or $\mathrm{Cut}_{C}$ or Rep: analogous to 1 .

c) Let $\mathrm{RS}^{00}$ be the subsystem of $\mathrm{RS}^{0}$ (from $\S 2$ ) consisting of the inferences $A x_{j}^{*}(\Pi)(j \in\{12,13,14\})$ and $\bigwedge_{A_{0} \wedge A_{1}}, \bigvee_{A}^{\iota_{0}}$. One easily verifies that for every $\mathrm{RS}^{00}$-derivation $d$ we have:

$-\operatorname{tp}(d) \neq \operatorname{Ref}_{A}^{\kappa}$ (and also $\operatorname{tp}(d) \neq \operatorname{Cut}_{C}$ and $\operatorname{tp}(d) \neq \operatorname{Rep}$ ),

- for each $\iota \in|\operatorname{tp}(d)|, d[\iota]$ is again an $\operatorname{RS}^{00}$-derivation.

Now by Theorem 2.1 and $\mathrm{b}$ ) above we get $\models \operatorname{End}(d)$ for each $\mathrm{RS}^{00}$-derivation $d$. Hence $\models s \neq t, \neg A(s), A(t)$.

\section{Lemma 6.3}

For every $a \in \mathcal{T}_{\omega}$ there is an $n<\omega$ such that $=a=\mathrm{s}_{n}$ and $\operatorname{lev}\left(\mathrm{s}_{n}\right) \leq \operatorname{lev}(a)$.

Proof by induction on $\operatorname{lev}(a)$ : Let $a \equiv\left[x \in \mathrm{L}_{m}: A(x)\right]$.

I. Assume $\emptyset \neq\left\{l<2_{m}: \models A\left(s_{l}\right)\right\}$. Let $n_{0}>\ldots>n_{k}$ such that $\left\{n_{0}, \ldots, n_{k}\right\}=\left\{l<2_{m}: \mid=A\left(s_{l}\right)\right\}$

Let $n:=2^{n_{0}}+\ldots+2^{n_{k}}$. Then $\mathbf{s}_{n} \equiv\left[x \in \mathrm{L}_{m_{1}}: x=\mathbf{s}_{n_{0}} \vee \ldots \vee x=\mathbf{s}_{n_{k}}\right]$.

$n_{0}<2_{m} \Rightarrow n<2_{m+1} \stackrel{6.1 \mathrm{~b}}{\Rightarrow} m_{1}=\operatorname{lev}\left(\mathbf{s}_{n}\right) \leq m=\operatorname{lev}(a)$.

Abb.: $s_{i}:=\mathbf{s}_{n_{i}}$

" $\models \mathrm{s}_{n} \subseteq a$ ":

Note that $s_{n} \subseteq a \simeq \bigwedge\left(t=s_{0} \vee \ldots \vee t=s_{k} \rightarrow t \in a\right)_{t \in \mathcal{T}_{m_{1}}}$ and $t \in a \simeq \bigvee(A(s) \wedge s=t)_{s \in \mathcal{T}_{m}}$.

$=A\left(s_{i}\right)(i \in\{0, \ldots, k\}) \Rightarrow \models t \neq s_{i}, A\left(s_{i}\right) \wedge s_{i}=t\left(i \in\{0, \ldots, k\}, t \in \mathcal{T}_{m_{1}}\right) \Rightarrow$

$=t \neq s_{i}, t \in a\left(i \in\{0, \ldots, k\}, t \in \mathcal{T}_{m_{1}}\right) \Rightarrow \models t=s_{0} \vee \ldots \vee t=s_{k} \rightarrow t \in a\left(t \in \mathcal{T}_{m_{1}}\right) \Rightarrow \mid=\mathrm{s}_{n} \subseteq a$.

" $\models a \subseteq \mathrm{s}_{n}$ ":

Note that $a \subseteq s_{n} \simeq \bigwedge\left(A(t) \rightarrow t \in \mathrm{s}_{n}\right)_{t \in \mathcal{T}_{m}}$ and $t \in \mathrm{s}_{n} \simeq \bigvee\left(\left(s=s_{0} \vee \ldots \vee s=s_{k}\right) \wedge s=t\right)_{s \in \mathcal{T}_{m_{1}}}$

Let $t \in \mathcal{T}_{m}$ such that $\models A(t)$. We have to prove $\models t \in \mathbf{s}_{n}$.

By IH there is an $l$ such that $\models t=s_{l}$ and $\operatorname{lev}\left(s_{l}\right) \leq \operatorname{lev}(t)<m$, i.e. $l<2_{m}$.

By Lemma 6.2 and the assumption $\mid=A(t)$ we get $\mid=A\left(s_{l}\right)$ and thus $l \in\left\{n_{0}, \ldots, n_{k}\right\}$. So we have $\mid=t=s_{i}$ for some $i \in\{0, \ldots, k\}$. This yields $\models\left(s_{i}=s_{0} \vee \ldots \vee s_{i}=s_{k}\right) \wedge s_{i}=t$ and then $\models t \in \mathbf{s}_{n}$, since $s_{i} \in \mathcal{T}_{m_{1}}$.

II. Assume $\left\{l<2_{m}: \models A\left(s_{l}\right)\right\}=\emptyset$ : Then we prove $\mid=a=\mathrm{L}_{0}$.

" $\models \mathrm{L}_{0} \subseteq a$ ": trivial.

" $\models a \subseteq \mathrm{L}_{0}$ ": We have $a \subseteq \mathrm{L}_{0} \simeq \bigwedge\left(A(t) \rightarrow t \in \mathrm{L}_{0}\right)_{t \in \mathcal{T}_{m}}$ and $t \in \mathrm{L}_{0} \simeq \bigvee(s=t)_{s \in \mathcal{T}_{0}}$. 
Let $t \in \mathcal{T}_{m}$. By IH there is an $l$ such that $\models t=\mathrm{s}_{l}$ and $\operatorname{lev}\left(\mathrm{s}_{l}\right) \leq \operatorname{lev}(t)<m$, i.e. $l<2_{m}$. Then $\models \neg A\left(\mathrm{~s}_{l}\right)$, and by Lemma 6.2 we obtain $\models \neg A(t)$ and then $\models A(t) \rightarrow t \in \mathrm{L}_{0}$.

\section{Lemma 6.4}

If $A \simeq \bigwedge\left(A_{\iota}\right)_{\iota \in \mathcal{T}_{m}}$ and $\models A_{\iota}$ for all $\iota \in \mathcal{T}_{m}^{*}$, then $=A$.

Proof: There is a formula $C(x)$ such that $A_{t}: \equiv C(t)$ for all $t \in \mathcal{T}_{m}$. We have to prove $\models C(t)$ for all $t \in \mathcal{T}_{m}$. Let $t \in \mathcal{T}_{m}$. By 6.3 there is a $t^{*} \in \mathcal{T}_{m}^{*}$ with $\models t=t^{*}$. By assumption we have $\models C\left(t^{*}\right)$. Hence $\models C(t)$ by Lemma $6.2 \mathrm{c}$.

Definition of $f(h, n)$ for $h \in \mathbf{H}_{\delta}$ and $n \in \omega$

For $A \simeq \Lambda\left(A_{\iota}\right)_{\iota \in J}$ we set $|A|^{n}:=\left\{\begin{array}{ll}\mathcal{T}_{m}^{*} & \text { if } J=\mathcal{T}_{m} \\ \mathcal{T}_{n}^{*} & \text { if } J=\mathcal{T}_{\omega} \\ J & \text { if } J=\{0,1\} \\ \emptyset & \text { otherwise }\end{array}\right.$.

$f(h, n):= \begin{cases}f(h[0], n) & \text { if } \operatorname{tp}(h)=\operatorname{Rep} \\ \max \left\{f(h[0], n), \operatorname{lev}\left(\iota_{0}\right)+1\right\} & \text { if } \operatorname{tp}(h)=\bigvee_{A}^{\iota_{0}} \text { and } \operatorname{lev}\left(\iota_{0}\right)<\omega \\ \max \left\{f(h[\iota], n): \iota \in|A|^{n}\right\} & \text { if } \operatorname{tp}(h)=\bigwedge_{A} \\ 0 & \text { otherwise }\end{cases}$

Here we understand that $\max (\emptyset)=0$.

\section{Definition}

$A^{n, k}$ results from $A$ by replacing every $\forall x \in \mathrm{L}_{\omega}$ by $\forall x \in \mathrm{L}_{n}$, and every $\exists x \in \mathrm{L}_{\omega}$ by $\exists x \in \mathrm{L}_{k}$.

$\Gamma^{n, k}:=\left\{A^{n, k}: A \in \Gamma\right\}$

$\mathrm{RS}_{\omega}:=$ set of all $\mathrm{RS}_{\omega}$-sentences (cf. $\S 3$ )

\section{Lemma 6.5}

$h \in \mathbf{H}_{\delta} \& \operatorname{End}(h) \subseteq \operatorname{RS}_{\omega} \& \operatorname{deg}(h)=0 \& f(h, n) \leq k \Longrightarrow \mid=\operatorname{End}(h)^{n, k}$.

Proof:

Let $h \in \mathbf{H}_{\delta} \& \operatorname{End}(h) \subseteq \operatorname{RS}_{\omega} \& \operatorname{deg}(h)=0 \& f(h, n) \leq k$.

Then for each $\iota \in|\operatorname{tp}(h)|$ we have $h[\iota] \in \mathbf{H}_{\delta} \& \operatorname{End}(h[\iota]) \subseteq \operatorname{RS}_{\omega} \& \operatorname{deg}(h[\iota])=0 \& \mathrm{o}(h[\iota])<\mathrm{o}(h)$.

Hence the IH applies to each $h[\iota](\iota \in|\operatorname{tp}(h)|)$.

Again by Theorem 5.2 one of the following cases holds:

$\operatorname{tp}(h)=\bigvee_{A}^{\iota_{0}}$ with $\operatorname{lev}\left(\iota_{0}\right)<\omega, \operatorname{tp}(h)=\bigwedge_{A}$ with $|\operatorname{tp}(h)|=\mathcal{T}_{\alpha}$ and $\alpha \leq \omega, \operatorname{tp}(h)=\bigwedge_{A_{0} \wedge A_{1}}, \operatorname{tp}(h)=\operatorname{Rep}$.

We only treat the first two cases. - Abbreviation: $\Gamma:=\operatorname{End}(h)$.

1. $\operatorname{tp}(h)=\bigvee_{A}^{\iota_{0}}$ with $A \simeq \bigvee\left(A_{\iota}\right)_{\iota \in J}$ and $\operatorname{lev}\left(\iota_{0}\right)<\omega$ : Then $k \geq \max \left\{f(h[0], n), \operatorname{lev}\left(\iota_{0}\right)+1\right\}$

By $5.2 \mathrm{~b} \operatorname{End}(h[0]) \subseteq \Gamma, A_{\iota_{0}}$, and therefore by $\mathrm{IH} \models \Gamma^{n, k}, A_{\iota_{0}}^{n, k}$. This implies $\models \Gamma^{n, k}$, since $A \in \Gamma$ (by 5.2a) and $A^{n, k} \simeq \bigvee\left(A_{\iota}^{n, k}\right)_{\iota \in J^{\prime}}$ with $\iota_{0} \in J^{\prime}$. The latter follows from $\operatorname{lev}\left(\iota_{0}\right)<k \& \iota_{0} \in J$.

2.1. $\operatorname{tp}(h)=\bigwedge_{A}$ with $A \simeq \bigwedge\left(A_{t}\right)_{t \in \mathcal{T}_{m}}$ : Then $f(h[t], n) \leq k$ for all $t \in \mathcal{T}_{m}^{*}$.

By $5.2 \mathrm{~b}$ and IH $\mid=\Gamma^{n, k}, A_{t}^{n, k}$ for all $t \in \mathcal{T}_{m}^{*}$.

Since $A \in \Gamma$ and $A^{n, k} \simeq \bigwedge\left(A_{t}^{n, k}\right)_{t \in \mathcal{T}_{m}}$, this yields $\models \Gamma^{n, k}$ by Lemma 6.4. 


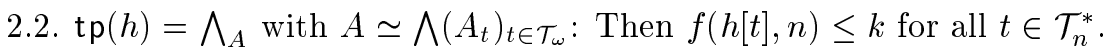

By $5.2 \mathrm{~b}$ and $\mathrm{IH}=\Gamma^{n, k}, A_{t}^{n, k}$ for all $t \in \mathcal{T}_{n}^{*}$.

Since $A \in \Gamma$ and $A^{n, k} \simeq \bigwedge\left(A_{t}\right)_{t \in \mathcal{T}_{n}}$ this yields $\models \Gamma^{n, k}, A^{n, k}$ by Lemma 6.4.

\section{Theorem 6.6}

If KPi $\vdash \forall z$ ("z=HF" $\rightarrow \forall x \in z \exists y \in z \phi(x, y))$ with $\phi(x, y) \in \Delta_{0}$, then there exists an $h \in \mathbf{H}_{\delta}$ (for some $\left.\delta<\varepsilon_{I+1}\right)$ such that $\mathrm{o}(h)<\psi_{\Omega}\left(\varepsilon_{I+1}\right)$ and $\models \forall x \in \mathrm{L}_{n} \exists y \in \mathrm{L}_{f(h, n)} \phi(x, y)$ for all $n \in \omega$.

Proof:

By Theorem 5.3 we have an $h \in \mathbf{H}_{\delta}$ with o $(h)<\psi_{\Omega}\left(\varepsilon_{I+1}\right)$, $\operatorname{End}(h)=\left\{\forall x \in \mathbf{L}_{\omega} \exists y \in \mathbf{L}_{\omega} \phi(x, y)\right\}$, and $\operatorname{deg}(h)=0$. By Lemma 6.5 this yields $\models \forall x \in \mathrm{L}_{n} \exists y \in \mathrm{L}_{f(h, n)} \phi(x, y)$.

\section{Remark}

Assuming a canonical arithmetization of ordinals $\alpha \in C_{\Omega}\left(\varepsilon_{I+1}\right)$ and of finitary derivations (notations) $h \in \mathbf{H}_{\delta}$ the function $f$ is $\prec$-primitive recursive in the sense of [Tak87], p.117, where $\prec$ is a primitive recursive wellordering of ordertype $\psi_{\Omega}\left(\varepsilon_{I+1}\right)$. For fixed $h$ as in 6.6 the function $n \mapsto f(h, n)$ is $\prec \mid \alpha$-primitive recursive with $\alpha:=\mathrm{o}(h)<\psi_{\Omega}\left(\varepsilon_{I+1}\right)$.

\section{References}

[Ar96] Arai, T.: Proof Theory for Theories of Ordinals I: Reflecting Ordinals. Preprint (1996)

[Ar97a] Arai, T.: Proof Theory for Theories of Ordinals II: $\Sigma_{1}$-stability. Preprint (1997)

[Ar97b] Arai, T.: Proof Theory for Theories of Ordinals III: $\Pi_{1}$-collection. Preprint (1997)

[Bla97] Blankertz, B.: Beweistheoretische Techniken zur Bestimmung von $\Pi_{2}^{0}$-Skolem Funktionen. Dissertation, Münster 1997.

[Bu86] Buchholz, W.: A new system of proof-theoretic ordinal functions. APAL 32, pp. 195-207 (1986)

[Bu91] Buchholz, W.: Notation systems for infinitary derivations. Arch. Math. Logic 30, pp. 277-296 (1991)

[Bu92] Buchholz, W.: A simplified version of local predicativity. In: Proof Theory, Leeds 1990. Aczel, Simmons, Wainer (eds.) Cambridge University Press (1992), 115-147.

[Bu97] Buchholz, W.: Explaining the Gentzen-Takeuti reduction steps. Preprint (1997)

[Jä84] Jäger, G.: $\rho$-inaccessible ordinals, collapsing functions and a recursive notation system. Arch. math. Logik 24 (1984), pp. 49-62.

[Ra91] Rathjen, M.: Proof Theoretic Analysis of KPM. Arch. Math. Logic 30 (1991) pp. 377-403

[Ra94] Rathjen, M.: Proof Theory of Reflection. APAL 68 (1994) pp. 181-224

[Ra95] Rathjen, M.: Recent Advances in Ordinal Analysis: $\Pi_{2}^{1}-C A$ and related systems. The Bulletin of Symbolic Logic 1/4 (1995) pp. 468-485

[Tak87] Takeuti, G.: Proof Theory. 2nd edition. Amsterdam: North-Holland 1987

[Tu98] Tupailo, S.: Finitary reductions for local predicativity. Preprint (1998) 